\title{
Automotive Intelligence Embedded in Electric Connected Autonomous and Shared Vehicles Technology for Sustainable Green Mobility
}

\author{
Ovidiu Vermesan ${ }^{1 *}$, Reiner John ${ }^{2}$, Patrick Pype ${ }^{3}$, Gerardo Daalderop ${ }^{3}$, Kai Kriegel ${ }^{4}$, \\ Gerhard Mitic $^{4}$, Vincent Lorentz ${ }^{5}$, Roy Bahr ${ }^{1}$, Hans Erik Sand ${ }^{6}$, Steffen Bockrath ${ }^{5}$ and \\ Stefan Waldhör ${ }^{5}$
}

${ }^{1}$ SINTEF Digital AS, Oslo, Norway, ${ }^{2}$ AVL List GmbH, Graz, Austria, ${ }^{3}$ NXP Semiconductors, Leuven, Belgium, ${ }^{4}$ Siemens AG, Munich, Germany, ${ }^{5}$ Fraunhofer IISB, Erlangen, Germany, ${ }^{6}$ NxTech AS, Fredrikstad, Norway

\section{OPEN ACCESS}

Edited by:

Emmanouil Rigas,

University of Cyprus, Cyprus

Reviewed by:

Andreas Seitaridis,

Centre for Research and Technology Hellas (CERTH), Greece Giuseppe Avino,

Politecnico di Torino, Italy

*Correspondence:

Ovidiu Vermesan

Ovidiu.Vermesan@sintef.no

Specialty section:

This article was submitted to

Transportation Systems Modeling,

a section of the journal

Frontiers in Future Transportation

Received: 30 March 2021

Accepted: 29 June 2021

Published: 26 August 2021

Citation:

Vermesan O, John R, Pype P,

Daalderop G, Kriegel K, Mitic G,

Lorentz V, Bahr R, Sand HE,

Bockrath S and Waldhör S (2021)

Automotive Intelligence Embedded in Electric Connected Autonomous and

Shared Vehicles Technology for

Sustainable Green Mobility.

Front. Future Transp. 2:688482.

doi: 10.3389/ffutr.2021.688482
The automotive sector digitalization accelerates the technology convergence of perception, computing processing, connectivity, propulsion, and data fusion for electric connected autonomous and shared (ECAS) vehicles. This brings cutting-edge computing paradigms with embedded cognitive capabilities into vehicle domains and data infrastructure to provide holistic intrinsic and extrinsic intelligence for new mobility applications. Digital technologies are a significant enabler in achieving the sustainability goals of the green transformation of the mobility and transportation sectors. Innovation occurs predominantly in ECAS vehicles' architecture, operations, intelligent functions, and automotive digital infrastructure. The traditional ownership model is moving toward multimodal and shared mobility services. The ECAS vehicle's technology allows for the development of virtual automotive functions that run on shared hardware platforms with data unlocking value, and for introducing new, shared computing-based automotive features. Facilitating vehicle automation, vehicle electrification, vehicle-to-everything (V2X) communication is accomplished by the convergence of artificial intelligence (Al), cellular/wireless connectivity, edge computing, the Internet of things (IOT), the Internet of intelligent things (IolT), digital twins (DTs), virtual/augmented reality (VR/AR) and distributed ledger technologies (DLTs). Vehicles become more intelligent, connected, functioning as edge micro servers on wheels, powered by sensors/actuators, hardware $(\mathrm{HW})$, software (SW) and smart virtual functions that are integrated into the digital infrastructure. Electrification, automation, connectivity, digitalization, decarbonization, decentralization, and standardization are the main drivers that unlock intelligent vehicles' potential for sustainable green mobility applications. ECAS vehicles act as autonomous agents using swarm intelligence to communicate and exchange information, either directly or indirectly, with each other and the infrastructure, accessing independent services such as energy, high-definition maps, routes, infrastructure information, traffic lights, tolls, parking (micropayments), and finding emergent/intelligent solutions. The article gives an overview of the advances in Al technologies and applications to realize intelligent functions and optimize vehicle performance, control, and decision-making for future ECAS vehicles to support the acceleration of deployment in various mobility scenarios. 
ECAS vehicles, systems, sub-systems, and components are subjected to stringent regulatory frameworks, which set rigorous requirements for autonomous vehicles. An in-depth assessment of existing standards, regulations, and laws, including a thorough gap analysis, is required. Global guidelines must be provided on how to fulfill the requirements. ECAS vehicle technology trustworthiness, including Al-based HW/SW and algorithms, is necessary for developing ECAS systems across the entire automotive ecosystem. The safety and transparency of Al-based technology and the explainability of the purpose, use, benefits, and limitations of Al systems are critical for fulfilling trustworthiness requirements. The article presents ECAS vehicles' evolution toward domain controller, zonal vehicle, and federated vehicle/edge/cloud-centric based on distributed intelligence in the vehicle and infrastructure level architectures and the role of Al techniques and methods to implement the different autonomous driving and optimization functions for sustainable green mobility.

Keywords: artificial intelligence, electric connected autonomous and shared vehicles, internet of vehicles, edge computing, automotive architectures, swarm intelligence, vehicle-to-everything communication, green mobility

\section{INTRODUCTION}

Climate change, global warming, ecological and environmental degradation are global existential threats. Consequently, the new European Green Deal (European Commission, 2019a) roadmap entails a growth strategy to transform Europe into a modern, resource-efficient, and competitive economy. The roadmap aims to transform the economy to achieve climate neutrality by 2050 . The transformation can be done by "turning climate and environmental challenges into opportunities across all policy areas and making the transition just and inclusive for all" (European Commission, 2019a).

The European Green Deal is an essential part of the EC's strategy to implement the UN's 2030 Agenda (United Nations, 2015a) and its sustainable development goals (United Nations, 2015b). To implement this strategy, the European Union has adopted a mobility action plan based on the Vision Zero and Safe System approach (European Commission, 2019b) (zero accidents, zero pollution, and zero congestion). The Green Deal defines four critical elements for sustainable mobility and the automotive industry: climate neutrality, zero pollution Europe, sustainable transport, and the transition to a circular economy. The circular economy action plan (European Commission, 2020) has detailed measures to make sure that sustainable products are the norm in the EU. This plan puts a primary focus on "digital technologies" such as electronics, ICT, and energy storage systems (e.g., batteries, supercapacitors, fuel cells, etc.), which can result in an increase in the lifetime, availability and usage of future vehicles based on AI-enabled technologies.

Digital technologies are a significant enabler for attaining the European Green Deal's sustainability goals in many different sectors, including mobility and transportation. Digital technologies such as edge computing, IoT, AI, cellular/wireless connectivity, DTs, VR/AR and DLTs can accelerate and maximize the impact of policies that deal with climate change and protect the environment by developing new sustainable electronic component and systems technologies for future vehicles. Expanding automotive intelligence at the vehicle and mobility system level allows the Internet of Vehicles (IoV) and Internet of Energy (IoE) (Vermesan et al., 2011) to become the key enabling technologies to realize future autonomous driving scenarios that embed cognition and autonomous functions.

Performing the driver tasks like a human driver or better requires using $\mathrm{AI}$ technologies and efficient algorithms to solve problems that include many heterogeneous participants in the traffic, operating in highly dynamic environments, and encountering many uncertainties. The OEMs deploying AI must deploy the technology in a trustworthy manner and actively supporting an ethical approach to AI, one based on a recognized set of values and principles to be enshrined into industry-led initiatives (ACEA, 2020; AECC, 2021; AVCC, 2019).

The ECAS vehicles have to match or even outperform the basic five human senses of sight, hearing, taste, smell, and touch to provide driver's capabilities. ECAS vehicle must implement several of these senses to allow the vehicle to interact with other vehicles, the traffic participants, and operate safely in the physical environment. Human intelligence's fundamental components include creativity, intuition, knowledge, experience, reasoning, memory, learning ability, creativity, intuition, reflexes, coordination, and emotions. Several of these intelligence components are relevant for autonomous driving functions. Knowledge is acquired through the analysis of information, and experience is gained through the accumulation of knowledge over time. The ECAS vehicles must acquire knowledge as the knowledge will increase their intelligence, supporting the reasoning and learning ability, so the vehicles individually and collectively, through logical reasoning, convert information into knowledge, make decisions and solve problems. Advanced concepts based on $\mathrm{AI}$ are needed to create ECAS systems to detect, predict, and react to other road user's behavior, including other vehicles, pedestrians, and animals. An overview of the autonomous vehicle intelligence and senses compared with the driver capabilities is presented in Figure 1. As explained later in this chapter, the ECAS vehicles must 


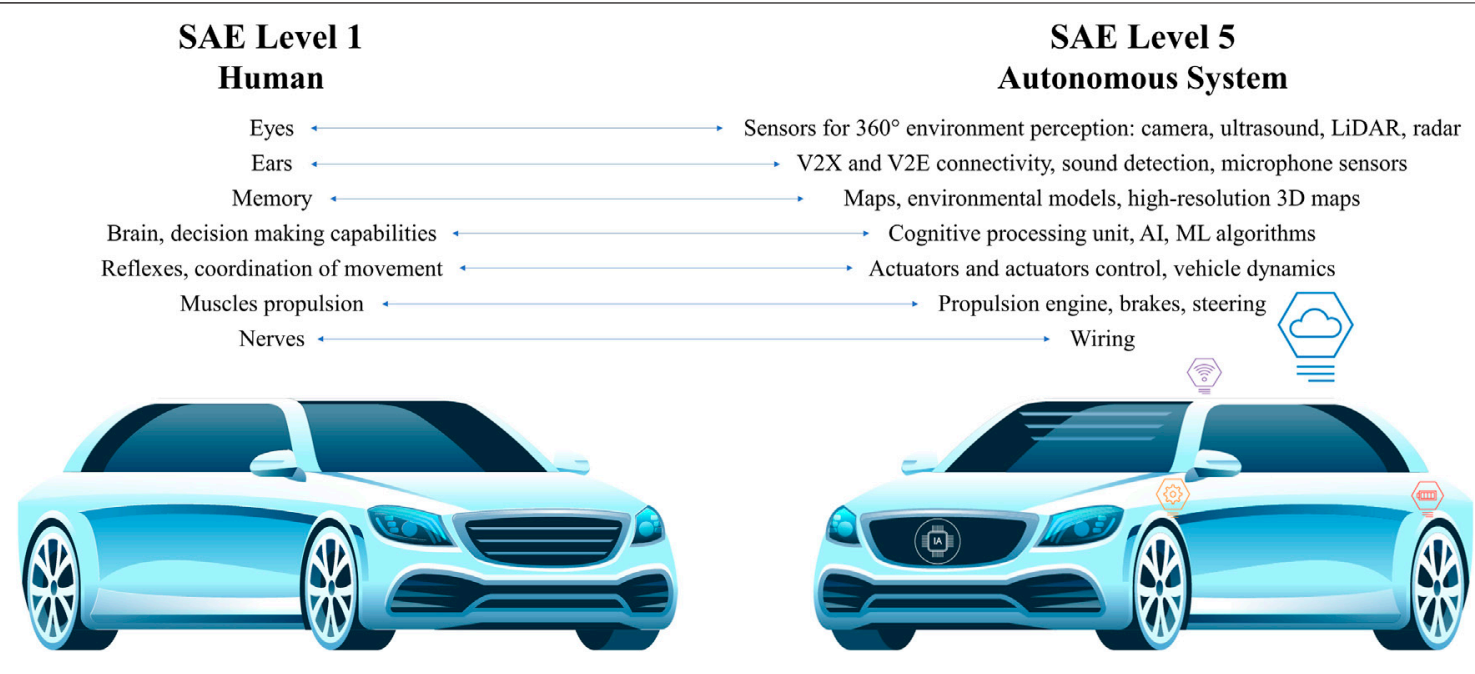

FIGURE 1 | Autonomous vehicle intelligence and senses compared with the driver capabilities.

be able to LOCATE, SENSE, COMPREHEND, THINK, CONNECT, ACT, COLLABORATE and LEARN to match and outperform the human driver capabilities. AI augment and replace human activity in ECAS vehicles. The first phase is implemented by OEMs using an augmented approach whereby AI provides assistance and controlled automation to prepare future autonomous vehicles.

In this context, a discussion of the AI definitions, characteristics, and capabilities is necessary as future automotive technology innovations rely on various AI forms with individual/collective/ collaborative conditions that underly the intrinsic vehicle capabilities. AI is adopted in a broader extrinsic mobility level related to virtual intelligent green mobility, transportation infrastructure, and systems-based mobility virtualization approaches leveraging in-vehicle connectivity and sensors combined with edge-based and cloud aggregation and processing.

In this article, AI is defined as the ECAS vehicle and its systems' abilities to perform logical analysis, sense, acquire knowledge, reason, make decisions, learn and adapt to an environment that varies over time and/or in a given context. These capabilities include the collective attributes of ECAS vehicle systems, intelligent IoT devices capable of performing functions such as learning, decision making, or other intelligent behaviors. Several definitions of AI and machine intelligence are presented in Vermesan et al. (2020b).

Three types of AI are defined in Pohl (2015), Artificial Narrow Intelligence (ANI), Artificial General Intelligence (AGI), and Artificial Superintelligence (ASI). The ANI referred to as weak AI (Searle, 1998) focuses on specific, narrowly defined tasks solved by algorithms and includes voice, language, patterns recognition, and machine vision used in autonomous vehicles relying on DL and ML technologies. AGI is referred to as strong AI (Searle, 1998) has an intelligent agent's capabilities to understand and learn any rational, intellectual task that a person can. The AGI can reach the human's intelligence level in several domains such as reasoning, comprehending complex ideas/situations, planning, solving problems, thinking abstractly, learning fast from experience. The AGI is expected to be achieved in the future for providing full autonomous vehicle capabilities. ASI is described as the level of AI reached by machines when their abilities exponentially surpass a human's intelligence level by several magnitude orders. Bostrom (2014) argues that if machine brains exceed human brains in intelligence, this new superintelligence could replace humans as the dominant lifeform on Earth. Intelligent machines could improve their capabilities faster than human-computer scientists, and the outcome could be existential trouble for humans. The argument for the emergence of artificial superintelligence is convincing (Thomas, 2016). Expanding the cognitive limitations to our empirically assessing AI risk is valid as the philosophical, epistemic claim includes significant ramifications for contemporary society our limits in gauging risk scientifically.

The AI technologies applied to ECAS vehicles and mobility need to address the intrinsic and extrinsic capabilities at the vehicle and application/mission level, considering both the individual and collective intelligence. The collective perception intelligence is sustained by developing intelligent connectivity $\mathrm{V} 2 \mathrm{X}$ and the decentralized and distributed evolutionary computing based on IoIT, edge and swarm computing federated to the cloud. In a holistic future, intelligent green mobility is an extensive interconnected system of systems, collective and continuously learning as they perform their missions. Internet of Vehicles systems will act as fleets with embedded swarm intelligence and AI algorithms.

The paper is organized as follows. The introduction is included in Introduction. The concept and evolution of ECAS vehicles are presented in Concept and Evolution of Electric Connected Autonomous and Shared Vehicles. The overview of the next generation ECAS vehicle architectures is presented in ECAS Vehicle Architectures. ECAS Vehicle Domains and Zones Concepts is dedicated to describing the ECAS vehicles domains and zones concepts, focusing on the functions of the AI components and cognitive functions at different vehicle functional domains and 
zones. The section will present the technologies developments to address battery management, energy recovery, vehicle-to-grid (V2G) mode of operation to increase the network's storage capacity and increase renewable energy utilization. Automotive Intelligence Design Issues and AI Techniques and Methods for the Implementation of the ECAS Vehicles addresses the design issues in implementing the ECAS vehicles to advance the safety and performance of the traffic system. The section presents the advances in the perception sensor fusion, advanced connectivity for cooperative mobility applications, vehicle/edge/cloud computing integration concepts, new digital platforms for efficient and federated computing, and intelligent components based on trustworthy AI techniques and methods. Intrinsic and Extrinsic Intelligence for Sustainable Green Mobility explores the concept of intrinsic and extrinsic intelligence applied to the convergence of technologies such as IoT, edge computing, swarm intelligence (Vermesan et al., 2020a) applied to future sustainable green mobility. Future Research Challenges in Automotive Intelligence discusses future research challenges and presents key open issues related to AI techniques and methods in the implementation of ECAS vehicles. The summary and conclusions are given in Summary and Conclusions.

\section{CONCEPT AND EVOLUTION OF ELECTRIC CONNECTED AUTONOMOUS AND SHARED VEHICLES}

In the mobility and transportation sector, the digital economy's transition can be realized through new applications and services and new business models that leverage digital technology advances.
Electrification, automatization, digitalization, and standardization are trends that support the digital transformation. These mobility trends translate in the drive for ECAS vehicles.

The entire traditional automotive ecosystem is transforming to address the new technologies, applications, and mobility modes. The new digital technologies impact the industry and consumers and bring new requirements for higher safety, security, privacy, the efficient electrification of the powertrain, and the introduction of data analytics and AI techniques for deploying intelligent solutions and components.

The role of AI is significant to ECAS vehicle's future to provide the technology in the different vehicle domains and the AI applications that extend beyond engineering, production, supply chain to customer experience, mobility services, and business models. The technology, dependability/ trustworthiness, regulations, and standardization are keys to achieving sustainable green mobility using next-generation ECAS vehicles.

The main elements behind the development of ECAS vehicles are presented below and illustrated in Figure 2.

\section{Electric}

The transition from internal combustion engine (ICE) vehicles to $100 \% \mathrm{EVs}$ is one way of addressing global climate change, pollution, limited resources, and the need to improve the efficiency of the propulsion systems. EVs are not entirely emission-free, but they have much lower $\mathrm{CO}_{2}$ emission and have clear potential to lower $\mathrm{CO}_{2}$ emissions close to zero when using local renewable energy sources. The massproducing vehicle manufacturers are shifting the production to conceive new EV designs and meet dynamic consumer demands.

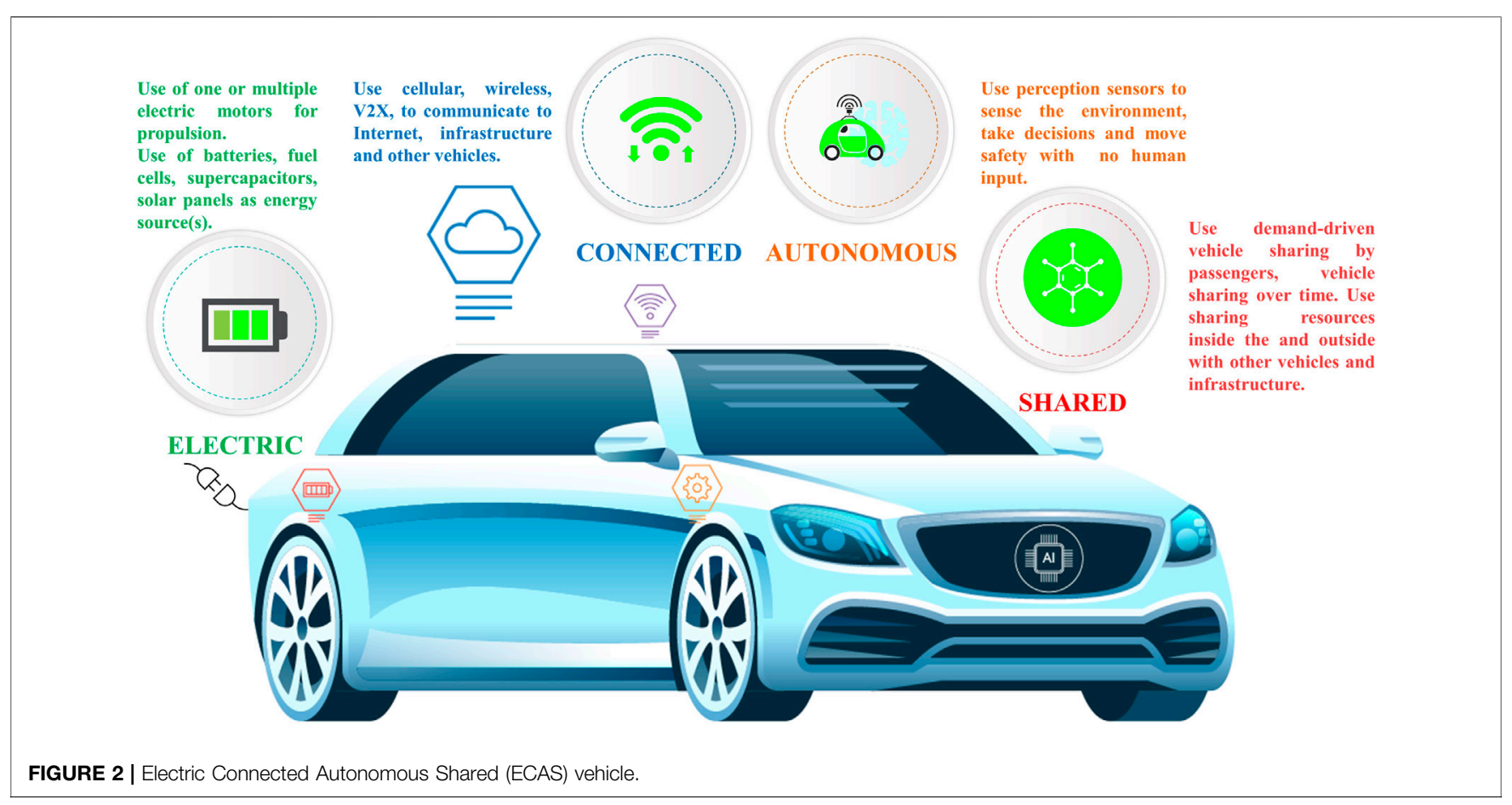


Building entirely new fleets of EVs on a mass scale depends on the rollout of entirely new infrastructures. Intelligent charging network infrastructures need to be implemented to eliminate "range anxiety" for vehicle users, reducing the recharging time and optimizing the charging and energy-efficient use of renewables. The use of AI-based electric/electronics hardware/ software architectures and platforms allows for implementing new energy storage solutions and services. Electrification of vehicles opens an entire infrastructure sector that can be built around charging stations throughout communities, wireless charging roads, and to a greater extent, vehicle sharing technologies. Electrification accelerates the development of autonomy in public transit and mobility business models.

\section{Connected}

The connected vehicles interact with the surrounding environment and infrastructure using two-way intelligent cellular/wireless connectivity capabilities to exchange real-time data to other vehicles, mobility solutions stakeholders, the edge/cloud computing infrastructure, and provide over-the-air function updates and upgrades. Advanced vehicle connectivity represents a critical element supporting the mobility sector's digitalization and implementing shared economy services and applications. Whereas communication and radar technologies are already found separately, the new technological developments show a convergence of radar-communication technologies in mobility applications. Examples are integrated radar sensors/ communication modules on traffic lights, radar and image sensor fusion between vehicles via direct communication, beamforming for direct communication to vehicles in the immediate vicinity, warnings to vulnerable road users (VRU) based on (multiple) radar sensors. The new connectivity technologies allow the vehicles to share internet access, and hence data, with other vehicles, infrastructure, people, and devices both inside and outside the vehicle. For safety-critical applications, it is anticipated that vehicles are connected using dedicated communications networks for V2X applications. Such networks must be ad-hoc networks (e.g., capable of working without the need for an infrastructure). The increased connectivity raises the threat of malicious hackers and compromised cybersecurity (Kim et al., 2020), increasing the need for more advanced in-vehicle and edge/cloud-based safe, secure, reliable, and un-hackable components, embedded software, and systems. V2X communication technologies are used to mitigate traffic collisions and improve traffic congestion by exchanging basic safety information (location, speed, and direction between vehicles within range of interaction). The V2X technologies extend active safety features and are a fundamental component of autonomous driving. Intelligence connectivity allows the exchange of sensor and awareness data among vehicles, cooperative localization, and map updating and facilitates coordinated maneuvers between autonomous vehicles.

\section{Autonomous}

Autonomous vehicles can sense the environment and drive safely with minimum or no human input. There are different autonomy levels. SAE International produced a chart designed to clarify the
J3016 "Levels of Driving Automation" standard, which defines six driving automation levels, from SAE Level 0 (SAE L0 no driving automation) to SAE Level 5 (SAE L5 full driving automation) (SAE International, 2019). SAE defines motor vehicle driving automation systems that perform part or all the dynamic driving task (DDT) on a sustained basis. The standard specifies that "a given vehicle may be equipped with a driving automation system capable of delivering multiple driving automation features that perform at different levels". "The level of driving automation exhibited in any given instance is determined by the feature(s) that are engaged" (SAE International, 2019). Terms such as conditional automation, high automation, full automation, automated, autonomous, driverless, self-driving, or fully autonomous are used to describe the SAE Level 3 (SAE L3) to SAE L5 vehicles. In the current literature, there are inconsistencies in the terminology used for the self-driving/driverless automated/ autonomous vehicles, and various organizations and stakeholders have proposed to define an accurate and consistent vocabulary (SAE International, 2019; European Union, 2019). In this article, the authors utilize the term autonomous instead of automated. The term autonomous reflects that the vehicles act independently, are self-governing their operations, are self-aware, and capable of making various choices in specific scenarios and missions, including AI-based cognitive behavior, planning, and decision making. The autonomous driving benefits are reflected in increased safety (reduce the risk of crashes, accidents, and injuries), greater productivity, less congestion (resulting in less pollution), and improved fuel economy. The autonomous functions deployment expects increased lane capacity on roads, a significant drop in harmful emissions, reduced travel time, and considerable consumer savings. The autonomous vehicles operating as part of the IoV paradigm can create new mobility business models, new mobility solutions, and services (Autonomous vehicles, 2019). Because in SAE Level 5, the "driver" does not have any responsibility for the operation, there are no special driving skills needed. Hence the involved hardware must ensure fail-operational functions at any time (Vermesan et al., 2021a). This means the reliability of the systems is crucial.

\section{Shared}

The mobility solutions are swiftly deploying new forms of vehiclesharing, ride-sharing/lift-sharing, and ride-hailing services that are changing the vehicle's ownership model, transforming the demand throughout the day, improving access to mobility by lowering the resources to participate, and increasing utilization rates of the individual vehicles and vehicle fleets assets. Shared mobility relates to demand-driven vehicle sharing by passengers ride-sharing/liftsharing (sharing a vehicle as a group simultaneously) or vehicle sharing (over time) as a rental or short-term for a trip. Shared mobility services are practicing energetic and positive steps to integrate fully into public transportation systems and communities. Seamless multimodal experiences allow the users to focus on where to go and less on how to combine a set of disparate mobility solutions to arrive there. Shared and multimodal mobility services need to be combined with supported privacy standards to avoid potential impacts caused by the General Data protection regulation 
TABLE 1 | Overview of standards relevant to ECAS vehicles.

Standardization organizations

Standards

Description and comments

AEC - Automotive Electronics Council

AEC Q100 (2014)

Failure mechanism-based stress test qualification for integrated circuits, (2014): Covers failure mechanism-based stress tests, minimum stress test-driven qualification requirements, and test conditions for qualifying integrated circuits (ICs). The idea is to determine devices that can pass the defined stress tests and provide devices that can offer a certain quality and reliability level in the application. Defines four temperature ranges based on the operating range of ICs. Other related documents are AEC Q101 (discrete semiconductors such as diodes, transistors, etc.), AEC Q102 (optoelectronic semiconductors), AEC Q103 (MEMS pressure sensor devices), AEC Q104 (multichip modules), and AEC Q200 (passive components)

\section{ANSI/UL - American National Standards Institute/Underwriters Laboratories}

ANSI UL $4600(2020)$

Standards for Safety - Evaluation of autonomous products (2020): This standard's scope is a generalized autonomous system standard framework using autonomous vehicles as an example. The approach requires a claim-based safety case that encompasses the entirety of the material necessary for safety assurance. The safety case includes a structured set of claims, arguments, and evidence supporting the proposition that items like autonomous vehicles and supporting infrastructure are acceptably safe for deployment.

\section{CEN - European Committee for Standardization}

Identification of vehicles and infrastructures compatibility - Graphical expression for consumer information on the EV power supply (2019): Address harmonized identifiers for electric vehicle's power supply (EV). The requirements are to complement user's informational needs regarding the compatibility between the EV charging stations, the cable assemblies, and the vehicles that are placed on the market. The identifier is intended to be visualized at EV charging stations, on vehicles, on cable assemblies, in EV dealerships, and consumer manuals.

\section{CEN/ISO - European Committee for Standardization/International Organization for Standards}

EN ISO 17409 (2020)

EN ISO 17427-1 (2018)

EN ISO 18541-6 (2018)

EN ISO $19363(2021)$

EN ISO 24534 Parts (2010-2019)

EN ISO 24978 (2009)
Electrically propelled road vehicles - Conductive power transfer - Safety requirements (2020): Specifies electric safety requirements for electric vehicles' conductive connection to external electric circuits, including external electric power supplies and external electric loads. Provides requirements for charging modes 2, 3, 4 (IEC 61851-1) and reverse power transfer, plus requirements regarding the connection to an isolated DC EV charging station for mode 4 (IEC 61851-23). Intelligent transport systems - Cooperative ITS - Part 1: Roles and responsibilities in the context of co-operative ITS architecture(s), (Part 1, 2018): Detailed description of the roles and responsibilities required to deploy and operate Cooperative-ITS (C-ITS). The organization described in this document is designed to be appropriate for any fully operational system that uses the C-ITS concepts and techniques to achieve its service provision.

Road vehicles - Standardized access to automotive repair and maintenance information (RMI) - Part 6: L-Category vehicle specific RMI use cases and requirements, (2018): Address elements like definitions, use cases, technical requirements, functional user interfaces requirements, and conformance test cases applicable for the standardized access to repair and maintenance information for vehicles, (development based on ISO 1854 Parts 1-4). References the usage of a Digital Annex of standardized search terms for RMI.

Electrically propelled road vehicles - Magnetic field wireless power transfer - Safety and interoperability requirements (2021): Define the onboard vehicle equipment's requirements and operation that enables magnetic field wireless power transfer (MF-WPT) for traction battery charging of electric vehicles. Address aspect as safety requirements, transferred power and efficiency, ground clearance of the EV device, functionality with associated off-board systems under various conditions, independent of the manufacturer, and test procedures. Intended to operate with supply devices that fulfill the MF-WPTrelated requirements in IEC 61980.

Automatic vehicle and equipment identification - Electronic Registration Identification (ERI) for vehicles, (Part 1-4, 2010-2019): Provides requirements for electronic registration identification (ERI) that are based on an identifier assigned to a vehicle (e.g., for recognition by national authorities), and adheres to privacy and data protection regulations. Part 1 (architecture) provides an overview of the ERI system concept regarding the onboard vehicle components and the external off-vehicle components required for an operational system. The detailed requirements are defined in Part 2 (operational requirements), Part 3 (intelligent transport systems), and Part 4 (secure communications using asymmetrical techniques). Intelligent transport systems - ITS Safety and emergency messages using any available wireless media - Data registry procedures, (2009): Address intelligent transport systems and provides a standardized set of protocols, parameters, and a method of management of an updateable "Data Registry" to provide application layers for "ITS Safety messages" using any available wireless connectivity.

\section{IATF - International Automotive Task Force}

IATF 16949 (2016)

Technical specification - Quality management systems for automotive production and relevant service parts organizations, (2016): Defines the quality management system (QMS) requirements for automotive production, service, and accessory parts. An autonomous QMS standard is aligned with the structure and requirements of ISO 9001 and implemented as a supplement.

(Continued on following page) 
TABLE 1 | (Continued) Overview of standards relevant to ECAS vehicles.

\section{Standardization organizations}

Standards

IEC - International Electrotechnical Commission

Description and comments

IEC 61508 (2010)

IEC 61851-1 (2017)

IEC 61851-23 (2014)

IEC 61851-24 (2014)

IEC 62752 (2016)

ISO/IEC TR 24029-1 (2021)

ISO/IEC AWI 24029-2 (2021)

ISO/IEC 27001 (2013)

Functional safety of electrical/electronic/programmable electronic safety-related systems (Part 0-7, 2010): Provides functional safety standards for the lifecycle of electrical, electronic, or programmable electronic (E/E/PE) systems and products. It addresses those parts that perform automated safety functions (e.g., sensors, control logic, actuators, and microprocessors) and defines four Safety Integration Levels (SIL).

Electric vehicle conductive charging system - Part 1: General requirements. The standard applies to EV supply equipment for charging electric road vehicles, with a rated supply voltage up to 1,000 V AC or up to 1,500 V DC. and a rated output voltage up to $1,000 \vee \mathrm{AC}$. Or up to $1,500 \mathrm{~V}$ DC. Electric road vehicles (EV) cover all road vehicles, including plug-in hybrid road vehicles (PHEV), that derive all or part of their energy from on-board rechargeable energy storage systems (RESS). Th standard also applies to EV supply equipment supplied from on-site storage systems (e.g., buffer batteries).

Electric vehicle conductive charging system - Part 23: DC electric vehicle charging station. The standard gives the requirements for d.c. electric vehicle (EV) charging stations, herein also referred to as "DC charger", for conductive connection to the vehicle, with an a.c. or d.c. input voltage up to 1,000 V a.c. and up to 1,500 V d.c. according to IEC 60038 It provides the general requirements for the control communication between a d.c. EV charging station and an EV Electric vehicle conductive charging system - Part 24: Digital communication between a d.c. EV charging station and an electric vehicle for control of d.c. charging. The standard applies to digital communication between a d.c. EV charging station and an electric road vehicle (EV) for control of d.c. charging, with an a.c. or d.c. input voltage up to 1,000 V a.c. and up to $1,500 \mathrm{~V}$ d.c. for the conductive charging procedure.

In-cable control and protection device for mode 2 charging of electric road vehicles (IC-CPD). The standard is applied to incable control and protection devices (IC-CPDs) for mode 2 charging of electric road vehicles, hereafter referred to as IC-CPD including control and safety functions. The standard is applied to portable devices performing simultaneously the functions of detection of the residual current, of comparison of the value of this current with the residual operating value and of opening of the protected circuit when the residual current exceeds this value.

Artificial Intelligence (Al) - Assessment of the robustness of neural networks - Part 1: Overview.

Artificial Intelligence (Al) - Assessment of the robustness of neural networks - Part 2: Methodology for the use of formal methods (under development).

Information Security Management. Information technology - Security techniques - Information security management systems - Requirements. The standard defines the requirements for establishing, implementing, maintaining, and continually improving an information security management system within the context of the organization. It includes requirements for the assessment and treatment of information security risks tailored to the needs of the organization.

IEEE SA- Institute of Electrical and Electronics Engineers Standard Association

IEEE 802.11p (2010)

IEEE P802.11bd (2018)

IEEE 1609 Parts (2006-2020)

IEEE P1228 (2019)

IEEE P2020 (2016)

IEEE P2846 (2020)
IEEE Standard for Information Technology - Local and metropolitan area networks - Specific requirements -- Part 11: Wireless LAN Medium access control (MAC) and Physical layer (PHY) Specifications amendment 6: Wireless access in vehicular environments, (2010): This amendment specifies the extensions to IEEE 802.11 standard for wireless local area networks (WLANs) providing wireless communications while in a vehicular environment.

Standard for Information Technology - Telecommunications and information exchange between systems local and metropolitan area networks - Specific requirements - Part 11: Wireless LAN Medium access control (MAC) and Physical layer (PHY) Specifications amendment: Enhancements for next generation V2X.

Standards for Wireless Access in Vehicular Environments (WAVE), (Family of standards): A family of standards within intelligent transport systems (ITS), defining the architecture, services, and standard interfaces for secure V2V and V2I wireless communications. Applications may utilize these standards in conjunction with $5.9 \mathrm{GHz}$ radio equipment to provide, for example, services to drivers, operators, and maintenance personnel. This family of standards relies on the IEEE P802.11p standard.

Standard for Software Safety that is relevant to software safety during the development, procurement, maintenance, and retirement of safety-critical software (e.g., software products whose failure could cause loss of life, serious harm, or have widespread negative social impact). The standard requires that software safety be considered within the context of the system safety program throughout the software lifecycle and addresses only the safety aspects of the software. The document discusses aspects of software safety related to interoperation with other systems or constituents of a system of systems.

Standard for Automotive System Image Quality (Project, 2016): Address fundamental attributes that contribute to image/ quality for automotive Advanced Driver Assistance Systems (ADAS) applications and identifying existing metrics and other useful information relating to these attributes. Define a standardized suite of objective and subjective test methods for measuring automotive camera image quality attributes. Specify tools and test methods to facilitate standard-based communication and comparison among Original Equipment Manufacturer (OEM) and Tier 1 system integrators and component vendors.

Assumptions for Models in Safety-Related Automated Vehicle Behavior (Project, 2020): Describes the minimum set of reasonable assumptions used in foreseeable scenarios to be considered for road vehicles in the development of safetyrelated models that are part of automated driving systems. The standard includes rules consideration of the road and their regional and temporal dependencies.

(Continued on following page) 
TABLE 1 | (Continued) Overview of standards relevant to ECAS vehicles.

\section{Standardization organizations}

Standards

IEEE P2851 (2019)

\section{Description and comments}

Standard for Exchange/Interoperability Format for Functional Safety Analysis and Functional Safety Verification of IP, SoC and Mixed-Signal ICs, (Project, 2019): Defines a data format with which results of functional safety analyses such as Failure mode and effects analysis (FMEA), Failure modes, effects and diagnostic analysis (FMEDA), Failure mode, effects and criticality analysis (FMECA), and Fault tree analysis (FTA). The standard's goal is to provide a common ground for Electronic design automation (EDA), SoC, and IP vendors to develop tools for functional safety-critical applications.

\section{IPC - IPC International Inc}

IPC 2551 (2020)

International Standard for Digital Twins, (2020): Part of the IPC Factory of the future standards establishing the IPC digital twin, which comprises the digital twin product, manufacturing, and lifecycle frameworks. The standard stipulates/defines digital twin properties, types, complexities, and readiness levels and includes historical information.

ISO - International Organization for Standards

ISO 19206-2 (2018)

Road vehicles - Test devices for target vehicles, vulnerable road users and other objects, for assessment of active safety functions - Part 2: Requirements for pedestrian targets. The standard defines the properties of pedestrian targets that represent an adult or a child in terms of size, shape, reflection properties, etc. for testing purposes. The standard addresses the detection requirements for a pedestrian target in terms of sensing technologies commonly in use at the time of publication of this document, and where possible, anticipated future sensing technologies. The document presents methodologies to verify the target response properties to these sensors, as well as some performance requirements for the target carrier. The standard does not address the test procedures in terms of speeds, positions, or timing of events and performance criteria for the active safety system being evaluated.

ISO 19206-3 (2021)

Road vehicles - Test devices for target vehicles, vulnerable road users and other objects, for assessment of active safety functions - Part 3: Requirements for passenger vehicle 3D targets (under development).

ISO 19206-4 (2020)

Road vehicles - Test devices for target vehicles, vulnerable road users and other objects, for assessment of active safety functions - Part 4: Requirements for bicyclist targets. The standard establishes the detection requirements for a bicyclist target (BT) in terms of sensing technologies commonly in use at the time of publication of this document, and where possible, anticipated future sensing technologies. It also establishes methodologies to verify the target response properties to these sensors, as well as some performance requirements for the target carrier. The BT is also representative for electrically assisted pedal bicycles (pedal electric cycle, pedelec). The standard does not address the test procedures in terms of speeds, positions, or timing of events and performance criteria for the active safety system being evaluated.

ISO $19237(2017)$ Intelligent transport systems - Pedestrian detection and collision mitigation systems (PDCMS) - Performance requirements and test procedures. The standard specifies the concept of operation, minimum functionality, system requirements, system interfaces, and test procedures for PDCMS. It defines the behaviors that are required for PDCMS, and the system test criteria necessary to verify that a given implementation meets the requirements of this document. Implementation choices are left to system designers wherever possible.

ISO $22078(2020)$

ISO $26262(2018)$ Intelligent transport systems - Bicyclist detection and collision mitigation systems (BDCMS) -Performance requirements and test procedures.

Road vehicles - Functional safety (Part 1-12, 2018): Address safety-related systems that include one or more electrical/ electronic (E/E) systems installed in series production passenger vehicles. The Automotive safety integrity level (ASIL) is a key component, and each safety requirement is assigned an ASIL of $A, B, C$, or D.

ISO PAS 21448 (2019) Road vehicles - Safety of the intended functionality (2019): SOTIF provides guidance on the applicable design, verification, and validation measures needed to achieve the intended functionality's safety. Examines whether a needed safety functionality is successfully delivered in the absence of a failure, such as performance limitations (e.g., systems, subsystems, sensors, unexpected road environment changes).

ISO/DIS $22737(2020)$ Intelligent transport systems - Low-speed automated driving (LSAD) systems for predefined routes - Performance requirements, system requirements and performance test procedures. The standard specifies requirements for operational design domain, system requirements, minimum performance requirements and performance test procedures for safe operation of Low-speed automated driving (LSAD) systems which will operate on predefined routes. Low-speed automated driving (LSAD) systems are designed to operate at Level 4 automation, within specific Operational design domains (ODD). Road vehicles - Safety and cybersecurity for automated driving systems - Design, verification, and validation, (2020): Address steps for developing and validating automated driving systems based on basic safety principles. Considers safety-/ cybersecurity-by-design and verification/validation methods for automated driving systems focused on vehicles with SAE level 3 and level 4 (SAE J3016). Outline cybersecurity considerations are intersecting with objectives for the safety of automated driving systems.

ISO 8855 (2011)
Road vehicles - Vehicle dynamics and road-holding ability-Vocabulary developed by Technical Committee ISO/TC 22, Road vehicles, Subcommittee SC 9, Vehicle dynamics and road-holding ability. The standard defines the terms applied for road vehicle dynamics. The terms are used to passenger vehicles, buses, and commercial vehicles with one or more steered axles, and to multi-unit vehicle combinations.

Continued on following page) 
TABLE 1 | (Continued) Overview of standards relevant to ECAS vehicles.

\section{Standardization organizations}

Standards

ISO 8608 (2016)

\section{Description and comments}

Mechanical vibration - Road surface profiles - Reporting of measured data. The committee responsible for the standard is ISO/TC 108, Mechanical vibration, shock and condition monitoring, Subcommittee SC 2, Measurement and evaluation of mechanical vibration and shock as applied to machines, vehicles, and structures. The standard aims for a uniform method of reporting measured vertical road profile data for either one-track or multiple-track measurements. It is used to the reporting of measured vertical profile data taken on roads, streets and highways, and on off-road terrain.

ISO/SAE - International Organization for Standards/Society of Automotive Engineers International

ISO SAE FDIS 21434 (2020)

ISO SAE $22736(2020)$
Road vehicles - Cybersecurity engineering (Under development): This is a final draft international standard (FDIS). It addresses a framework that specifies cybersecurity risk management requirements regarding engineering for concept, development, production, operation, maintenance, and decommissioning for road vehicle electrical/electronic (E/E) systems Intelligent transport systems. Taxonomy and definitions for terms related to driving automation systems for on-road motor vehicles (under development).

\section{SAE - Society of Automotive Engineers International}

SAE J1772 (2017)

SAE J2735 (2007)

SAE J2954 (2020)

SAE J3016 (2018)

SAE J3061 (2016)
SEA Electric Vehicle and Plug-in Hybrid Electric Vehicle Conductive Charge Coupler, (2017): Address the general physical, electrical, functional, and performance requirements to facilitate conductive charging of EV/PHEV vehicles. Defines a common EV/PHEV and supply equipment vehicle conductive charging method, including operational requirements and the functional and dimensional requirements for the vehicle inlet and mating connector.

V2X Communication Message Set Dictionary, (2007): Specifies a message set, and its data frames and elements, for use by vehicle-to-everything (V2X) communications system applications. The data dictionary was initially designed for use over DSRC, but this document is intended to be independent of the underlying communications protocols used to exchange data between participants in V2X applications.

Wireless power transfer for Light-Duty Plug-in/Electric Vehicles and Alignment methodology, (2020): Specifications define acceptable interoperability criteria, electromagnetic compatibility, EMF, minimum performance, safety, and testing for wireless power transfer. The specification defines various charging levels based on the levels defined for conductive AC charge levels 1, 2, and 3 in SAE J1772.

Taxonomy and Definitions for Terms Related to Driving Automation Systems for On-Road Motor Vehicles, (2018): Describes vehicle driving automation systems that perform part or all the dynamic driving tasks on a sustained basis. It provides a taxonomy with detailed definitions for six driving automation levels, ranging from Level 0 (no driving automation) to Level 5 (full driving automation).

Cybersecurity Guidebook for Cyber-Physical Vehicle Systems, (2016): A recommended practice that provides guidance on vehicle cybersecurity and existing practices in the industry, government, and conference papers. This recommended practice establishes a set of high-level guiding principles for cybersecurity-related to cyber-physical vehicle systems, including lifecycle process framework, common existing tools and methods, basic guiding principles, and foundation for further standards development activities.
(GDPR) since the information about the origin, destination, financial information is shared in these services. The vehicles require high security and privacy protection at the component, embedded software, and system-level to provide shared mobility solutions. The term "shared" has an additional meaning for the future vehicles, which is connected to sharing resources inside the vehicles and outside the vehicle with other vehicles, other participants in the traffic, with the infrastructure, sharing the data with the mobility stakeholders, sharing services, the computing, sensing, storage, and learning capabilities.

\section{Standardization Activities}

ECAS vehicles advance the long-term mobility outlook that improves safety and transport operations while lowering the environmental impact through a higher standardized and active automated ecosystem. Standardization plays a crucial role in delivering the next-generation ECAS vehicles, mobility services, and their integration with the broader green sustainable mobility systems.

Standards are developed by standard organizations (SDOs) such as CEN/CENELEC, ETSI, IEEE, ISO, ITU-T, and SAE International. Other stakeholders develop standards such as AUTomotive Open System ARchitecture (AUTOSAR, 2003) or Open AutoDrive Forum (OADF, 2021), including standards from companies such as Apple CarPlay (ACP, 2014) standard that enables a vehicle radio or head unit to be a display and a controller for an iOS device or Android Auto (AA, 2021) which is part of the Open Automotive Alliance (OAA, 2014).

In a multi-layered ecosystem working toward autonomous vehicles, the standard development organizations (SDOs) develop guidelines for aligning the activities and standardization processes to identify the overlaps, duplication, and gaps.

The standards developed by the different SDOs, and stakeholders fall under different categories such as connectivity/connected vehicles technology/applications, awareness, localization, safety, intelligent transport systems, artificial intelligence, etc.

An overview of the SDOs technical committee's standardization activities and the standards developed relevant to ECAS vehicles are provided in Table 1. Different working groups and technical committees (TC) created within the SDOs develop the standards to address the various topics. For example, the ISO/TC 204 working group addresses the standardization of 


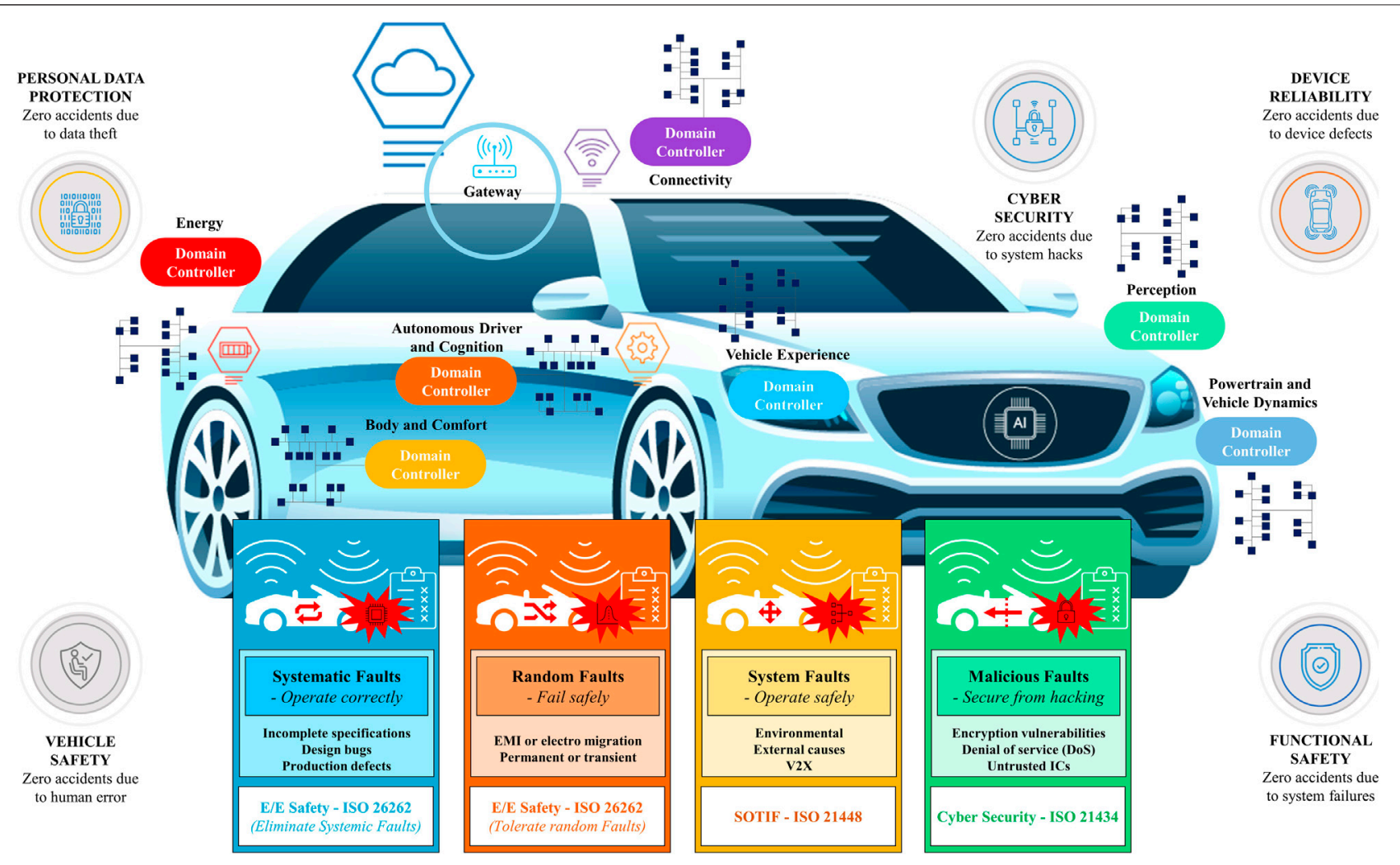

FIGURE 3 | Different failure types and faults in various standards.

information, communication, and control systems in the area of urban and rural road transportation, including intermodal and multimodal aspects thereof, traveler information, traffic management, commercial transport, public transport, emergency services, and commercial services in the intelligent transport systems (ITS) area. ISO/TC 22 produces standards for all components and systems for Road Vehicles. The ISO/TC 22 includes the automated driving ad-hoc group (ADAG). The ITU$\mathrm{T}$ focus group on $\mathrm{AI}$ for autonomous and assisted driving (FGAI4AD) working on pre-standardization studies for driving tests for AI systems and supports standardization activities for services and applications enabled by AI systems in autonomous and assisted driving. The FG-AI4AD center on the behavioral evaluation of AI responsible for the dynamic driving task. The focus group on Vehicular Multimedia (FGVM) works to identify the requirement for new vehicular multimedia standards based on space and terrestrial networks integration. The work addresses the analysis and identification of gaps in the vehicular multimedia standardization landscape to draft technical reports and specifications addressing vehicular multimedia use cases, requirements, applications, interfaces, protocols, architectures, and security. The group approaches pre-standardization studies for next-generation vehicle infotainment systems, with features like digital displays on vehicle windshields.

IEEE Standards Association (IEEE-SA) is sketching an industry connection activity to assess standardization gaps for safe automated driving to analyze, identify and assess existing standards and ongoing standardization activities and connect with the relevant SDOs. ISO and IEC started a project on AI standardization by founding the subcommittee ISO/IEC JTC 1/SC 42 Artificial Intelligence that addresses the standardization related to AI.

Aligning the standards and standardization activities are very important for the future development of ECAS vehicles. For example, ISO/PAS 21448 (2019) SOTIF is highly relevant to different driving scenarios from a systems perspective. Safety and security go together as the foundations for an autonomous system. Bringing together (ISO 26262, Parts 1-12, 2018; ISO 26262, 2019; ISO/PAS 21448, 2019) SOTIF will advance the design, test, and deployment of safe autonomous systems. Addressing the different failure types and faults in various standards is illustrated in Figure 3. ISO 26262 states that two failure types must be considered during electronic system and semiconductor development. Systematic failures related to the development, manufacturing, etc., of devices and dictate the use of effective processes to minimize the risk of issues introduced during vehicle production and random failures that relate to issues that occur during the operation of a device due to various reasons including environmental effects, device malfunction, etc. Minimizing these risks involve the analysis of the device for the effect of operational faults and building systems into the device to protect against these.

The domain of ECAS vehicles is in the developing phase. New standardization activities are starting to address the gaps in the 
legacy standards to include the new connected and autonomous functions and the emerging technologies such as AI [machine learning $(\mathrm{ML})$, deep learning (DL), neural network $(\mathrm{NN})$, computer vision, etc].

\section{ECAS VEHICLE ARCHITECTURES}

AI systems use data, knowledge, and algorithms to simulate the human brain's cognitive functions and learn and solve problems independently. The ECAS vehicles are at the forefront of using AI to replicate, augment, and assist human's actions while simultaneously leveraging machine-based system's advanced reaction times.

To provide better and enhanced driving capabilities than human drivers, the ECAS vehicles must be able to LOCATE, SENSE, COMPREHEND, THINK, CONNECT, ACT, COLLABORATE and LEARN (Vermesan et al., 2021b).

For navigating through the environment, the vehicles use the LOCATE function to identify the precise location, the direction of moving, and the vehicle's relative position in relation with other vehicles, other participants in the traffic, fix objects/obstacles, and the built and road infrastructure.

The SENSE function allows the vehicles to perceive and interpret the surroundings and their environment by identifying other traffic participants, including humans, animals, moving/fix objects/obstacles, road conditions, and traffic situations.

The vehicles utilize the COMPREHEND function to analyze and perceive the traffic situations, the environment's conditions, and the relations with the other traffic participants.

The vehicle's THINK function (Berger Roland Strategy Consultants, 2014) is applied to process the information received from the different vehicle's domains (perception/ surround view, propulsion/powertrain/vehicle dynamics, energy, connectivity, body/comfort, driving assistance/autonomous, security) and plan the driving strategy.

The vehicle's CONNECT function is to connect with the environment (V2E) and to everything (V2X).

The vehicles use the powertrain, steering, and braking power to move the wheels to put the planned driving strategy and mission into practice through the ACT function by accelerating, steering, and braking.

The COLLABORATE function enables the vehicles to interact with other vehicles, participants in the traffic, environment, infrastructure (physical, digital, edge/cloud, etc.), and objects within a shared space, or close to, which generate, create, accomplish shared goals, and minimize the risk of collisions and hazardous situations. The collaborate function combines one or several features such as natural or non-natural language communication, visual interaction, safety-rated stop monitoring, instruction by demonstrations/examples, speed and separation monitoring and power/force limiting, etc.

The LEARN function implements the actions related to the vehicle's training and learning based on the experiences in traffic and different conditions/circumstances using AI-based techniques, methods, and algorithms. Training and learning are processes that are federated between edge and cloud. The vehicles can use extensive internal computing capabilities as a training/learning platform based on the data sets collected from various missions and planned trips.
The eight functions presented above impact the electric and electronic architectures of future ECAS vehicles and the transition toward cognitive computing connected intelligent platform on wheels. The perception, sensor fusion, AI, edge computing, connectivity, function's virtualization, and resources/information sharing are critical in implementing higher automation and sustainable green mobility applications.

The ECAS vehicle manufacturers are developing their electric and electronics (E/E) architectures for ECAS vehicles based on a set of requirements, following the same central concept while providing different implementations.

The current SAE L3 vehicles can detect the environment around them using an automated driving system. The vehicles can make informed choices for themselves (e.g., overtaking slower-moving vehicles), but human override is required when the vehicle cannot execute the task at hand, or the system fails. Traditional architectures for SAE L3 vehicles include application-specific electronic control units (ECUs) connected by application-specific bus systems (CAN, FlexRay, LIN, MOST) based on 1-to-1 communication.

For the new SAE L4 vehicles, new architectures need to be developed for addressing the requirements of the functions of the vehicles.

The domains-based architectures for SAE L4 vehicles include functional consolidation in domain controller units (DCUs) in addition to application-specific ECUs connected by links based on 1-to-1 and N-to-1 communication.

The transition to advanced SAE L4 vehicles will enhance the $\mathrm{E} / \mathrm{E}$ architectures by introducing zones where the domains are zonalized, using hybrid architectures combining the vehicle zones and domains followed by full zone-based architectures.

The next-generation ECAS SAE L5 vehicles need the development of software-driven architecture centralized around the vehicle processing unit (VCU) connected by selected communication links.

Globally, the transformation of the ECAS architecture can be summarized in 3 stages, as illustrated in Figure 4.

- Current flat architecture, which is unfit for future mobility.

- Logical restructuring with a service-oriented gateway controlling and steering different domains in the vehicle, enabling an autonomous vehicle.

- Physical restructuring with an AI-based central brain, serving several zones, enabling a user-defined vehicle.

The ECAS future architectures are based on the federation of HW/SW learning platforms that are physically located in different places and offer standard virtualized functions to the vehicle and its services and applications. These platforms are using the digital twin technologies extensively for modeling, simulating, and executing in real-time the vehicle functions for specific mobility services and applications in particular environments and contexts. The architecture allows virtualization of functions and integration at a different level: actuators/sensors (e.g., physical devices providing acceleration, position, image, pressure, data to the computing level and the driving modules for the actuators like valves, motors, inverters, etc.), computing/cognition/connectivity/control (e.g., sensor fusion, domain fusion, analytics, planning's and 


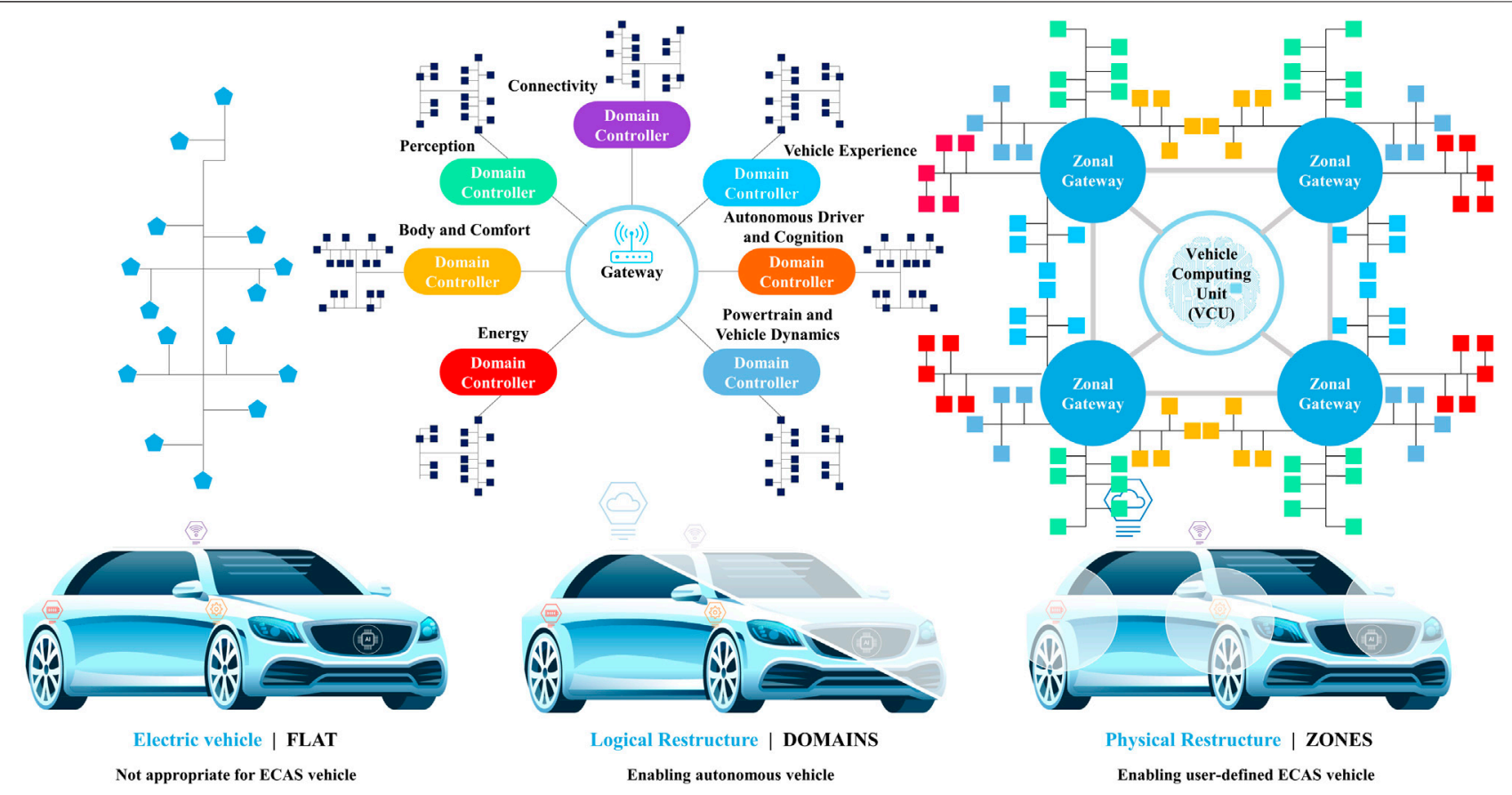

FIGURE 4 | Vehicle architecture transformation.

execution of function and services, AI learning, etc.) and edge/ cloud back-end for off-board/infrastructure computing, data management, AI training, services, etc.

The ECAS vehicle architectures demand automotive-grade embedded AI-based high-performance computing components with significant compute power while increasing energy efficiency and scalability to approach the Level 5 applications and services. The development requires accelerating the standardization of modules AI-based ECU/DCU/VCU, electronic hardware components, interfaces and software/algorithms, and APIs. The vehicle functionality enhancements through lightweight virtualization, containerization, unikernels and regular OTA updates at the platform levels utilize the benefits of separating hardware and software development cycles supporting the mobility stakeholders to speed-up software development.

The advances at the $\mathrm{E} / \mathrm{E}$ vehicle architecture level and the introduction of $\mathrm{AI}$ methods change all architecture layer's design strategies and concepts. The ECU design approaches are changing with these devices integrated into domain-specific controllers, vehicle embedded high-performance computing units, or virtualized and containerized software functions provided by the vehicle or edge. The evolution of the E/E architectures (Vermesan et al., 2015) drives the consolidation of ECUs into 6-7 domainspecific controllers for safety, perception, energy, powertrain, connectivity, body/comfort/interior, experience/infotainment. The complex autonomous driving functions demand secure, robust, energy-efficient, and real-time distributed computing resource-efficient operating systems to manage multi-tasking, coordinate operations, and eliminate excessive components. The further consolidation of ECUs into DCUs/VCUs and embedded high-performance computing platforms transform the vehicle into "intelligent connected edge computing platforms on wheels" and further into federated vehicle/edge/cloud-centric based on distributed intelligence at the vehicle and infrastructure level architectures.

\section{ECAS VEHICLE DOMAINS AND ZONES CONCEPTS}

ECAS vehicles are implemented with AI technologies to provide autonomous functions and optimize operations for implementing sustainable green mobility solutions. The ECAS vehicles are developed with cognitive capabilities comparable to that of a human brain to support the vehicles to make contextbased decisions that are not programmed in software. Performing the mobility missions allocated to them requires ECAS vehicles to locate, explore the environment by using sensors such as 360degree cameras to view the environment around, interpret this information, verify the location of the vehicle, identify the position of any traffic participant, communicate with other vehicles, infrastructure, participants in the traffic and calculate in few milliseconds, make decisions, and conduct the necessary driving maneuvers.

The perception, sensor fusion, cognition, optimization, communication, and decision-making require a large amount of computing power to provide hundreds of Tera operations per second (OPS).

To implement the electronic and computing vehicle platforms that deliver the autonomous functions' capabilities, the ECAS 
vehicles domain-based architectures are one of the logical ways to break down and cluster the hardware and software components associated with vehicle design.

The domains-based approach organizes and assembles/ connects all the functions that let vehicles locate, sense, comprehend, think, connect, act, collaborate and learn, while managing complexity and support scalability.

The main domains that are envisioned for ECAS vehicles are:

- Energy

- Powertrain and Vehicle Dynamics

- Connectivity

- Perception

- Autonomous Driver and Cognition

- Body and Comfort

- Vehicle Experience

Safety and security are addressed in all domains based on the domain requirements as part of the autonomous system dependability properties and autonomous driving trustworthiness. For example, in the connectivity domain, where the vehicle interacts with other vehicles, infrastructure and the Internet, security is critical since external communications need to be protected from tampering. In the powertrain and vehicle dynamics domain, functional safety and reliability are the main issues since the domain's components must function while exposed to extreme conditions. At the same time, security is less of a problem as the domain is isolated and operates away from external factors.

The powertrain and vehicle dynamics, body, and control are part of existing vehicle architectures. Few vehicle domains have been developed from previous architectures (e.g., infotainment, in-vehicle connectivity, propulsion, etc.). Other domains are new and relate to autonomous function's operations and AI cognitive technologie's deployment. The domain-based architectures offer an optimum level of autonomous functions, ensuring the highest safety and security degrees while paving the way to zone-based architectures and software-driven architectures centralized around the vehicle processing unit. The ECAS vehicle's architectures will sequentially evolve into federated vehicle/ edge/cloud-centric based on distributed intelligence at the vehicle and infrastructure level, need to reflect the autonomous system properties and provide characteristics such as compatibility/interoperability, composability/ modularity, scalability, and usability.

The architectures need to be optimized for various mobility missions, applications, and services. The domain-based architectures are the first optimization phase implemented for ECAS vehicles by clustering together similar functions and isolating them to design the proper safety and security (Pype et al., 2017a; Pype et al., 2017b) levels based on the common requirements within each vehicle domain.

To achieve economies of scale and lower costs, the AI-based ECAS vehicle architectures require a high level of compatibility/ interoperability to allow the substitution of the vehicle subsystems, platforms, or functional units (e.g., HW/SW, algorithms), in a relatively transparent manner, without loss of information and the introduction of errors. Interoperability between ECAS vehicle architectures to exchange information/ knowledge and use the information/knowledge that has been transferred is vital for future mobility applications, with different interoperability levels applied to the other functional domains of the ECAS vehicle architectures. The domain-based architectures open the way for compatibility/interoperability design concepts.

Composability/modularity are autonomous system properties that need to be part of the architectural concepts to distribute the driving functions into separate vehicle domains. Composability enables building the ECAS subsystems from components (SW/HW/ algorithms) providing different features, functions and assemble them (e.g., electrically, wirelessly, functionally) to implement new ECAS vehicle functions at the mobility system level. Modularity is interpreted as the extent to which the autonomous system is composed of discrete components, and changes to one part have minimal impact on other components. The modularity concept applies well to the implementation of functional safety and cybersecurity functions. It simplifies the development and implementation of autonomous driving algorithms, making it more straightforward to scale features within each subsystem.

Composability/modularity approaches allow the ECAS vehicle architecture designs to scale within each domain, from baseline performance to high-end operation, providing different features to meet various market requirements. The domain-based ECAS vehicle architectures enable scalability in each vehicle's domain and implement individual building blocks that are both compatible and reusable. The approach allows the development to be more efficient and cost-effective, based on flexible and responsive manufacturing processes.

Usability describes how systems/users can use, learn, or control the autonomous vehicle system. In this context, the concept of design-reuse can be applied by striving to use the equivalent building blocks across every automotive domain to add/increase or reduce/decrease vehicle functionality and advance the designs as new technologies are developed. The concept of design reuse by adopting common architectures, common software platforms across the vehicle architectural layers enables utilizing a standard set of tools, IP libraries, software code, and algorithms allowing the over-the-air (OTA) vehicle driving functions updates and upgrades.

An overview and a description of the ECAS vehicle domain's functions are provided in the following paragraphs.

\section{Energy Domain}

The energy domain covers the energy sources/storage units, energy harvesting/regeneration techniques, the power electronic modules, hardware/software/firmware (HW/SW/FW) control, and highlevel algorithms performing the functions of energy/power storage (battery/supercapacitors). The domain governs the overall management (cell charge/discharge/equalization functions, failure management functions, etc.) and charging/ recharging (grid connection, range extension, photovoltaic).

The developments in the vehicle energy domain have accelerated the adoption of lithium-ion batteries as the power source for ECAS vehicles, with innovations focusing 
on introducing new solid-state batteries that could provide safer, lightweight, more energy, and power-generating batteries solutions. The solid-state battery technology uses solid electrodes and a solid electrolyte instead of liquid or polymer gel electrolytes employed in lithium-ion or lithiumpolymer batteries utilized in today's electric vehicles. Challenges to the broad adoption of solid-state battery technology include material costs, stability, sensitivity, durability, energy, and power density.

Digitalization is radically transforming the energy domain, where the batteries architectures are optimizing the cables to the sensors and sensing point. Innovations like smart battery cells assembled in complex battery systems, with wireless communication between the smart cells and the central battery management system (BMS), enable ultra-high-speed interaction with the smart battery cell parameters.

The BMS is one of the central components for achieving a safe and economically viable energy storage system usage based on batteries. The BMS is playing a critical role in the reduction of costs, accurate state estimation, and increase of safety, since the BMS monitors and controls the battery to avoid safety-critical operating conditions, e.g., over-charging, over-discharging and over-current scenarios depending on external operating conditions like temperature.

Furthermore, the BMS must precisely predict the battery's state parameters like state of charge (SOC), state of energy (SOE), state of power (SOP), and state of health ( $\mathrm{SOH})$. Traditionally, physics-based state parameter prediction algorithms are often computationally too expensive. Concurrently, equivalent circuit models (ECM) are often limited due to their poor robustness regarding the highly non-linear dependence of the battery state parameters on the changes of environment and working conditions during a battery's operation. ML and DL approaches like recurrent neural networks, and temporal convolutional neural networks have demonstrated their potential to overcome these problems due to their high adaptability and self-learning ability (Bockrath et al., 2019; AI4DI, 2019). Furthermore, by combining the neural network with a priori knowledge and physical laws, physical guided neural networks can be derived considering physical relationships. Thereby, neural networks are becoming failure-safe, thus ensuring an extremely accurate state parameter prediction. The SOC during a dynamic 30-min city drive is shown in Figure 5. The prediction of each SOC step was executed using a long short-term memory neural network, and the reference SOC was measured using coulomb counting from a high precision current sensor. The results show that neural networks can estimate the SOC accurately, even for a very dynamic driving cycle. Moreover, since coulomb counting needs timeconsuming re-calibrations, neural networks outperform the coulomb counting measurement regarding adaptability and performance.

An application-specific and context-sensitive optimal battery management strategy can be performed with an accurate and real-time estimation of the remaining available energy and power and battery health. According to the battery's state parameter, the

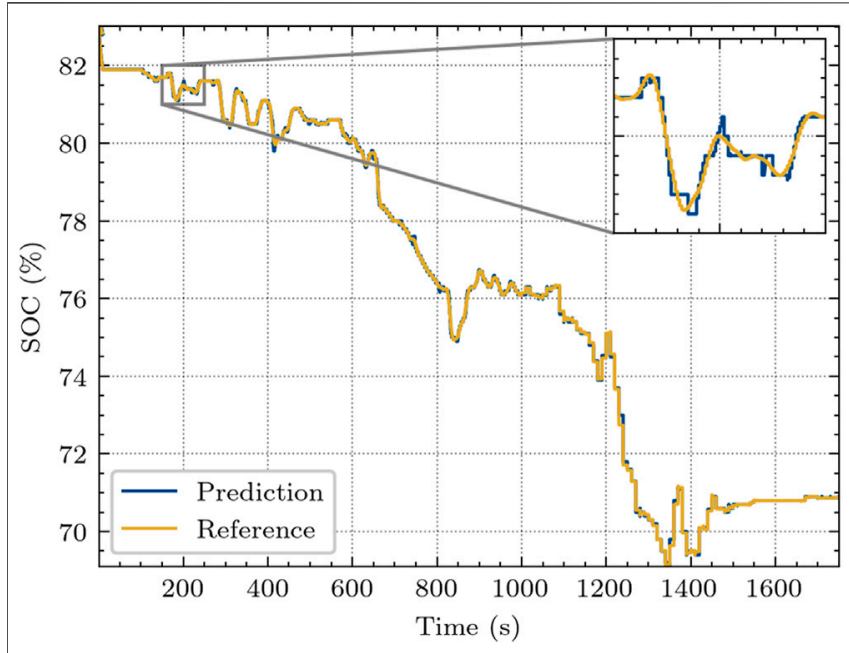

FIGURE 5 | State of Charge (SOC) during a dynamic 30-minute city drive.

routing and traffic, the charging infrastructure, and the environmental conditions, a fully holistic and optimal energy management strategy of an ECAS vehicle can be performed.

The battery's state parameter estimation results are used for an intelligent charging process of the ECAS. Hence, an optimal charging process is executed according to the routing, needed energy, the battery's state parameter, and the maximum requested charging time.

Finally, the BMS provides the battery's state parameter estimations and predictions to the next higher-level control system, the energy management system (EMS). The EMS is a controlling unit of the ECAS vehicle that communicates and exchanges information with the smart grid. ECAS can contribute significantly to improve the electrical grid stability, power quality, balancing, and matching peak power demand in conjunction with the BMS and EMS information and using particle swarm optimization and reinforcement learning approaches. As a result, the fully holistic integration of the ECAS vehicles into smart grids minimizes the cost involved in energy production, storage, distribution, plant maintenance, and operations, while the lifetime, reliability, and safety of the batteries are maximized.

The energy domain includes the interfaces for V2G control and functions. It assures that the interface with the charging infrastructure is safe, convenient, economical, and energy-efficient. An efficient energy management system allows linking large numbers of ECAS vehicles to the grid, opening the way to implementing the IoV paradigm and creating sustainable green mobility ecosystems by integrating different stakeholders (consumers, facility/vehicle fleet managers, and electric utilities).

\section{Powertrain and Vehicle Dynamics Domain}

The domain is responsible for the motion, speed, and dynamics of the ECAS vehicle based on the inputs and commands received from the autonomous driver and cognition domain. The domain functions are adjusted and optimized based on individual 
preferences, vehicle mission, environmental constraints (e.g., road conditions, climate, terrain, etc). The powertrain (e.g., combustion engine, electric engine, hybrid engine) is part of vehicles architecture for internal combustion engine (ICE), hybrid or electric vehicles (Ottella et al., 2010). It has the function of converting the fuel source into power while delivering the power to the road surface. The engine, transmission, driveshaft, axle, and wheels are all part of the mechatronic powertrain system that operates in the harsh environments of all types of roads and weather conditions (e.g., high/low temperatures, vibrations, dust, water, snow, chemicals, etc). Vehicle dynamics address the effect of forces and torques on the motion by utilizing supporting subsystems based on various sensors, microelectromechanical systems (MEMS), and actuator technologies for suspension and steering to ensure stability and a stable drive. The powertrain and vehicle dynamics domain require the use of components that conform to automotive safety integrity level ASIL D to provide the highest level of assurance that the dependent safety goals are sufficient and achieved. ASIL D points to the highest classification of initial hazard (e.g., injury risk, the potential for a severely life-threatening or fatal injury) defined by ISO 26262 (ISO 26262-3, 2018) for safety measures applicable for avoiding unreasonable residual risks (ArchitectECA 2030, 2020). The domain functions are evolving toward adding more intelligent features that require software enablement personalization, updatability/upgradability, intelligent algorithms for sensors/ actuators data fusion. Dynamic links with the perception, autonomous driver and cognition domains are necessary. The domain must ensure cost/form-factor and performance trade-offs depending on the users' requirements and mobility mission profile.

\section{Connectivity Domain}

The connectivity domain covers several functions and integrates both onboard vehicle and external communications. It oversees all the wired and wireless interfaces that connect the vehicle onboard modules and the connections with other vehicles, the Internet, and infrastructure. The connectivity domain securely transfers and exchanges data and information inside and outside the vehicle using internal/external interfaces and communication protocols. That communication technologies include radio, cellular, Wi-Fi, Bluetooth Low Energy (BLE), GPS, V2X, LIN, $\mathrm{CAN}$, and Ethernet. The external interfaces, wireless and cellular, use advanced integrated intelligent antenna modules to increase or reduce interfaces as demanded.

Two technologies for V2X communications are used for ECAS vehicles, unlicensed V2X and cellular V2X. The unlicensed V2X (U-V2X) is based on the IEEE 802.11p/bd technology, such as ITSG5 in Europe, which builds on WLAN and provides an ad-hoc network for the $5.9 \mathrm{GHz}$ ITS band. The incumbent wireless communication technology ITS-G5 is already deployed for safety services in the $5.9 \mathrm{GHz}$ band. In $2019,6,000 \mathrm{~km}$ of roads were already equipped with roadside units (RSUs) facilitating safety using cellular connectivity and ITS-G5. Since March 2020, ITS-G5 supporting road traffic safety is a default feature of the VW Golf 8 and the VW ID models. By the end of 2021, roughly 750000 Golf 8 and IDs are expected to reach the European market. Deployment of so-called day one service for increasing safety increased and facilitated through the deployment of the ITS-
G5 wireless technology (Car 2 Car Communication Consortium, 2021).

Cellular V2X (C-V2X) is an umbrella term that includes LTEV2X for short-range communications (which provides an ad-hoc network for the unlicensed spectrum $5.9 \mathrm{GHz}$ ITS band, similarly to ITS-G5), and the cellular network communications (LTE Uu) for long-range communications.

LTE-V2X has two operation scenarios which are Uu-based and PC5-based. Uu relates to the interface between user equipment (UE) devices and a base station Evolved Node B (eNB). PC5 relates to the one between UEs. Uu-based LTE-V2X utilizes downlink and uplink of LTE networks for communication among UEs. PC5based LTE-V2X employs side links (e.g., direct links between UE devices) for communication among UEs, with and without the support of eNBs. LTE-V2X PC5 interface, has lower latency, while $\mathrm{Uu}$ interface is providing higher throughput using a dedicated band in the licensed spectrum. The licensed spectrum requires that the automotive OEMs have agreements with the operators and licensed proprietors to provide a worldwide service.

PC5-based LTE-V2X is further divided into two modes: mode 3 and mode 4 . In PC5 mode 3, side link resources of each UE are allocated by eNBs, and the UE broadcasts packets using the allocated side link resources. In PC5 mode 4, each UE autonomously selects side link resources and broadcasts packets using selected side link resources. The operation scenario of PC5 mode 4 is like one in U-V2X (DSRC) considering that the channel access mechanisms are fully distributed and both protocols do not require the base stations (Shimizu et al., 2019).

To address advanced use-cases, both technologies specifications are further developed in the standardization working groups of $3 \mathrm{GPP}$ and IEEE 802.11. The IEEE 802.11 next-generation V2X (NGV) task group was established to create a new amendment IEEE $802.11 \mathrm{bd}$. The 4G LTE-V2X technology included in Rel.14, and Rel.15 (composed of $4 \mathrm{G}$ core networks $+5 \mathrm{G}$ base stations) offers basic safety functions with LTE-V2X enabling higher traffic efficiency and assisted driving safety, and supporting other functions like electronic brake light, left turn assistance, automated valet parking (AVP) in a parking lot, and remote-controlled driving. The cellular V2X standard based on $5 \mathrm{G}$ is included in Rel.16 of the standard and is referred to as new radio $5 \mathrm{G} \mathrm{V} 2 \mathrm{X}$ (NR-V2X). NR-V2X is not replacing LTE-V2X but complement it to support the current and future V2X applications with enhanced radio layer technical features and network architecture.

Due to the technological challenges and the need for redundancy in fail-operational autonomous vehicles, global hybrid V2X solutions (e.g., unlicensed + cellular) need to be investigated to provide flexible technology deployments and upgrades (e.g., timing, model, market).

The wireless and cellular part of the connectivity domain requires the use of components that conform to ASIL B and C. In the future, ASIL D may be needed as the ECAS vehicle architectures evolved toward federated vehicle/edge/cloud-centric (Birnie, 2020) based on distributed intelligence at the vehicle and infrastructure level. Robustness, reliability, and low latency communication are becoming a standard requirement for external connectivity for ECAS vehicles that allow the 
coexistence of multiple standard communication protocols and end-to-end security implementation. The onboard gateway and networks coordinate and control the inter domains operations and exchange/share of data and information. The in-vehicle connectivity networks provide the architectural links that integrate the vehicle domains by securing data sharing with required characteristics (e.g., bandwidth, speed, security, reliability, robustness, resilience). The in-vehicle network integrates wired (e.g., CAN, LIN, FlexRay, Ethernet) and wireless (Wi-Fi, BLE, etc.) technologies to securely connect the vehicle domains. The in-vehicle gateway and networks play an essential role in the ECAS vehicle architectures to link the domains and ensure the proper distribution of vehiclegenerated data. The onboard gateway manages the information inside the vehicle, shielding it from external access and external hacking by guarding (firewall) the subsystems and holding them separated from one another to avoid undesired interactions. The onboard gateway secures that each vehicle domain's large amounts of data are routed efficiently and reliably and that the vehicle safety-critical systems are shielded from other systems' actions. The onboard gateway and networks part of the connectivity domain requires the use of components that conform to ASIL D, provide connectivity robustness, low electromagnetic emissions, the coexistence of secured multistandard wired and wireless protocols. The Automotive SERDES Alliance (ASA, 2020) was created in 2019, and it is rapidly growing, counting among others NXP, BMW, and Continental as its founding members. The ASA standard specifies profiles for secure, highly reliable, automotive invehicle bridged IEEE 802.3 Ethernet networks with deterministic latency, based on IEEE 802.1 Time-Sensitive Networking (TSN) and security standards. It supports the entire range of in-vehicle applications, including those requiring security, high availability, reliability, maintainability, bounded latency, and form the base for future in-vehicle networking.

\section{Perception Domain}

The domain includes the HW/SW modules, components, and intelligent algorithms use to sense and interpret the environmental observations produced by various perception devices (cameras, radars, LiDARs, ultrasound sensors) as presented in Figure $\mathbf{6}$ and features for location and positioning. The domain enables the localization/positioning, perception, and planning functions for ECAS vehicles. Perception fuses the data from the perception sensors into a representation of dynamic objects and static features. By matching the model to elements of a full pre-generated environment map, intelligent algorithms are applied to compute the autonomous vehicle's current position and orientation accurately. The vehicle's location/position and orientation linked with the map and fused object information coming from perception allows the planning component to produce a sequence of intelligent movement operations. The perception domain is tightly connected to the autonomous driver and the cognition domain, which combines the

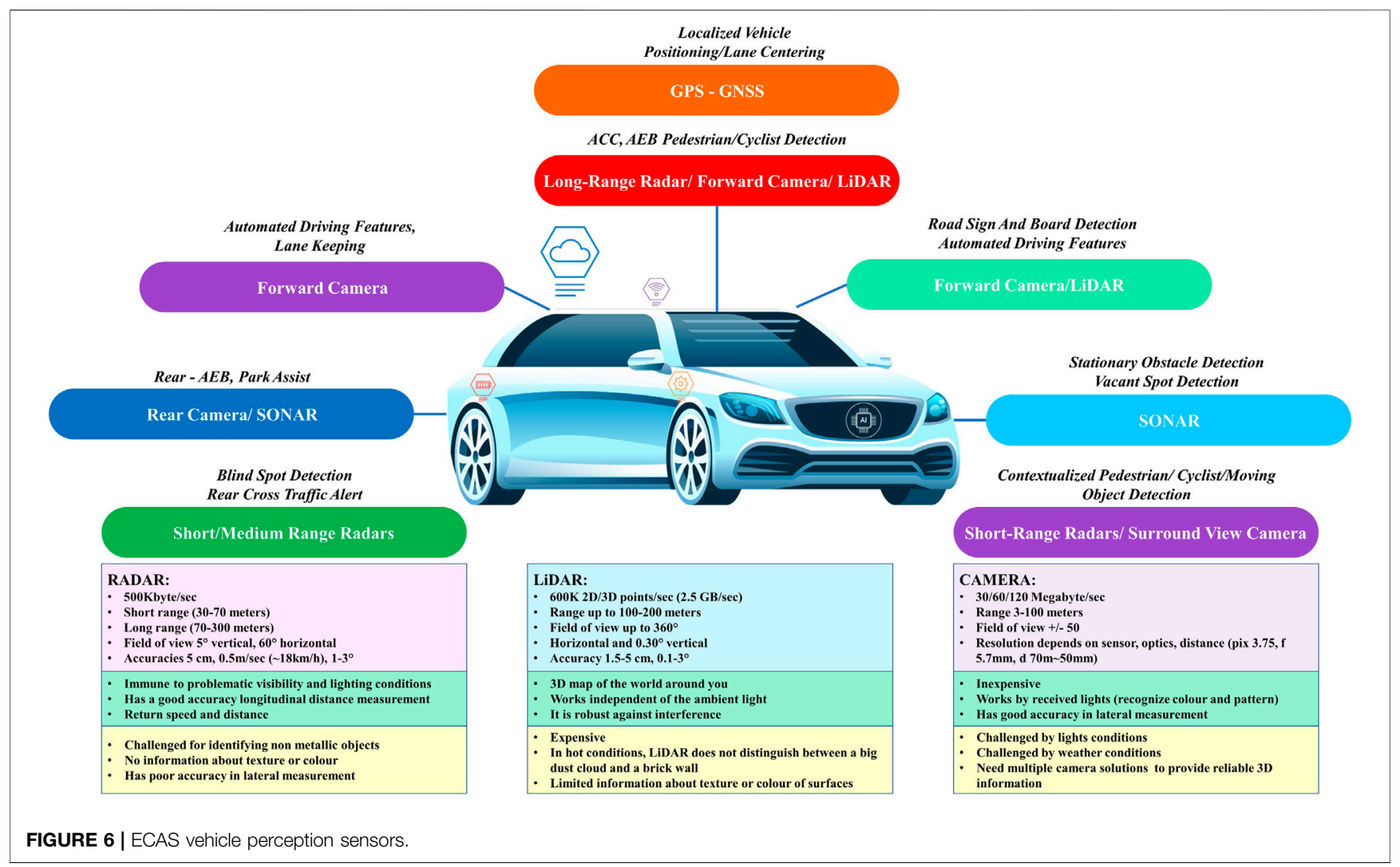


information related to the situational awareness, processes the predicted behavior of other traffic participants, and considers the potential reciprocal interaction in a specific context scenario. AIbased techniques are used for implementing the perception functions in ECAS vehicles. The perception domain requires the use of components that conform to ASIL D, provide robust and resilient pattern/object recognition, a high level of data fusion, and ensure several AI-based technologies coexistence.

Fail-operational safety and redundancy are achieved by using redundant sensors and AI-based algorithms (Mohseni et al., 2020) for safety-critical functions. The redundancy is optimized using sensor fusion for autonomous vehicle functions. Radar, camera, and other perception sensors are used in combination to provide safe, secure perception functions, with solid-state Light Detection and Ranging (LiDAR) and camera integration offering optimal solutions for long-term solutions. The strategies for aggregating the information from various perception sensors are supporting different approaches with solutions where the sensors are connected to several domain controllers as the perception are intelligent and highly integrated (sensing, processing, AI-based algorithms, etc.), and transfer only critical information to the centralized controller to reduce the complexity of the overall vehicle architecture. Other approaches use centralized control units to perform a real-time fusion of raw data collected from various perception sensors to enable real-time performance and cost reduction of the overall system.

\section{Autonomous Driver and Cognition Domain}

The autonomous driver and cognition domain provides the planning, computing, cognitive, and decision-making autonomous driving functions for ECAS vehicles. It integrates sensing fusion and capabilities of perception and connectivity domains and applies cognitive processing, decision-making, and safeguards to ensure proper vehicle operation in various conditions and context scenarios. The cognitive abilities include mission analysis, path planning, situational assessments, sensor fusion, safety-related algorithms, and multi-scenarios decision-making. The actions decided by the autonomous driver and cognition domain are applied to the powertrain and vehicle dynamics domain as presented in Figure 7 to control the direction steering and to provide acceleration and braking. The autonomous driver and cognition domain is implemented using high-performance embedded edge computing and AI-based technologies (Han et al., 2019). It has learning capabilities that allow the domain to take on new knowledge gained from driving and traffic experiences through a training/learning process using edge/cloud computing infrastructure. The ECAS vehicle training/ learning process is done continuously by connecting to the edge/cloud computing infrastructure and uploading data sets accumulated during missions. The vehicle data sets can be aggregated with data from other vehicles and can be used to optimize driving algorithms. The training/ learning process can be done when the vehicle is not used, parked, or stored in a garage. The autonomous driver and cognition domain requires the use of components that conform to ASIL D, the continuous homologation/ certification and qualification of components/functions based on standardized procedures, fail-operational capabilities, and dynamic learnability.

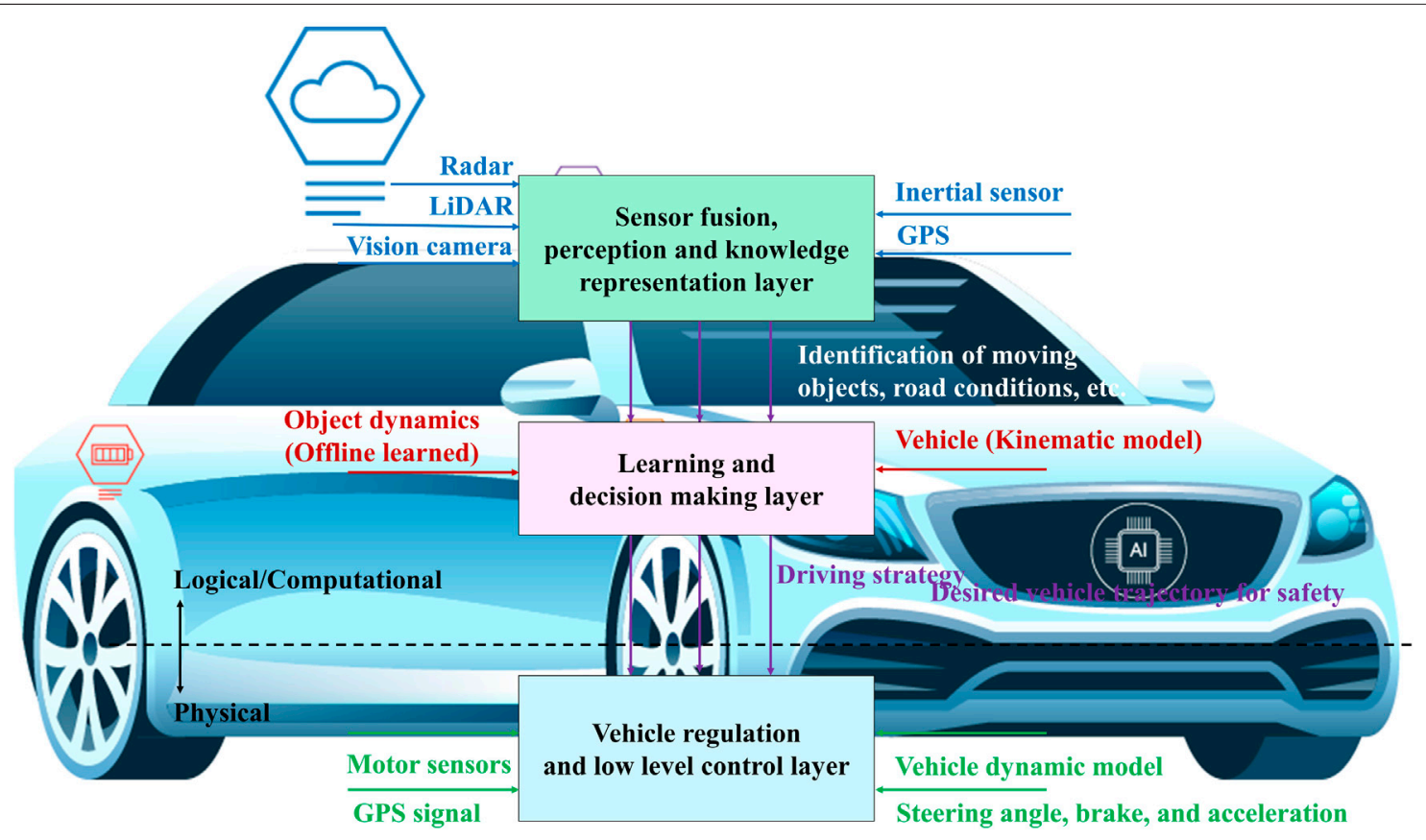

FIGURE 7 | Layered Al-based processing and cognition in ECAS vehicles. 


\section{Body and Comfort Domain}

The body and comfort domain supports the passenger's essential functions and observes behavior to learn preferences and combine them with safety requirements. The domain controls the passive safety mechanisms (seatbelts), access mechanisms (door locks), window blinds. It can allocate the vehicle settings for various passengers by defining the seat position adjusting the climate control temperature, and the illumination intensity. The domain oversees the window controls and seat adjusters and provides the management and modification of the SW/HW components for proper operations. The sensors/actuators and controller functions are optimized to create ambient experiences for passengers that increase safety and match personal preferences. Interior/exterior lighting, headlights are automatically customized for weather conditions, or the presence of oncoming traffic providing areas for passengers to sleep or read or watch a video, with dashboard settings adjusted automatically based on the period of a day or the type of passengers that are in the vehicle. The body and comfort domain demands the use of components that conform to ASIL B, provide updateability/upgradability functions, maintainability, energy efficiency, and AI-based technologies to increase real-time monitoring and learnability capabilities.

\section{Vehicle Experience Domain}

The vehicle experience domain evolved from the infotainment vehicle domain to provide the vehicle capabilities to offer all passengers the requested onboard well-being, comfort, entertainment, and productivity. It allows seamless access to digital content and enables passengers to create and manipulate digital and virtual content. The domain integrates advanced human-machine interfaces (HMIs) to support voice commands, gestures, augmented reality, and a progressive learning environment that adjusts to passenger's preferences. The vehicle experience domain requires the use of components that conform to ASIL B, provide software/features OTA updateability/upgradability functions, augmented/virtual reality functions, maintainability, and AI-based technologies advanced HMI, real-time monitoring, and learnability capabilities.

\section{AUTOMOTIVE INTELLIGENCE DESIGN ISSUES AND AI TECHNIQUES AND METHODS FOR THE IMPLEMENTATION OF THE ECAS VEHICLES}

\section{Operational Design Domain}

Safe autonomous driving depends on setting the exhaustive account of overlapping conditions an ECAS vehicle might meet, termed the operational design domain (ODD). Safety standards and metrics for ECAS vehicles are under development, and an alignment between legacy standards and the new requirements and technologies applied to new vehicles is underway. The ODD poses many challenges since there is a need to define all possible overlapping conditions, use cases, restrictions, and scenarios that an $\mathrm{AV}$ might encounter, even the most obscure corner cases. ODD factors include the time of day, weather, terrain, road conditions/features, and context scenery. There are factors that ECAS vehicles might encounter and impact the traffic conditions and driving decisions. ODD characteristics add glare/light/flash, outdated mapping detail, overhanging vegetation, falling objects, downed power lines, toll booths, water-filled potholes, icing, social norms, uncooperative people, delivery robots, and common human rule-breaking. The use of AI-based techniques and methods brings new challenges for establishing ODD-based autonomous vehicle safety standards considering the need to reduce the residual risk and implementing fail-operational procedures. Developing standards for ECAS vehicles requires a holistic approach that considers defining virtual validation and repeatable testing conditions with a baseline of requirements and conditions to evaluate the vehicles based on equal comparisons of ECAS systems throughout the world. Applying the ODDs to the certification and homologation processes needs to be done in steps, initially in more accessible and controlled driving environments, and then moved gradually in increasingly complex locations and conditions.

The ODDs require being agnostic to the technology used by various ECAS vehicles for the perception, propulsion, sensor fusion, or cognition algorithms to achieve safety-performance benchmarks. The search for equal comparisons and measurements is challenging due to several groups of stakeholders trying to establish standards for delivering safety and support the deployment of the ECAS technology.

\section{Integrated Intelligent Hardware and Sensor Fusion Functions}

The hardware must be analyzed in detail to get a trustable ECAS system. All sensors must be able to check themselves, and all the sensor networks must be based on reliable hardware and trustable data analysis. Hence even the use of reliable components will not avoid any faults. The monitoring of the HW/SW components and their functions is crucial and brings decisive added value. The number of signals will be very high. To evaluate the right status, new AI bases data analysis methodologies are necessary. This comprises the continuous monitoring of component properties as well as parameter shifts. Self-learning methods and classification according to the system behavior and the final decision making will be carried out in parallel to continuous data analysis. This means changes and deviations in functionality will be detectable. This opens the path from a simple pass/fail decision to a detailed system assessment and intelligent decision-making.

As already described, the reliability of the powertrain hardware is crucial. It is essential to find practical and effective solutions for trustable powertrain systems. Current sensor-based systems are typically modularly designed with different sensors, connected within vehicles and externally. Some of these systems include the human as a control unit, which reduces the amount of special sensor monitoring. If the sensor information is not accurate enough or a significant defect 
is detected, the driver can often compensate for such an issue. Humans bring the system into a more secure state, e.g., lowering the speed of a vehicle.

Here the primary intelligence is located at the control place, and the driver is skilled to handle such situations. New SAE Level 5 systems will no longer need human driving skills, but reliability and trustability requirements will increase significantly.

For a reliable powertrain, continuous health monitoring of the power electrics and the electric motor is necessary. For health monitoring of the power electronics, the temperature is the critical parameter. Today many approaches for determining the power device temperature exist. The device temperature depends on the electrical load and pre-history of operation. Most methods are based on measurements of temperaturesensitive electrical parameters or dedicated sensors. The temperature-sensitive electrical parameters are, in general, not easy to measure as the required high accuracy and time resolution, which is challenging to implement in powertrain drive inverters. From temperature measurements, the remaining lifetime can only be estimated because the lifetime models are exponential laws. Any inaccuracy in measurements results in high errors at conventional lifetime prediction. For autonomous applications, the lifetime models must be much more precise to avoid unplanned failures during operation or waste of remaining lifetime.

Instead of using the current physics-of-failure $(\mathrm{PoF})$ approach, an alternative data-driven approach should be implemented, such that the correlation with degradation is found in trends of actual device function - often caused by a blend of effects. AI and deep learning algorithms can extract early warning signals. However, these techniques suffer from the extremely long time series that require significant computing power. Furthermore, they are often based on assumptions about the regularity of time sampling, which cannot be guaranteed in real applications. Combining both approaches, PoF and DD, through meta-models can lead to routines that suffice with edge computing power. Then, health monitoring can even be used to adjust machine performance in real-time according to functional requirements and health status. Instead of blindly continuing at $100 \%$ power, leading to failure in $5 \mathrm{~h}$, an intelligent reduction to say $80 \%$ can extend the remaining useful life (RUL) to $50-100 \mathrm{~h}$, long enough for maintenance to occur at a convenient time. This would be a perfect feature for autonomous systems.

A vast amount of temperature data is collected in different modes of operation. With AI, it is possible to detect patterns of increasing fatigue. Therefore, self-learning systems are crucial for a trustable lifetime prediction avoiding any unplanned failures during operation.

The development of technologies for ECAS vehicles in the energy domain, including the power train's electrification and the integration with the smart grids, are catalysts to reducing $\mathrm{CO}_{2}$ emissions and more intelligent transportation systems. Rigas et al. (2015) provide an overview of AI techniques for EVs and the systems that manage fleets of EVs and give a literature overview to identify the commonalities and fundamental differences in the approaches. The paper addresses solutions EVs use to augment the grid with the ability to store energy and bi-directionally exchange energy, optimize energy from intermittent renewable energy sources and let users refill their vehicles in a variety of locations. The different approaches consider charging EV batteries and using AI-based algorithms that minimize costs and increase battery life while avoiding users from being stranded. Sun et al. (2020) propose an EV charging behavior prediction design based on hybrid AI to identify targeted EVs and predict the charging behavior. Simulation results confirm that the proposed prediction scheme outperforms the existing EV charging behavior prediction methods in forecasting accuracy and scheduling efficiency.

The development of new power train solutions based on distributed powertrain architectures requires AI algorithms to optimize the ECAS vehicle propulsion system. The ECAS vehicle architectures must provide redundant power networks using $\mathrm{x}$-by-wire functions through fail-safe/fail-operational power supply/energy functionality without additional storage units. The power topologies are optimized (e.g., star) to ensure the energy paths to safety-critical loads and optimal wiring circuits. The topologies integrate modular power distribution units that allow load monitoring, fast failure detection, system response, and actions on the driving system.

The electric motors are critical components of the powertrain. The design of the motors for ECAS vehicles demands, considering the requirement for high power and torque densities, a wide range of speed at a constant power operation (e.g., 3-4 times the base speed), high starting torque with the ability of a hill-climbing, a high-level of reliability, high robustness, intermittent overload ability for overtaking, reduced torque ripple and minimum noise, advanced AI-based control methods, and an affordable cost. Different types of motors such as DC drives, induction motors (IM), synchronous (SR) machines, permanent magnet (PM) drives are used in EVs. An overview of the state-of-the-art solutions of electric motors for EVs is given in Alamoudi et al. (2019), with an overview of automotive electric propulsion systems with reduced or no permanent magnets presented in Boldea et al. (2014) and a review of synchronous reluctance motor-drive advancements provided by Heidari et al. (2021).

\section{Intelligent Connectivity}

The IEEE has specified the 802.11 protocol to provide the MAC for WLAN communication in different frequencies. IEEE 802.11 enables easy Internet connections allowing the WLAN benefits such as the more straightforward wireless station deployment. The IEEE 802.11 protocol is used for the unlicensed V2X implementation. Safety and V2X need to be affordable considering all the cost variables such as service running cost, technology access fees, and licensing costs. The unlicensed V2X solutions based on the IEEE 802.11p have proven real-life performance, relative low latency $<10 \mathrm{~ms}$, ranges $>1.4 \mathrm{~km}$ in line-of-sight (LOS) and $>1 \mathrm{~km}$ non-lineof-sight (NLOS) ( $\mathrm{Gu}$ and Moerman, 2020), standalone deployment without dependence on cellular for safety applications, stable performance in congestion (PRR, latency), and provides full compatibility with nextgeneration IEEE $802.11 \mathrm{bd}$ standard by design (Naik et al., 
2019). IEEE 802.11p is used for ETSI ITS-G5, and massive rollout of vehicle is ongoing (Car 2 Car Communication Consortium, 2021). The IEEE 802.11bd standard is specified to be backward compatible with IEEE $802.11 \mathrm{p}$ and provide latencies below 2-3 ms.

The 3GPP GPP3 (2018) has specified the cellular C-V2X in different releases (Rel.14/15/16). C-V2X technology supports additional operation modes and will provide increased throughput in the new 5G NR version adopted in Rel.16. The $\mathrm{C}-\mathrm{V} 2 \mathrm{X}$ will provide increased environmental perception to enable sensor-data sharing among vehicles and infrastructures. It could enhance the automated driving control, allowing the vehicle's cooperation by the perception and the control subsystems of $5 \mathrm{G}$.

The field and interoperability tests for the C-V2X technology just began. The technology, today, offers a latency of 20-100 ms, needs of GNSS clock (PC5 Mode 4) or cellular (PC5 Mode 3 or Uu) for synchronization at $\mathrm{PHY} / \mathrm{MAC}$ level and can have performance degradation in high vehicle density cases ( $\mathrm{Gu}$ and Moerman, 2020). Several technical limitations related to the half-duplex and near-far problem are identified. The LTE-V2X and IEEE 802.11p were compared in Connected Corridor for Driving Automation (CONCORDA) project considering the technology from a PHY and MAC perspective, shedding light on important technology choices in both approaches (Moerman et al., 2020). In addition, the authors investigated the impact that technologies have on each other in terms of quality of service (QoS) and possible mitigation measures to broker the differences toward a result that still provides interoperability and partially mitigates the QoS problems. These results arise from research and participation in the ETSI working groups tasked with these questions.

In a comparison between both technologies, in general, uncongested conditions show similar performance behavior between LTE-V2X and IEEE802.11p under comparable system settings. However, in specific detailed traffic situations, behavior is expected to be different. In congested conditions, IEEE802.11p appears to have an advantage in packet-delivery rate vs. range and associated system latency. It varies smoothly with the payload size, as in LTE-V2X Rel.14, having multiple users in the same $1 \mathrm{~ms}$ time slot reduces the performance. This is partly due to some weaker design features in the Rel.14 implementation, which are acknowledged and addressed in updates in Rel.15 or 5G-NR V2X Rel.16+. However, these updates do not help for safety applications, as REl.14 is defined as the version to use for the safety use-cases, while Rel.15 and Rel.16 updates are used only for new advanced use cases (Moerman et al., 2020).

A system-level comparison of the performance of U-V2X and LTE-V2X when transmitting periodic and aperiodic messages with constant or variable size is presented in (Molina-Masegosa et al., 2020).

U-V2X outperforms LTE-V2X when the channel load increases, even if it has a lower physical layer performance. The gains obtained are present even with periodic messages of constant size, and they significantly augment when messages are aperiodic, and their size varies.

LTE-V2X can achieve better performance than U-V2X under low channel loads if it has a better physical layer performance.
Under low channel load levels, the MAC has a lower impact on the performance than the physical layer. The challenges for the LTE-V2X MAC exist due to the design of the standard and the impact depends on the characteristics of the messages to be transmitted. The dependency is less present in U-V2V (IEEE 802.11p) that implements a simpler and more flexible MAC (Molina-Masegosa et al., 2020).

The performance comparison of U-V2X (DSRC) and LTEV2X PC5 mode 4 defined by Rel.14 is performed in Shimizu et al. (2019) using congestion control mechanisms in freeway scenarios for several vehicle densities. The simulation results point that in a lower vehicle density case, the two technologies are comparable or LTE-V2X provides a longer V2V communication range, whereas in a high vehicle density case, U-V2X (DSRC) delivers superior performance than LTE-V2X PC5 mode 4. U-V2X (DSRC) achieves a significantly shorter end-to-end latency. in both high and low vehicle density scenarios. The analysis is based on one configuration, focusing on freeway scenarios. As there are a lot of configurable parameters in LTE-V2X PC5 mode 4 and other scenarios, it requires more thorough evaluation and analysis for different configurations and scenarios (e.g., especially congested scenarios) to make LTE-V2X PC5 mode 4 mature (Shimizu et al., 2019).

C-V2X releases are not compatible with each other as Rel.14/ Rel.15 and Rel.16 operate in different frequency bands, and the V2X components must support multiple releases to keep compatibility with older devices.

The unlicensed U-V2X and cellular C-V2X technologies operate in the $5.9 \mathrm{GHz}$ band, with $\mathrm{C}-\mathrm{V} 2 \mathrm{X}$ operating in the cellular operator's licensed carrier. The need for multi-radio interoperability between the currently available radio technologies (U-V2X and C-V2X/LTE-Uu). The requirement to reinforce the primary security system through functions that detect vehicles misbehaviors due to external attacks (GPS spoofing) or physical attacks as tampering attempts to access the vehicle's cryptographic material is highlighted by Casademont et al. (2020).

The LTE-V2X cellular technology can provide up to around $1,400 \mathrm{~m}$ of short-to-medium-range connectivity with GNSS timing reference and a latency of $20-100 \mathrm{~ms}$. Latencies in the LTE-V2X short-to-medium range technology can be considered about $50 \mathrm{~ms}$ or more (the LTE-V2X radio-technology alone provides lower latency, but combined with the protocol, varying message sizes, etc. becomes of the order of tens of ms magnitude). In synchronous systems like LTE-V2X, the signal of all users must arrive time-aligned to the receiver to prevent intersymbol interference between consecutive OFDM symbols. Achievable communication range distance of LTE-V2X based on timing reference source (e.g., GNSS, eNB) is limited, and beyond that, a receiver cannot detect messages from far users (Filippi et al., 2018). LTE-V2X allows transmission data rates up to $75 \mathrm{Mbps}$ for uplink and up to $300 \mathrm{Mbps}$ for downlink (Arena and Pau, 2019). The C-V2X technology is moderately suitable for $\mathrm{V} 2 \mathrm{~V}$ applications and increased suitability for V2I at a high communication cost. The U-V2X technology provides $300-1,000 \mathrm{~m}$ of short-to-medium-range connectivity and below $10 \mathrm{~ms}$ latency, enabling a rate of up to $27 \mathrm{Mbps}$ for data transmission (Storck and Duarte-Figueiredo, 2020). The U-V2X 
TABLE 2 | Vehicular communication technologies and key performance indicators.

\begin{tabular}{|c|c|c|c|c|}
\hline \multirow[t]{2}{*}{ Application requirements } & \multirow{2}{*}{$\frac{5 G \text { NR V2X }}{\text { 3GPP Rel.16 }}$} & \multirow{2}{*}{$\frac{4 G \text { LTE-V2X }}{3 \text { GPP Rel.14 and } 15}$} & \multirow[t]{2}{*}{ IEEE 802.11bd } & \multirow[t]{2}{*}{ IEEE 802.11p } \\
\hline & & & & \\
\hline Radio bands & $5.9 \mathrm{GHz}$ & $5.9 \mathrm{GHz}$ & 5.9 and $60 \mathrm{GHz}$ & $5.9 \mathrm{GHz}$ \\
\hline Waveform & OFDM/DFTs OFDM & SCFDMA & OFDM & OFDM \\
\hline Modulation & QPSK, 16-QAM, 64-QAM & $\begin{array}{l}\text { Rel. 14: QPSK, 16-QAM } \\
\text { Rel. 15: 64-QAM }\end{array}$ & $\begin{array}{l}\text { BPSK, QPSK, 16-QAM, } \\
\text { 64-QAM, 256-QAM }\end{array}$ & $\begin{array}{l}\text { BPSK, QPSK, 16-QAM } \\
\text { 64-QAM }\end{array}$ \\
\hline Communication & Broadcast, Groupcast & Broadcast & Broadcast & Broadcast \\
\hline PHY layer & Unicast & & & \\
\hline Bandwidth & $\begin{array}{l}\text { sub-6 GHz: }<100 \mathrm{MHz} \\
\text { mmWave: }<400 \mathrm{MHz}\end{array}$ & $1.4 / 5 / 10 / 20 \mathrm{MHz}$ & 10 and $20 \mathrm{MHz}$ & $10 \mathrm{MHz}$ \\
\hline Data rate & 1 Gbps & $28.8 \mathrm{Mbit} / \mathrm{s}$ & 3-54 Mb/s & 3-27 Mb/s \\
\hline Latency & $<10 \mathrm{~ms}$ & $<100 \mathrm{~ms}$ & $<2-3 \mathrm{~ms}$ & $<10 \mathrm{~ms}$ \\
\hline Mobility & Up to $500 \mathrm{~km} / \mathrm{h}$ & Up to $140 \mathrm{~km} / \mathrm{h}$ & Up to $500 \mathrm{~km} / \mathrm{h}$ & Up to $250 \mathrm{~km} / \mathrm{h}$ \\
\hline Artificial intelligence & No & No & No & No \\
\hline Satellite integration & No & No & No & No \\
\hline
\end{tabular}

is suitable for $\mathrm{V} 2 \mathrm{~V}$ applications and moderately suitable for V2I applications, but it requires visibility. According to Selvanesan et al. (2018) and Frost Sullivan (2017), the technology is not ideal for multimedia features requiring high bandwidth. An overview of vehicular communication technologies and their key performance indicators (KPI) is provided in Table 2.

AI techniques are used in the connectivity domain of the ECAS systems to protect against cybersecurity attacks and improve the vehicle's overall security. A survey of topics related to the secure vehicular network-applied ML approach is presented in Tang et al. (2020). The ML techniques are classified for the vehicular network as communication, networking, and security perspective emphasizing the future of vehicle network that include $6 \mathrm{G}$ and AI techniques. Usman et al. (2020) investigated ML techniques' introduction to identifying relay attacks against PKES systems. The decision tree, support vector machine, and k-nearest neighbor's methods were compared for the experiment utilizing a three-month log of the PKES system. Song et al. (2020) offered an IDS based on a deep convolutional neural network to protect the CAN bus. Different solutions were considered and compared, including various ML algorithms (e.g., LSTM, artificial neural network, support vector machine, k-nearest neighbors, naive Bayes, and decision trees), for four categories of attacks (e.g., Denial of Service-DoS, Fuzzy, Gear Spoofing, Revolutions per Minute-RPM Spoofing). The experiments were performed on the proposed model with these four categories of message injection attacks.

The AI-based technologies used in the design of ECAS vehicles require that the validation and testing procedures must include additional methods to ensure that each performance metric is supported by a clear explanation (i.e., present the safety risk/ crash scenario that each metric is designed to address and how the metric can address that risk/scenario). Besides, new methodologies for virtual validation (Koopman and Wagner, 2016) and simulation must be considered.

Connectivity technologies are an integrated part of the connectivity domain architecture, including several communication technologies based on IoT, ITS, VANET, wireless, wired, cellular, and satellite technologies. The intercommunications between the vehicle and the environment use several channels and different communication protocols. AI techniques are used to optimize the communications and services provided through these channels. A list of these channels (AUTOPILOT, 2017) is presented in below.

- Vehicle-to-Body (V2B) - Internal information transmission.

- Vehicle-to-Cloud/Edge (V2C) - Wireless communication between vehicle and the cloud or edge computing centers.

- Vehicle-to-Device (V2D) - Short-range wireless communication between the vehicle and devices (IoT) either inside or outside the vehicle.

- Vehicle-to-Grid (V2G) - Wired/wireless communication between electric vehicle and the charging station/power grid.

- Vehicle-to-Home (V2H) - Wireless communication between vehicle and a fixed or temporarily home (e.g., real-time routing).

- Vehicle-to-Infrastructure (V2I) - Wireless communication between vehicle and roadside units (RSUs), external communication devices/ platforms and traffic facilities.

- Vehicle-to-Maintenance (V2M) - Wireless between the vehicle and the vehicle condition responsible (e.g., automotive manufacturer, repair shop), including vehicle condition monitoring, predictive maintenance notification or alerts.

- Vehicle-to-Network (V2N) - Wireless communication between vehicle and cellular networks, (e.g., value-added services).

- Vehicle-to-Owner (V2O) - Wireless communication between vehicles and its owner (e.g., car rental, fleet management, freight tracking).

- Vehicle-to-Pedestrian (V2P) - Wireless communication between vehicle and vulnerable road users (VRUs) (e.g., safety-related services).

- Vehicle-to-Users (V2U) - Wired/wireless exchange of information between the vehicle and its current user including situational information.

- Vehicle-to-Vehicle (V2V) - Communication between mobile vehicles (e.g., surrounding vehicles position). 
The inclusion of non-V2X information received (LiDAR, radar, camera, ultrasonic, inertial sensors) in the development and validation process for performance and test metrics to address the fail-operational operation for autonomous vehicles is required. Several V2X and non-V2X systems must coexist on a vehicle and validation, and test procedures must cover all of them. C-V2X and U-V2X are not compatible with each other and when certain vehicles use U-V2X and others use C-V2X, these vehicles are not able to communicate with each other, so seamless communications for ECAS vehicles cannot be implemented yet.

The co-existence problems derive from primary differences between the technologies such as asynchronous vs. synchronous channel access networks, differences in the channel access scheme: reservations vs. listen-before-talk and differences in sampling rate, modulation encoding/decoding, and modulation of the signal. The co-existence issues are studied under ETSI standardization working groups on same-channel co-existence, split-channel co-existence, harmonized radio standard, and multi-channel operation. A close technical look at U-V2X (802.11p) and LTE-V2X for V2X applications further confirms their complementary nature (Filippi et al., 2018). As result, for now the V2X technology differences resulted in no common ways forward. Therefore, the discussions on conceptual and inventory levels will continue in the future (Moerman et al., 2020).

The end-to-end security is vital for adopting ECAS vehicles and applications and must be addressed at all vehicle architecture layers. The new 5G architectures used for V2X connectivity (Automotive Association 5GAA, 2016) provide the communication layer's functions and components designed to close security gaps from previous cellular networks. The pervasive nature of $5 \mathrm{G}$ introduces new security challenges outside the traditional space. Security considerations need to examine various aspects of the software, virtualization, automation, orchestration, and Radio Access Network (RAN) considerations. Zero-Trust, security and several other techniques are discussed to mitigate the threats, and various recommendations are proposed for security in (GPP3, 2019; 5G Americas, 2020).

In-vehicle connectivity is evolving toward the automotive Ethernet as a high-speed backbone. Automotive Ethernet invehicle network provides higher bandwidth (1 Gbps and beyond), improved security, load balancing controlled by Ethernet switches, low-latency (e.g., use of the time-sensitive network TSN to implement low-latency communication), fewer ECUs and cables. The new E/E ECAS vehicle architectures will provide solutions for the cohabitation of heterogeneous networks, including CAN, FlexRay, LIN. The in-vehicle networks rely on high-data-rate switched Ethernet to ensure reliable inter-domain communication, with topologies (e.g., ring) that offer additional redundancy by duplicating links between switching elements. The switched Ethernet networks will be enhanced by using Ethernet audio-video bridging (AVB) and TSN for real-time components and computing units. In developing the vehicle architectures for ECAS vehicles, the Ethernet is used as the backbone supporting different bus types, including existing bus systems linked to the central gateway. Several architectures use switches to allow connections to any number of devices and provide flexibility in the architectural design of the ECAS vehicles ensuring interoperability for integration with external networks, highbandwidth, reduced cost, and less cabling.

ECAS vehicles must have seamless connectivity provided by wireless, cellular, and satellite technologies that meet safety requirements, fail-operational behavior, and enhance reliability through inherent redundancy. The development of cellular (e.g., 5G, 6G, and beyond) and other wireless technologies [e.g., Wi-Fi 6 , Wi-Fi 7, wireless sensor networks (WSNs), etc.] provide costefficient, robust, and redundant solutions for developing horizontal multi-service networks for ECAS vehicles applications and services.

\section{Al Based Techniques}

Amparore et al. (2014) describe a concept for implementing a virtual driver, which adopts a human cognitive framework as a reference and follows an AI approach. The virtual driver solution proposed utilizes probabilistic techniques (e.g., Markov Decision Process - MDP) and is based on a Cognitive Architecture for Safety-Critical Task Simulation (CASCaS) architecture developed by Lüdtke et al. (2009) and Lüdtke (2014). A key concept underlying the CASCaS architecture is the theory of behavior levels, which distinguishes tasks concerning the requirements on attentional control conditioned on the prior experience (e.g., autonomous behavior by acting without in daily operations, associative behavior by acting in typical situations, and cognitive behavior by acting with new behavior in unknown situations). The model also provides parallelism between the autonomous and associative layers, simulating the human ability to manage tasks simultaneously.

A comprehensive survey of DL applications for object detection and scene perception in autonomous vehicles applicable to the perception domain is provided by Gupta et al. (2021). The paper examines the theory underlying self-driving vehicles from the DL perspective and current implementations, followed by critical evaluations. It introduces DL and computer vision, followed by an overview of AGI, a classification of existing DL libraries and their role and significance, discussing different techniques that approach the image perception issues in real-time driving, and critically assessing implementations tests carried on autonomous vehicles. The findings and practices are summarized to correlate various techniques and the applicability, scalability, and feasibility of DL for autonomous vehicles. The article highlights future work required to advance technologies for perception and object detection to navigate safely. Driving scene understanding and segmentation are integrated with temporal propagation of information to understand both space and time using various DL architectures (e.g., recurrent neural networks - RNNs) to generate automatic captioning of images, localizations, and detection. In several cases, AI-based systems used for autonomous vehicles decide based on a cost/reward function and execute a maneuver correlated with the lowest cost/highest reward. If an unsafe/dangerous maneuver is the lowest cost option, the autonomous vehicle will execute that maneuver anyhow, which requires reviewing the cost/reward strategy in vehicular AI. 


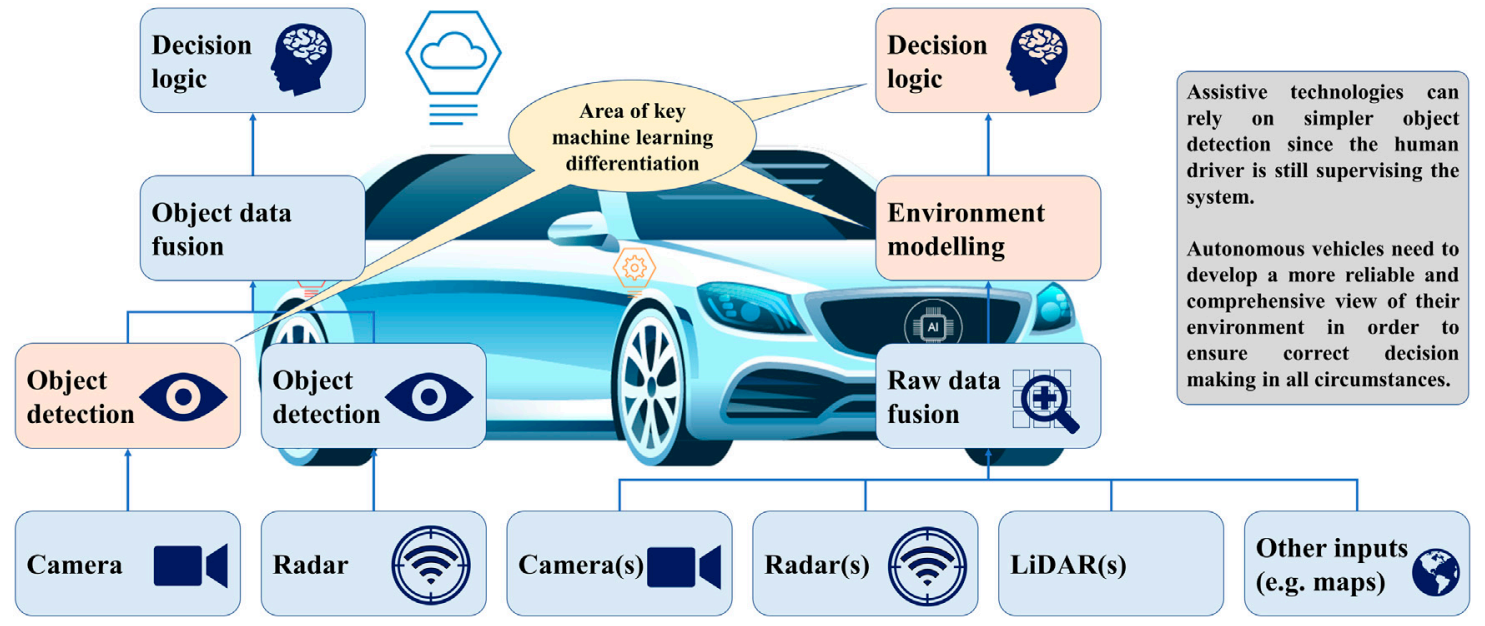

FIGURE 8 | Information fusion approaches in ECAS vehicles.

A comparison of the ML differentiation using object and raw information fusion is presented in Figure 8.

A model for obstacle avoidance in autonomous vehicles by integrating swarm intelligence with pre-existing conventional technologies is presented by Hashim et al. (2019). The concept is establishing bi-directional communication of sensory data between the various vehicles that form a network and overcome the limitations of an autonomous vehicle's perception sensors.

Simulations results performed by Li L. et al. (2018) showed that autonomous vehicles equipped with V2X connectivity and conveying traffic information (e.g., speed advisory or signal timing) using swarm algorithms could fast and efficiently form a stable platoon within $30 \mathrm{~s}$.

The ultimate objective for the entire system is to take measurements from agent's sensors, such as cameras, LiDARs, or RaDARs, and estimate a world model, its perception system. As is typical in $3 \mathrm{D}$ environment sensing, designing a comprehensive "hand-crafted" model is not feasible; instead, the model is learned from training data, using machine learning, such as semantic segmentation (Meletis and Dubbelman, 2018), part-level panoptic segmentation (de Geus et al., 2020), human pose estimation (Cao et al., 2021), action recognition (Li Z. et al., 2018), and depth and flow (Zou et al., 2018).

Current deep learning approaches are compared to human perceptual-cognitive capabilities, still limited in generalization, regularization, completeness, and introspection. These limitations prevent autonomous mobile agents or vehicles from achieving human-like perception levels and, therefore, also human-like decentralized decisions and actions.

According to Bose (2019) operating under changing in-field data patterns and under run-time operations is difficult in current AI interference engines. Precipitous loss of in-field inferential accuracy can lead to catastrophic errors in decisions. Therefore, it is important to focus on resilience mechanism for in-field data changes and inconsistencies for safety-critical systems in run-time operations.

\section{Safety and Security}

Safety is an obligatory characteristic of autonomous vehicles, and AI can impact vehicle's safety. The adoption of novel AI-based technologies in business-relevant safety-critical domains (e.g., an autonomous vehicle) demands a considerably increased level of maturity and dependability. State of the art ECAS vehicle centralized architectures and classic safety and security mechanisms cannot support resilience in modern autonomous systems characterized by extensive interconnected embedded applications without being connected to the edge and cloud infrastructure. Therefore, autonomous systems need to address safety- and security-related flaws and provide resilience guarantees in highly heterogeneous and dynamic environments.

The relationships between ML, security and privacy for ECAS vehicles is illustrated in Figure 9.

Nascimento et al. (2020) provide a systematic literature review to offer an overview of AI's impact on autonomous vehicle safety. The paper argues that the literature reviewed proposes conceptual models and frameworks that bring essential contributions, but they are mostly not tested in actual set-ups. The AI techniques achieved good results, but they are not fully repeatable and reproducible, and the error rates do not confirm that they are suitable for real deployments in autonomous vehicles considering missing consistent hazard analysis. Virtual and physical validation of AI-based systems to the high-dependable and trustworthiness levels is required for fully autonomous SAE level 5. Safety certification of non-deterministic control systems is still a challenge.

Safety is firmly interrelated to end-to-end security for ECAS vehicles when considering communication via public or private networks. For specific missions, the autonomous vehicle can be remotely controlled, enabling the external "driver" to act on the 


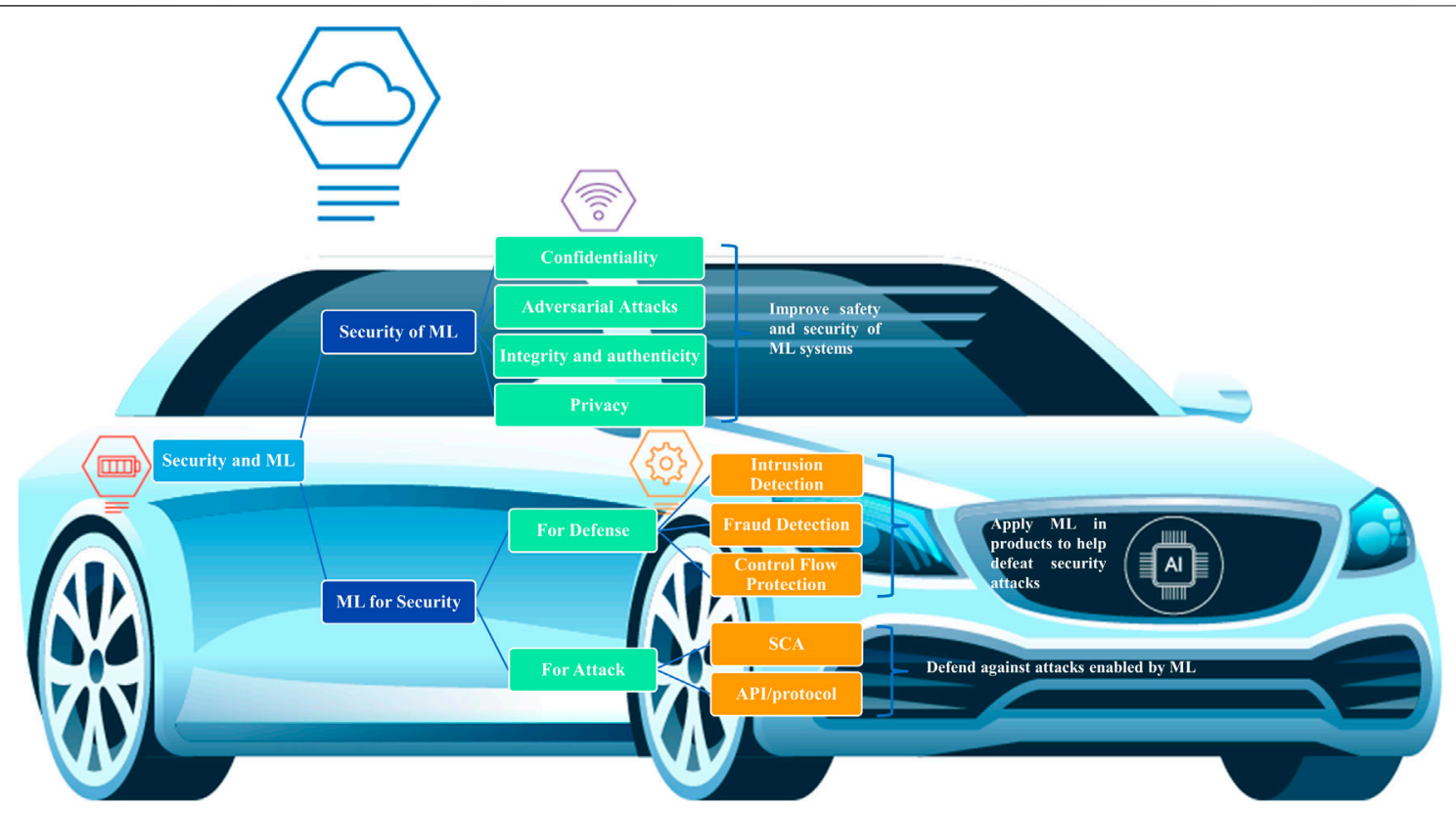

FIGURE 9 | ML, security, and privacy intersection for ECAS vehicles.

steering and braking system. Security is critical to protect the ECAS vehicles from any unauthorized persons, machines, and $\mathrm{HW} / \mathrm{SW}$ digital agents to control the functions related to the vehicle domains that can drive the vehicle to damage other vehicles, properties, humans, animals, etc. End-to-end security is paramount for the ECAS vehicles mobility applications, and access to the safety- and mission-critical components must be restricted and continuously monitored to avoid cyber-attacks and misuse. Munasinghe and Murtaza (2020) present several security objectives to minimize the potential vulnerabilities, which need to be considered in the security system's design. Confidentiality requires that the sensitive information is revealed and published only to authorized individuals or systems. Integrity correlates to the accuracy and consistency of the sensitive data/information transmitted through the network that needs to be protected and adequately maintained to avoid that data is compromised by unauthorized parties and altered in an unauthorized way. The availability requires that the network resources (equipment/bandwidth/data/information) are accessible and available to any authorized person or system as they are required. The authenticity of the transmitters/ receivers of messages communicated through the network should be verified so the parties are authenticated to whom they claim they are. The authorization must be implemented to provide the persons and systems the specific access rights and privileges to access sensitive information and functions. Nonrepudiation refers to the assurance that nobody can deny the truthfulness or validity of the data/information dispatched through the network.

In the ECAS vehicles, there are several access points for vehicle information, and various comprehensive information access strategies are developed by the automotive industry. Due to high-economic and liability risks in the future ECAS vehicles development, the OEMs will control the most extensive data sets, and they will provide means to make the information accessible and shared through their back-end systems. An overview of information security and privacy issues for ECAS vehicles is presented in Figure 10.

The V2X interfaces to access vehicle safety and missioncritical information and messages exchanged with other vehicles, and the OEMs keep the infrastructure with no information access for third parties.

The ECAS vehicle OBD-II port is employed for transmitting information via dongles, and the port is accessible for third-party solutions. The port can be restricted due to security risks and the proliferation of secured OTA solutions.

In this paper, the concept of trustworthiness is considered critical for ECAS vehicle technologies and applications. The concept builds on the system engineering practices and the fulfilment of dependability characteristics, including system properties such as availability, connectability, maintainability, privacy, reliability, resilience, security, and safety. These properties definitions follow the product quality model proposed by ISO/IEC 25010 (2011) standard.

Further research is needed in Fault Injection Testing for AI, Failure Modes and Effects Analysis (FMEA) for AI, AI safeguards, AI safety envelopes, and AI redundancy for ECAS vehicles. Developing explainable AI techniques, AI fault forecasting, and cognitive V2X communication support the development of fail-operational technologies providing solutions for hardware and software redundancy.

\section{Residual Risk}

A key element for the safety of ECAS is the implementation of fail-operational techniques, redundancy, and reduction of the residual risk. 


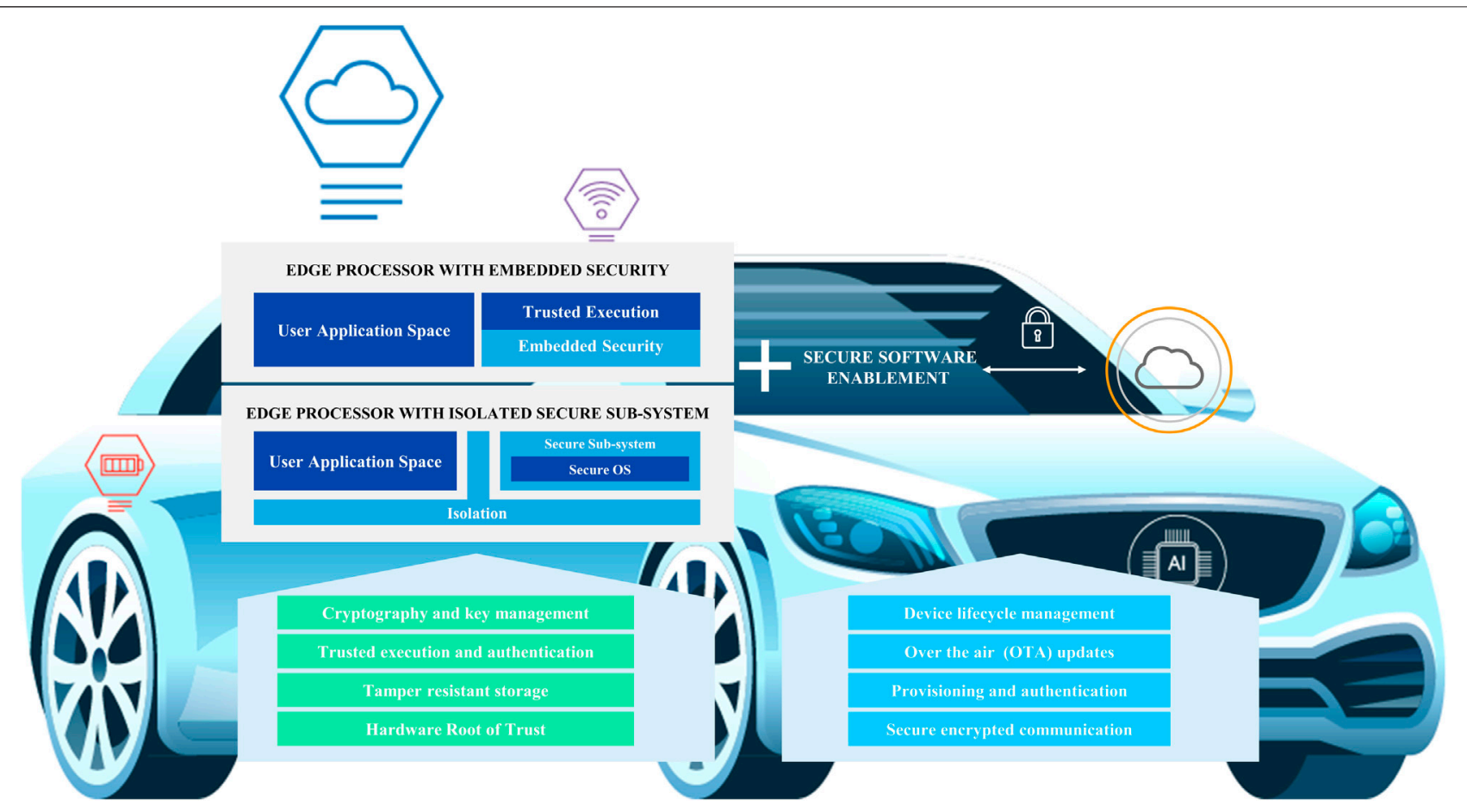

FIGURE 10 | Information security and privacy issues for ECAS vehicles.

A risk is considered an uncertain event or status, and the risks can be classified as identified and unidentified risks. Identified risks include residual and secondary risks that require a response operation to control them if required. Residual risks are the risks that are expected to continue after the planned responses of risk have been taken, as well as those that have been deliberately accepted. ISO 27001 (2013) defines residual risk as "the risk remaining after risk treatment." On the other hand, secondary risks are the risks that appear as a direct consequence of implementing a risk response. It is critical for ECAS vehicles that the limits of the risk threshold are defined to determine if the amount of residual risk is acceptable or not. If the residual risk is not accepted, additional risk operations should be taken to minimize its effects. The limits of the tolerable risks are presented in Figure 11.

The risk identification process for ECAS vehicles must analyze and reduce residual risks and avoid safety issues. A response operation or a contingency action must be created. If they do not require a response operation or a contingency action, the risks need to be monitored. Contingency actions can be used to repair the risk's potential damages.

A model-based approach to safety to enhance the existing functional safety and ASIL (ISO 26262, 2018) requirements in the automotive industry must cope with multi-agent environments. The model called Responsibility-Sensitive Safety (RSS) (ShalevShwartz et al., 2017; Chai et al., 2019; MobilEye, 2019) implements an interpretation of the duty of care from Tort law, which affirms that a person should exercise "reasonable care" while performing acts that could harm others. RSS applies to autonomous vehicles as a rigorous mathematical model formalizing an interpretation of the law. The concept focuses on the following elements: interpretation of the law that should be sound in the sense that it complies with how people interpret the law, the interpretation should lead to an applicable driving policy, meaning it will lead to an agile driving policy rather than an overly-defensive driving, the interpretation should be efficiently verifiable in the sense that we can rigorously prove that the autonomous vehicles implement the interpretation of the law correctly. The RSS aims to be agnostic of the implementation and is proposed to be accepted as a global safety model. The concept introduces a "semantic" language consisting of units, measurements, action space, and specification as to how they are incorporated into sensing, planning, and actuation of the autonomous vehicles comparable the existing driving policy. The RSS adheres to the following five safety rules:

- Do not hit the vehicle in front (longitudinal distance)

- Do not cut in recklessly (lateral distance)

- Right of way is given, not taken

- Be cautious in areas with limited visibility

- If the vehicle can avoid a collision without causing another one, it must

RSS formalizes human understandings of safe driving, using mathematical formulas and logical rules that are transparent and verifiable. These rules establish the common-sense behavioral characteristics that humans would characterize as leading to safe driving. The aim is that the autonomous vehicle should drive prudently enough so that it will not be the cause of an accident and carefully enough so that it can compensate for the mistakes of 


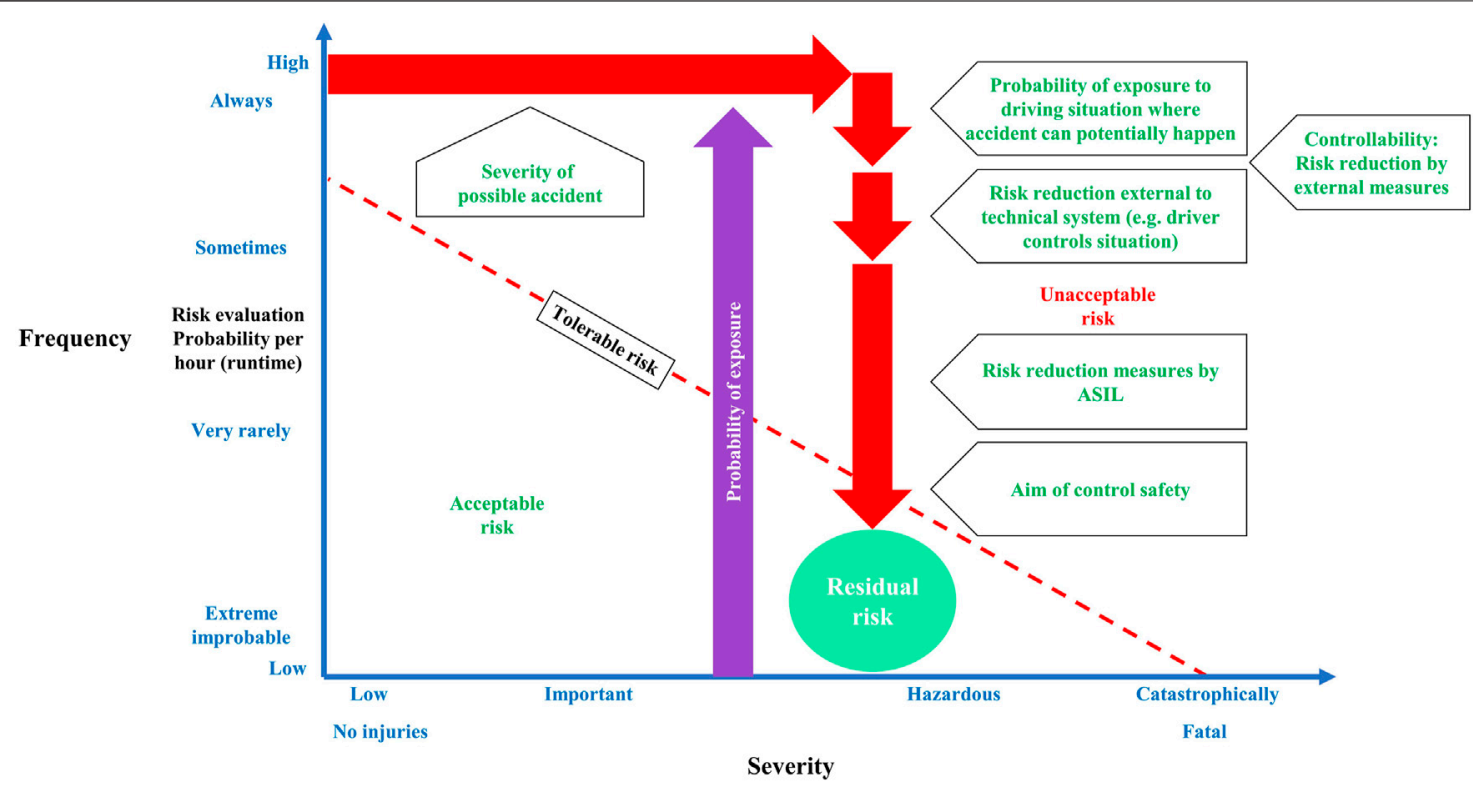

FIGURE 11 | |llustration of limits of the tolerable and residual risks.

others. The IEEE P2846 (IEEE P2846, 2020) defines the minimum set of reasonable presumptions used in foreseeable situations to be analyzed for road vehicles in developing safetyrelated models that are part of autonomous driving systems and considers the RSS model as the initial point for safety considerations in autonomous vehicle decision-making.

ISO has adopted the Safety First for Automated Driving (SaFAD) paper (Wood et al., 2019) as a technical report, which is considered the first step toward standardization and included the RSS in the Drive Planning Element.

\section{INTRINSIC AND EXTRINSIC INTELLIGENCE FOR SUSTAINABLE GREEN MOBILITY}

ECAS vehicle's benefits include the reduction/elimination of accidents, providing crash-free solutions, and improving vehicle safety by continuously monitoring the environment while using the upgraded road infrastructure and managing traffic flow with reduced maintenance costs. The ECAS vehicles architecture provide the partitioning of software and hardware as illustrated in Figure 12.

The deployment of ECAS vehicles using AI technologies for the vehicle's design and the mobility services and applications optimizes the vehicle functions, operations, and missions. The AI role in ECAS vehicles domains is illustrated in Figure 13.

Through V2X connectivity, travel times can be estimated via real-time, predictive assessments on all routes, and the reduction in driving tasks enables passengers to use travel time more efficiently.

To implement the intrinsic and extrinsic intelligence at the vehicle level and mobility mission level for optimizing the different autonomous functions, seamless, anytime, anywhere intelligent connectivity is essential. Intelligent connectivity (e.g., wired/ wireless, cellular 5G/6G, network slicing, satellite) based on different technologies (He et al., 2018) that coexist and provide the fail-operational requirements may impose that the $\mathrm{V} 2 \mathrm{X}$ modules are running based on several communication technologies. If one $\mathrm{V} 2 \mathrm{X}$ module experienced a hardware failure, connectivity, or GPS loss, a logic mechanism could trigger a redundant parallel system based on another technology to begin responding to outputs from the other V2X unit.

The extrinsic intelligence of ECAS vehicles is linked to swarm intelligence (SI) based on the collective behavior of elements in decentralized and self-organized mobility fleets. As IoV-based fleets are heterogeneous and consist of intelligent vehicles with multiple perception sensors and use decentralized algorithms to solve complex environment recognition and navigation problems using different AI techniques in mobility applications implementations. The AI algorithms can solve the challenging issues of optimizing the behaviors of fleets of ECAS vehicles addressing the dynamic properties, vehicle mobility, sensor fusion, wireless communication, and information provision. Swarm intelligence solutions are applied in fleet optimization problems where real-time actions are managed and supervised efficiently. Swarm intelligence algorithms like ant colony optimization can play essential roles in normalizing the fleets of ECAS vehicles mobility processes.

The extrinsic intelligence, including smarm computing capabilities, enhance cooperative interaction between individual ECAS vehicles, each with their intrinsic intelligence and autonomy, and working collectively in harmony for the benefit of the collective traffic and interacting in real-time with the other vehicles, traffic participants (e.g., passengers, drivers, pedestrians, and infrastructure such as traffic lights, lighting poles, buildings, IoT, edge computing, cloud infrastructure, etc). 


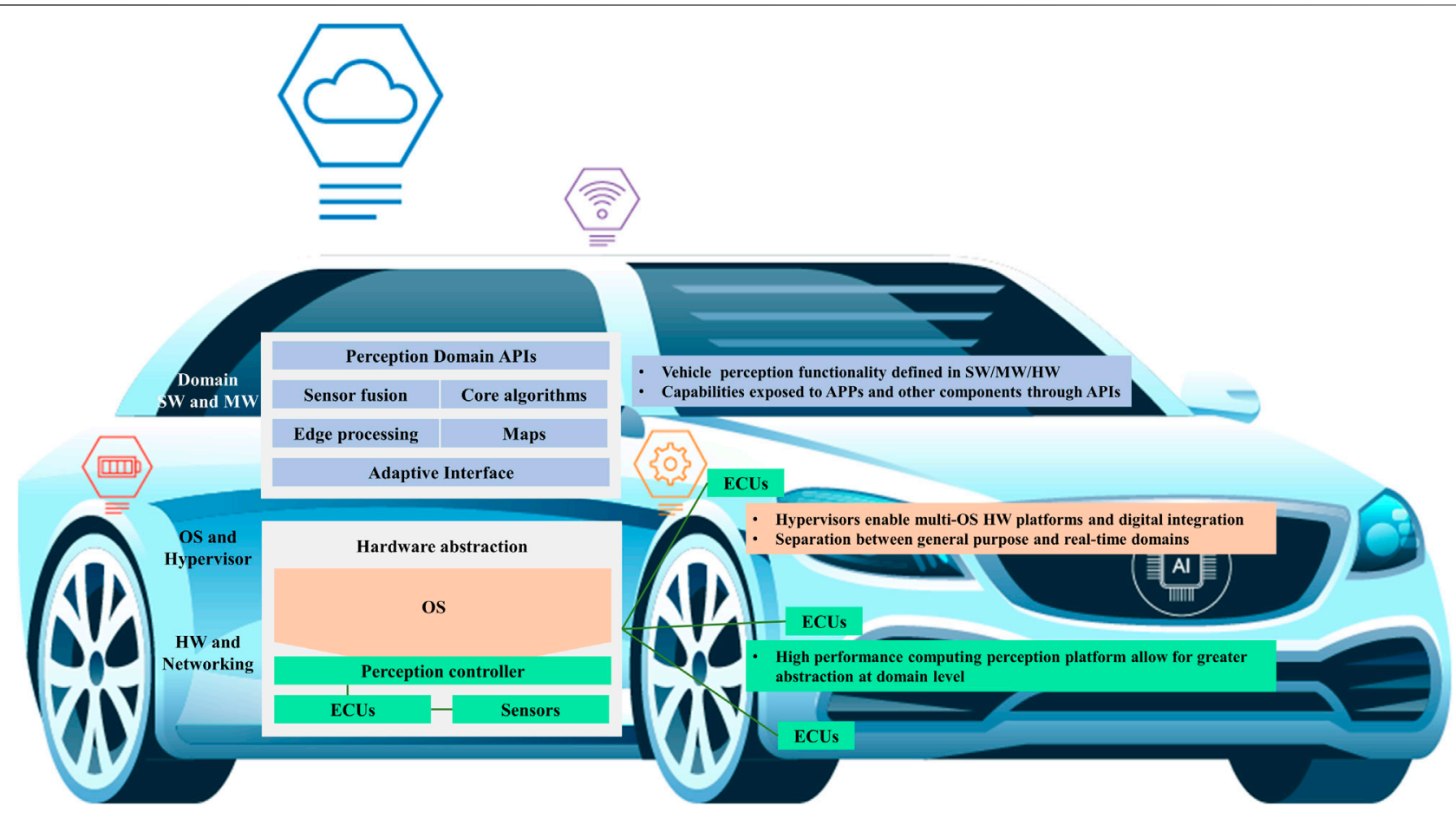

FIGURE 12 | Hardware and software partitioning in ECAS vehicles.

The concept of smarm prediction can be used in fleets of ECAS vehicles to forecast traffic routes and optimization. Traffic and vehicle interactions simulations can determine the optimal vehicles' behavior based on constraints like energy consumption, shortest route, time-efficient routes, etc. can be integrated into the simulation models, including digital twins of the ECAS vehicles and virtual maps and infrastructure that mimic the real-time scenarios.

The computing requirements for implementing intrinsic and extrinsic intelligence have advanced the development in edge computing that evolved in developing technologies that fulfill the specific area's needs and granularity such as meta edge, deep edge, and micro-edge as presented in Figure 14. The micro-edge and deep edge technologies apply mainly to the intrinsic on ECAS vehicle intelligence, while meta edge applies both to intrinsic and extrinsic vehicle intelligence.

The edge computing layers are combined with AI, SI algorithms on devices inside and outside the ECAS vehicles allowing the information to be processed in real-time without connecting to a cloud.

Energy, communication, and extremely information-intensive AI processes required in overall traffic coordination, vehicle maintenance, and interactions among fleets are performed in clouds as they mandate a large amount of computing power and energy.

The evolution of edge computing and swarm intelligence allows the ECAS vehicles to operate autonomously and interact locally with other vehicles and infrastructure in all real-time operations. The intrinsic and extrinsic intelligence enables $\mathrm{ML}$ and $\mathrm{DL}$ algorithms that run on the vehicle perception sensors, in the vehicle devices, on the vehicle central computing unit, and in the meta-edge infrastructure.

Providing performant mobility solutions by deploying ECAS vehicles is challenging, requiring multi-parameter optimization for carbon reduction, energy efficiency, shortest route, maximum speed, minimum congestion, and landscape configuration. AI, SI, IoT, and edge computing are the technologies that can sustain or even enhance future mobility solutions under these constraints.

ECAS vehicles rely on ML to resolve challenging tasks in perception and motion planning. The hardware, software, and embedded safety standards have not sufficiently evolved to address AI and ML safety challenges like interpretability, verification, and performance limitations. The lack of human interpretable specifications implementation, run-time monitoring functions, transparency, testing, verification, and virtual validation are significant challenges for ML deployment and the homologation of ECAS vehicles.

The use of ML for increased intrinsic and extrinsic intelligence and enhanced safety requires an inherent safety design that starts with the design specifications, implementation strategy, virtual validation for providing fail-operational properties, and minimizing the residual risk by increasing the safety margin.

Edge AI and SI are becoming increasingly relevant for ECAS vehicles as they are operating autonomously in local environments and need to exchange information efficiently in real-time. The edge $\mathrm{AI}$ and SI are even more critical considering the perception domain of the vehicle, including the advanced perception sensors and actuators and the computer vision capabilities, and the 


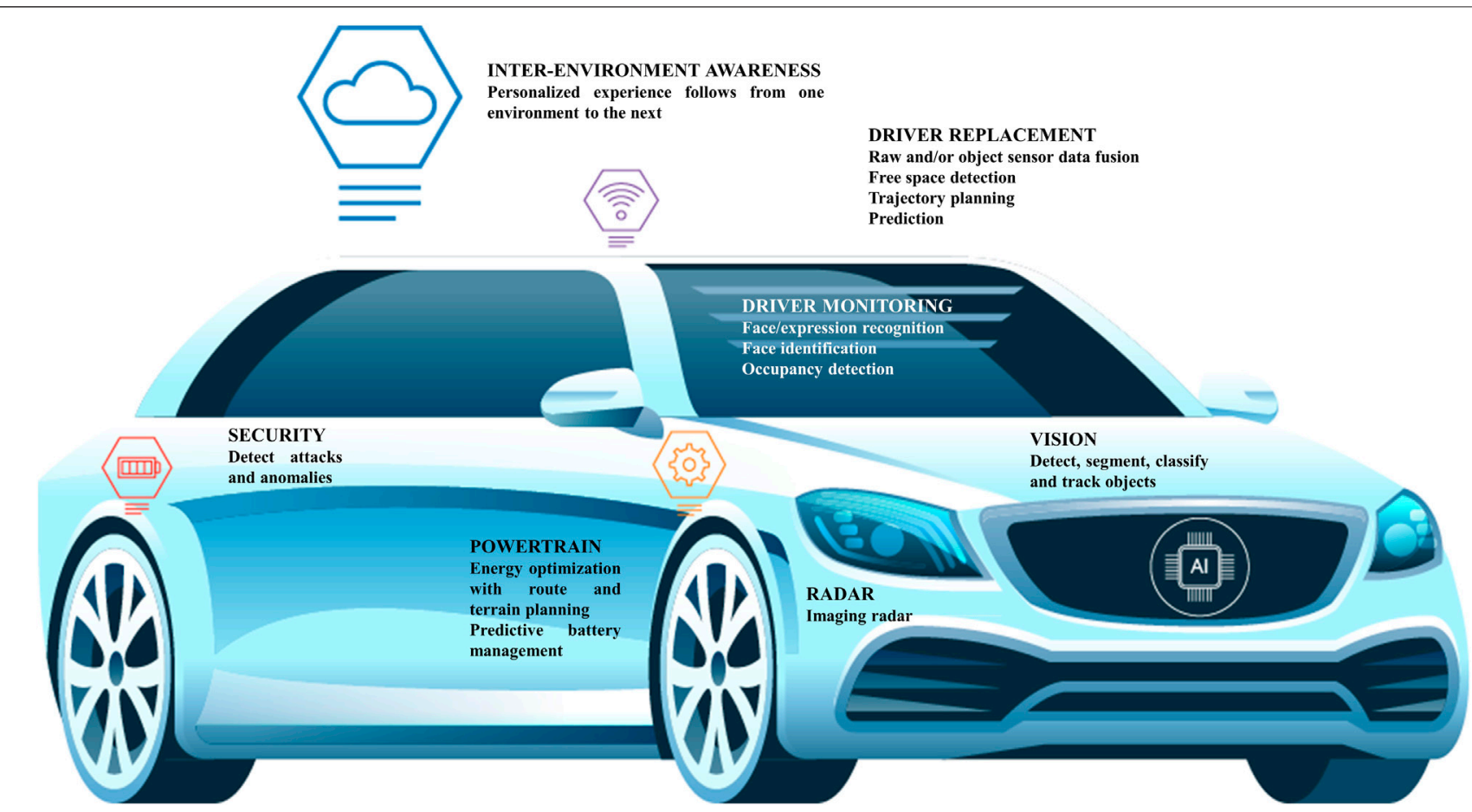

FIGURE 13 | Al role in ECAS vehicle.

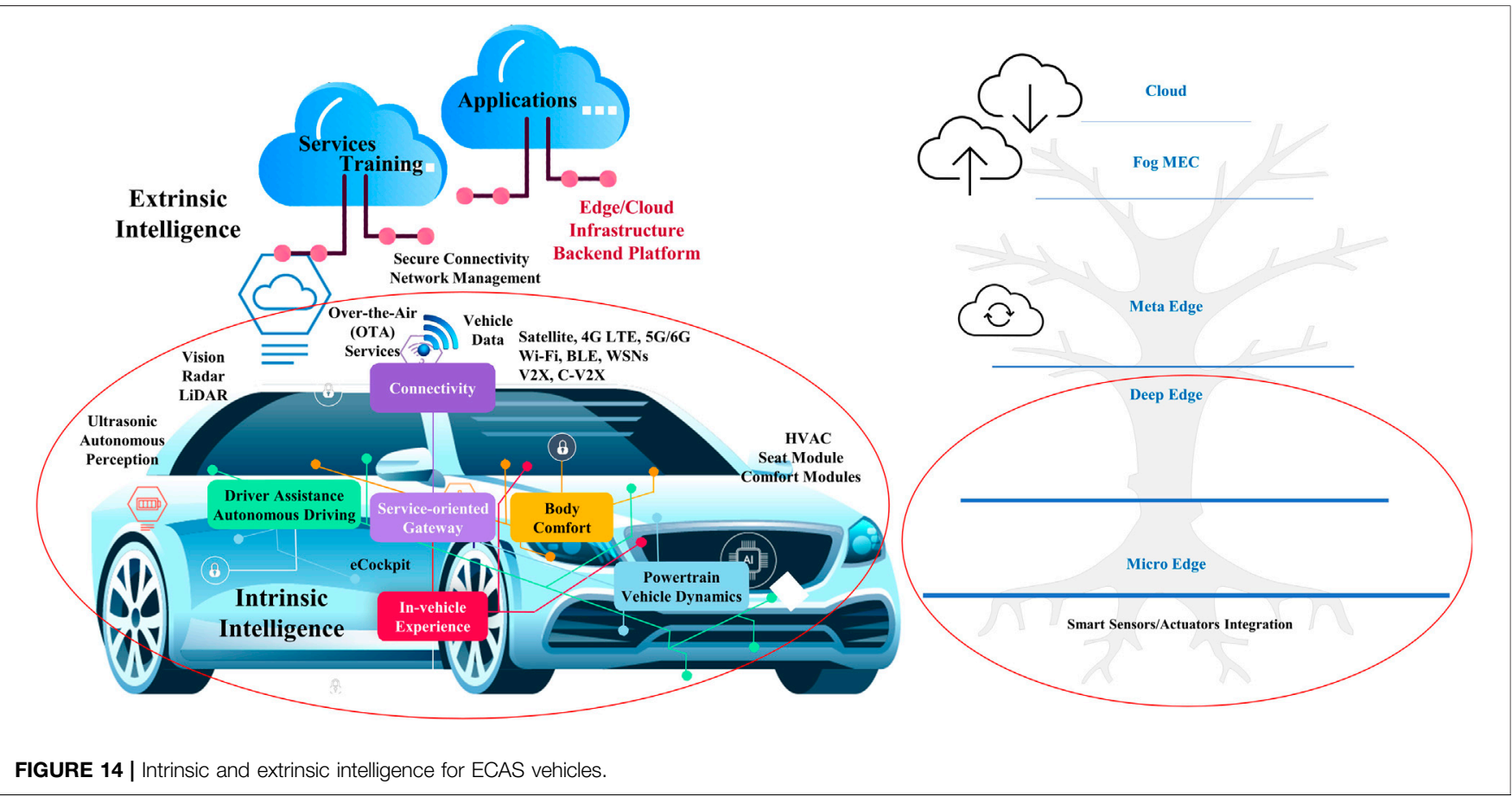

algorithms that are essential for implementing the intrinsic and extrinsic intelligence capabilities.

The time delays in the transmission of data from these sensors could have catastrophic safety consequences. ECAS cannot experience low latency transmission while detecting objects on the street and fast response time is so critical that the perception sensors/actuators themselves must have micro-edge AI capabilities that allow them to analyze and classify images locally. 
TABLE 3 | ECAS vehicle future research challenges.

Future research challenges in automotive intelligence

\section{ECAS vehicle domain - Energy}

Research on new batteries and new battery management systems based on Al algorithms to enhance the charging/discharging efficiency and increase the battery lifetime by using charging patterns favorable to battery health is needed.

Further work must include intelligent techniques and standard interfaces for preparing the batteries for the second life and even for recycling by monitoring the batteries' entire life cycle. New research is needed on wireless communication technologies for the battery systems combined with Al methods to optimize the battery pack's communication and reduce the wiring.

Innovation is needed to develop efficient wireless charging technologies to reduce the energy loss for ECAS vehicles and autonomous energy equipment to electric vehicles enabled by the dynamic on-road and off-road wireless (e.g., dynamic charging roadways) power transfer.

New high-performance, safe, and cost-effective, dynamic electric vehicle charging solutions combining robotic arms chargers, wireless charging pads are further needed to develop and improve the energy system for ECAS vehicles. These advancements are required to find new solutions for autonomous vehicles to charge themselves, optimize the use and type of energy utilized (source of energy and the renewables content in the energy mix, etc.), and build a seamless decentralized network of charging units.

\section{ECAS vehicle domain - Powertrain and vehicle dynamics}

The powertrain and vehicle dynamics developments with integrated and distributed powertrains both for fuel cell and the pure electric powertrains are needed, including extreme engine downsizing for multiple energy harvesting/regeneration and structural supercapacitors and batteries. The developments demand new forms of rotating electrical machines, energy storage, energy harvesting, and regeneration combined with energy-efficient power electronics.

New research is needed for vehicle dynamics and powertrain optimization using real-time data from the missions and applying Al algorithms for co-optimizing the vehicle functions.

Research on energy efficiency and energy savings for the powertrain operation based on the different mobility scenarios (e.g., platoon traffic integration of information from the environment, infrastructure, traffic signals, traffic lights, etc.).

Research is needed in green and efficient mobility and developing a co-optimization framework to address the optimal energy consumption and vehicle dynamics based on the trajectory prediction, driving profile (e.g., acceleration, braking, stops, etc.), and environmental conditions.

Further work is needed for virtual verification and validation and simulation tools to optimize real-time the power train and vehicle dynamics.

\section{ECAS vehicle domain - Connectivity}

Further work is needed to provide scalable solutions and techniques and Al algorithms for optimizing the use of wireless/cellular networking, edge-to-cloud protocols, and middleware-to-cloud provisioning systems.

The multi-vehicle OTA updates and upgrades must be investigated to offer new concepts for end-to-end security, security protocols, edge hardware and storage, personal area network (PAN), protocols, middleware systems, sensor costs and resources, and cloud provisioning layers.

Embedded systems in ECAS vehicles require connected technologies that rely on cryptography and Al-based technologies to secure data and authenticate communications. The cryptography used needs to withstand attacks from future quantum computing technologies. Research should focus on post-quantum cryptography, including new cryptographic algorithms (e.g., public-key algorithms) efficiently secured against attacks using quantum computers.

Due to the technological challenges and the need for redundancy in fail-operational autonomous vehicles, global hybrid V2X solutions (e.g., wireless, cellular, satellite) should be further investigated to provide flexible technology deployments and upgrades (e.g., timing, model, market).

Adaptive compensation could be another future research area to allow the V2X system to compensate for a failure in one or more components by relying more on other complementary components or processes that need to be made available. For the case of a GPS receiver that suffers a hardware failure and generates noisy or intermittent data, the state estimation system should reduce the weight of the GPS data and increase the weight on other available sources of information (e.g., in-vehicle navigation system) to continue to provide a robust, filtered solution. The strategy can be applied for V2X systems that fuse data from multiple sources for localization.

The solutions should address the compensation techniques applied for a limited amount of time (e.g., state estimator drift could cause the vehicle to lose track of its absolute position over time if GPS or other absolute data are not acquired from the satellites). The strategy can be applied to the use of different satellite systems (e.g., GPS,

GALILEO, etc.).

Further research is needed to identify different fail-operational mechanisms relates to degraded modes of operation that could allow the V2X system to continue functioning after a failure. Operating the vehicle at a reduced speed in a defined environment is a valuable tool for mitigating faults or failures associated with constrained resources (e.g., GPS loss, network bandwidth, processing power, processing latency/lag). The strategy provides the V2X system with the decision window to evaluate a scenario and make navigation changes.

New solutions and research are needed to optimize the wireless/cellular connectivity and edge computing (Vermesan et al., 2020a) processing for ECAS vehicles while introducing techniques to reduce interferences.

\section{ECAS vehicle domain - Perception}

The automotive intelligence developments need to extend the performance, cost reduction, energy-efficiency computing, and cognition of the perception systems for safely operating in all roads, weather, and traffic conditions. The research should include developing new Al-based methods for identifying roads with highly deteriorated or no lane marking, no defined lanes, having potholes, or including mountainous conditions and tunnels where external signals for direction are not clear.

Techniques for the ECAS vehicle to identify (directly or via the infrastructure) different weather/road conditions that may occur and algorithms to interpret the effects on the driving capabilities of the vehicles are further required.

Research on dealing with unexpected conditions and unpredictable traffic participant's behaviors in dense or regular traffic and the link to cognitive decision-making and optimization of the vehicle domain functions are required.

Open research issues remain on managing and optimizing the vehicle's internal systems aligned with external conditions, traffic situations, infrastructure capabilities, and traffic participant's behavior. To provide energy-efficient, sustainable green solutions, the vehicle level's efficiency and traffic level need to be addressed together.

New solutions and research are needed to optimize the wireless/cellular connectivity and edge computing (Vermesan et al., 2020a) processing for ECAS vehicles while introducing techniques to reduce interferences. Radar combined with low-cost solid-state 3D LiDARs and advanced cameras, odometry sensors, and low-cost GPSs, requires more research and development. 
TABLE 3 | (Continued) ECAS vehicle future research challenges.

Future research challenges in automotive intelligence

ECAS vehicles use LiDARs and radars for navigation, with the radar detecting reflections of radio waves from surrounding objects by continuously emitting radiofrequency waves reflected from the surrounding vehicles and other objects near the road. The reflection time measured is used to calculate the distance between the vehicle and the objects, and proper actions are then taken based on the radar readings.

Considering that tens to hundreds of vehicles use the radars on the road, the vehicle must accurately distinguish between the own (reflected) signals and the signals (reflected or transmitted) from other vehicles. More effort must be dedicated to techniques and solutions to use multiple radio frequencies and other intelligent radar implementations to reduce inferences.

Al technologies' significance is increasing due to the unpredictable environment and behavior of the surrounding objects and traffic participants with whom the ECAS vehicles interact during the missions. The further development of computer vision-related algorithms and mechanisms like object detection, perception, scene identification, reconstruction, and estimation require advanced $\mathrm{ML}$ and $\mathrm{DL}$ mechanisms.

More research is needed in Al to interpret and categorize the driving environment by the ECAS vehicles. The driving environments should include tests covering weather conditions such as driving in fog, adverse weather events, ice, and snow. The perception system LiDARs' and camera's limited exposure must be further enhanced using multimodal sensor fusion and point edge computing analysis for object classification. More reliable object detection techniques, mathematical models, and simulations are needed to imitate reality and provide the best parameters and configurations to adapt to changes in surroundings using DL and CNNs, to achieve a high level of arbitrary accuracy to solve autonomous vehicle's perception requirements.

Extended work is required to develop prediction algorithms and techniques essential for the autonomous vehicle's computer vision to make decisions in an occlusive (e.g., noise in sensory data, unpredictable behavior, limitations of sensors, etc.) and uncertain environment that affects the information's reliability.

Radar combined with low-cost solid-state 3D LiDARs and advanced cameras, odometry sensors, and low-cost GPSs, requires more research and development.

\section{ECAS vehicle domain - Autonomous driver and cognition}

The use of Al techniques in the development of the ECAS vehicle domains increases the integration of these methods into SW/HW testing and virtual validation, including Albased digital twin's development for real-time virtual environment simulation. Further investigation of Deep Neural Network (DNN)- based software where the software learns and adapts is an important research area to be pursued.

Other open issues in the ECAS vehicles technologies relate to the cognition and decision-making processes and techniques used in uncertain environments. The vehicles have to distinguish between the own local environment/situation and the holistic approach of addressing the traffic situation considering the interaction with all the other traffic participants.

The cognition and decision-making processes for ECAS vehicles are a multi-dimensional issue that depends on various elements (e.g., autonomous vehicle's behavior, neighbors, perception, prediction, sensor fusion/data processing, components' calibration, etc.). Further development of the decision-making mechanisms in autonomous vehicles based on Al. ML, DL, multi-policy decision making, Markov decision processes are needed to make the processes reliable and resilient.

Cognition and decision-making need to be addressed holistically by including the intrinsic and extrinsic optimization and increase the efficiency of V2X communication in cooperative crowdsensing, crowd-perception, and other vehicles' behavior. The cooperative behavior of ECAS vehicles impacts optimizing the traffic and the use of resources at the fleet level.

The research on the use of edge computing and loT extend to the development of high-definition (HD) dynamic maps and the integration with the maps generated by GPS systems to provide more accurate road details (e.g., lane dimensions, distance from pedestrians/objects/vehicles, curb height, etc.).

The real-time processing and analysis of the information received by the ECAS vehicle remain a challenge, and efforts are needed to reduce the complexity of the software while increasing reliability. These requirements are extended to validation and testing, trust, software quality, computational resources, safety and reliability, privacy, and security ECAS vehicles are mission-critical and safety-critical systems that require fine-tuned complete validation and testing to ensure that all the stringent requirements are met. Software quality must balance the degree of complexity that goes into autonomous vehicles due to the cognition functions that mimic the human brain and/or behavior and the need for efficient, cost-effective implementation, validation, and testing (Koopman and Wagner, 2016).

\section{ECAS vehicle domain - Body and comfort}

Research is needed on exploring the relationship between comfort and the ECAS vehicle longitudinal and lateral movement, along with its positioning in the road, vertical loadings, type of maneuver, weather conditions, environment type, presence of other traffic participantsetc.

Research is needed to create a framework for virtual simulation of autonomous driving scenarios that can evaluate a broader population of potential AV users close to real-world settings within various driving conditions.

The management of ride comfort and the correlation between the driving scenarios, passenger's tasks during the ride, and the vehicle dynamics need further research Further research needs to establish the broader factors of comfort, how these systems influence one another, and how different aspects might adapt over time and scenario.

\section{ECAS vehicle domain - Vehicle experience}

The technical challenges in the vehicle experience are related to multimodal interface and immersive experience and the evolution of new virtual and augmented reality tools for ECAS vehicles.

As the passenger compartment of the ECAS vehicle could be remodeled into a cinema, living area, games room, or even a co-working space, new research is needed for defining the interfaces between passengers and vehicles.

Innovative solutions of the in-vehicle infotainment system user interface (UI) that forms the hub of a vehicle's electronic system is another area that requires more research and innovation to address the needs in multimedia content and the immersive visual experience.

Further work is needed to integrate the in-vehicle infotainment system and build integrated communication services to create a symbiotic relationship between vehicles, passengers, smartphones, tablets, and computers.

The use of all edge AI and SI layers efficiently increases the vehicle's sensor fusion capabilities, improves the vehicle's local decision-making, reduces unnecessary data transmission, minimizes communication interruptions, and improves vehicle's cooperative capabilities.
ECAS vehicles must continually be scanning the surrounding environment and evaluating the situation, making corrections to its trajectory based on contextual conditions and local events. Real-time data processing is critical for ECAS vehicle mission-critical 
applications, and the onboard edge AI and SI systems are responsible for data storage, sensor fusion, cognition, and analysis. The edge AI and SI systems enhance the autonomous capabilities of ECAS vehicles and optimize the intrinsic and extrinsic intelligence functions. The optimization of the driving functions of the ECAS vehicles at the vehicle level combined with the optimization of the mobility applications improve energy efficiency and provide efficient driving, fuel-efficient journeys, and efficient infrastructure.

ECAS vehicle's developments generate new models for ECAS vehicle ownership and extend opportunities for vehicle-sharing, ride-sharing by bringing on the market new business models and scenarios based on the convergence of technologies and the integration of solutions across industries (e.g., automotive, energy, ICT, buildings, transportation).

The optimization of intrinsic and extrinsic intelligence for sustainable green mobility applications and services requires that the vehicles are not treated solely as individual autonomous machines but as participants in missions, applications and services. The optimization at intrinsic and extrinsic level empowers the efficient use of materials, resources, computing power, energy, and related emissions. The ECAS vehicles are integrated into fleets and IoV applications and optimize the intrinsic and extrinsic operations and functions by exchanging and sharing historical and real-time information using the connectivity channels and the infrastructure edge and cloud platforms.

Mobility stakeholders are using federated platforms and are creating information lakes, spaces, marketplaces where data is available through regulatory decisions for use in mobility different scenarios and missions. Information sharing among ECAS vehicles, and their stakeholders is critical for intrinsic and extrinsic intelligence optimization with information flowing continuously in real-time among the vehicles and infrastructure. The collection, processing, exchange, and sharing of information must consider the data's privacy and ownership by anonymizing and encrypting the data from sensors/actuators, sensitive vehicles, and participant's data in mobility applications/services.

\section{FUTURE RESEARCH CHALLENGES IN AUTOMOTIVE INTELLIGENCE}

This section discusses various current open research and innovation topics and gives recommendations for future research guidelines and directions on different issues related to automotive intelligence and ECAS vehicle technologies and applications.

To design ECAS vehicle systems capable of controlling a vehicle's performance similar or better than a person in all conceivable conditions is a challenging multidisciplinary endeavor. The ECAS vehicles have a complex E/E architecture that integrates different functional domains, including sensors/ actuators, electronic control units, advanced AI techniques/ algorithms, processing, perception, propulsion, energy, and connectivity capabilities to execute the mobility functions assigned to different missions.

Extensive research and innovation are needed on the $\mathrm{E} / \mathrm{E}$ architectures and the optimization of domains and virtualization of functions and containerization on the vehicle's processing platforms and the move toward federated vehicle/edge/cloudcentric based on distributed intelligence at the vehicle and infrastructure level.

The complexity of the ECAS systems requires a new approach to developing vehicle operating systems and various data fusion systems. Challenges and open issues are related to the orchestration of resources when using virtualization/ lightweight virtualization, virtual machines, microservices, containers, and unikernels for running mobility applications, services on the ECAS vehicle platform.

The future research challenges in automotive intelligence across the ECAS vehicle domains are summarized in Table 3.

\section{SUMMARY AND CONCLUSIONS}

Automotive industry is transforming and evolving from a classical value chain to a flexible and adaptable value network. The transformation is driven by the technological development of the ECAS vehicles architectures, systems intelligence, and the integration of new computing paradigms at the edge with embedded cognitive and swarm capabilities into the vehicle domains and data infrastructure to provide holistic intrinsic and extrinsic intelligence into green mobility applications, while at the same time ensuring robustness, safety, security, and privacy.

The automotive intelligence is embedded into perception sensors, propulsion engines, actuators, electronics, HW/SW components, connectivity systems, applications, and services. The technology development, innovation in the field, AI-based methods, and virtual validation and testing allow ECAS architectures to advance vehicle's capabilities.

In this article, we refined and expanded on the latest concepts related to ECAS vehicles, highlighting the extensive automotive intelligence opportunities to extend the vehicle's capabilities and change the business models and concepts for future mobility and transportation.

The article gives an overview of the automotive intelligence for ECAS vehicles, the developments, new architectures, AI techniques and methods used for the integration of autonomous functions in next-generation vehicles. These advancements imply the convergence of technologies such as IoT, edge computing, swarm systems, robotics, intelligent connectivity and trustworthiness frameworks for the deployment of sustainable green mobility solutions combining intrinsic and extrinsic intelligence for optimizing the cooperative and collaborative vehicle functions.

Finally, an overview of standards AI and ECAS vehicles are subject to and a description of standardization activities being undertaken by different SDOs is provided.

\section{AUTHOR CONTRIBUTIONS}

OV coordinated the structure and elaboration of the paper and the alignment and integration of individual contributions. All authors contributed ideas, content, perspectives, and references 
based on the work in different common projects and discussed the manuscript.

\section{FUNDING}

This work was supported by the European Commission within the European Union's Horizon 2020 research and

\section{REFERENCES}

ACEA (2020). European Automobile Manufacturers Association. ACEA Position Paper Artificial Intelligence in the Automobile Industry. Available at: https:// www.acea.be/uploads/publications/ACEA_Position_Paper-Artificial_Intelligence_ in_the_automotive_industry.pdf.

AEC Q100 (2014). Failure Mechanism Based Stress Test Qualification for Integrated Circuits. Available at: http://www.aecouncil.com/Documents/ AEC_Q100_Rev_H_Base_Document.pdf Ahmad.

AECC (2021). Automotive Edge Computing Consortium. Available at: https://aecc.org/.

AI4DI (2019). Artificial Intelligence for Digitising Industry. Available at: https:// ai4di.eu/.

Alamoudi, Y. A., Ferrah, A., Panduranga, R., Althobaiti, A., Ferrah, A., Panduranga, R., Althobaiti, A., and Mulolani, F. 2019). "State-of-the Art Electrical Machines for Modern Electric Vehicles," 2019 Advances in Science and Engineering Technology International Conferences (ASET), Dubai, United Arab Emirates, 1-8. doi:10.1109/ICASET.2019.8714343

5G Americas (2020). Security Considerations for the 5G Era. White paper. Available at: https://www.5gamericas.org/wp-content/uploads/2020/07/SecurityConsiderations-for-the-5G-Era-2020-WP-Lossless.pdf.

Amparore, E., Beccuti, M., Collina, S., De Simone, F., Donatelli, S., Beccuti, M., Collina, S., De Simone, F., Donatelli, S., and Tango, F. (2014). "Cognitive Systems in Intelligent Vehicles - A New Frontier for Autonomous Driving," in 2014 11th International Conference on Informatics in Control, Vienna, Austria (Automation and Robotics (ICINCO)), 817-822. doi:10.5220/0005160808170822

AA (2021). Android Auto. Available at: https://www.android.com/auto/.

ANSI Ul 4600 (2020). Standards for Safety - Evaluation of Autonomous Products. Available at: https://webstore.ansi.org/Standards/UL/UL4600Ed2020.

ACP (2014). Apple CarPlay. Available at: https://www.apple.com/ios/carplay/.

ArchitectECA2030 (2020). Trustable Architectures with Acceptable Residual Risk for the Electric, Connected and Automated Cars - ECSEL Project. Available at: https://autoc3rt.automotive.oth-aw.de/.

Arena, F., and Pau, G. (2019). An Overview of Vehicular Communications. Future Internet 11 (2), 27-12. doi:10.3390/fi11020027

ASA (2020). Automotive SerDes Alliance. Available at: https://auto-serdes.org/.

Automotive Association 5GAA (2016). Automotive Association 5GAA. Available at: https://5gaa.org/.

AVCC (2019). Autonomous Vehicle Computing Consortium. Available at: https:// www.avcconsortium.org/.

AUTOPILOT (2017). AUTOPILOTproject Automated Driving Progressed by Internet of Things. Available at: https://autopilot-project.eu/.

AUTOSAR (2003). (AUTomotive Open System ARchitecture). Available at: https://www.autosar.org/.

Berger Roland Strategy Consultants (2014). Think Act Autonomous Driving. München: Roland Berger Strategy Consultants GmbH. Available at: https:// www.rolandberger.com/publications/publication_pdf/roland_berger_tab_ autonomous_driving.pdf.

Birnie, A. (2020). Using Vehicle-To-Cloud Connectivity to Extend Electric Vehicle Range. NXP White Paper. Available at: https://www.nxp.com/.doi:10.3997/ 2214-4609.202010892

Bockrath, S., Rosskopf, A., Koffel, S., Waldhör, S., Srivastava, K., and Lorentz, V. R. H. (2019). "State of Charge Estimation Using Recurrent Neural Networks with Long Short-Term Memory for Lithium-Ion Batteries," in IECON 2019 - 45th Annual Conference of the IEEE Industrial Electronics Society, Lisbon, Portugal, 2507-2511, doi.org/doi:10.1109/IECON.2019.8926815

Boldea, I., Tutelea, L. N., Parsa, L., and Dorrell, D. (2014). Automotive Electric Propulsion Systems with Reduced or No Permanent Magnets: An innovation programme funding, ECSEL Joint Undertaking project ArchitectECA2030 under Grant Agreement No. 877539, ECSEL Joint Undertaking project AI4DI under Grant Agreement No. 826060, ECSEL Joint Undertaking project SECREDAS under Grant Agreement No 783119 and ECSEL Joint Undertaking project AI4CSM under Grant Agreement No. 101007326.

Overview. IEEE Trans. Ind. Electron. 61 (10), 5696-5711. doi:10.1109/ TIE.2014.2301754

Bose, P. (2019). "Toward Self-Aware, Resilient Systems and Ethical Artificial Intelligence," O’Reilly Artificial Intelligence Conference, New York, April 15-18, 2019.

Bostrom, N. (2014). Superintelligence: Paths, Dangers, Strategies. Oxford: Oxford University Press. 978-0-19-967811-2.

Cao, Z., Hidalgo, G., Simon, T., Wei, S.-E., Hidalgo, G., Simon, T., et al. (2021). OpenPose: Realtime Multi-Person 2D Pose Estimation Using Part Affinity Fields. IEEE Trans. Pattern Anal. Mach. Intell. 43 (1), 172-186. doi:10.1109/TPAMI.2019.2929257

Car 2 Car Communication Consortium (2021). ITS-G5 and Sidelink LTE-V2x Cochannel Coexistence Mitigation Methods. White paper. Available at: https:// www.car-2-car.org/fileadmin/documents/General_Documents/C2CCC_WP_ 2091_Co-ChannelCoexistence_MitigationMethods_V1.0.pdf.

Casademont, J., Cordero, B., Camps-Mur, D., da Conceicao, L. A. M., Lalos, A., Vitale, C., et al. (2020). Multi-Radio V2X Communications Interoperability through a Multi-Access Edge Computing (MEC), “2020 22nd International Conference on Transparent Optical Networks, Bari, Italy. ICTON, 1-4. doi:10.1109/ICTON51198.2020.9203495

Chai, C., Zeng, X., Wu, X., Zeng, X., Wuand Wang, X. (2019). "Safety Evaluation of Responsibility-Sensitive Safety (RSS) on Autonomous Car-Following Maneuvers Based on Surrogate Safety Measurements," 2019 IEEE Intelligent Transportation Systems Conference (ITSC), Auckland, New Zealand, 175-180. doi:10.1109/ITSC.2019.8917421

de Geus, D., Meletis, P., Meletis, P., and Dubbelman, G. (2020). Fast Panoptic Segmentation Network. IEEE Robot. Autom. Lett. 5 (2), 1742-1749. doi:10.1109/LRA.2020.2969919

EN 17186 (2019). Identification of Vehicles and Infrastructures Compatibility Graphical Expression for Consumer Information on the EV Power Supply. Available at: https://standards.cen.eu/dyn/www/f?p=204:110:0 (FSP_PROJECT,FSP_ ORG_ID:63959,6282\&cs=1AAA1E35FC40E4C51A7FA46F1191A35FC).

EN ISO 17409 (2020). Electrically Propelled Road Vehicles - Conductive Power Transfer - Safety Requirements. Available at: https://standards.cen.eu/ dyn/www/f?p=204:110:0.FSP_PROJECT,FSP_ORG_ID:69448,6282\&cs= 105701A9A01DAC1D3974D42DA76B705D7).

EN ISO 17427-1 (2018). Intelligent Transport Systems - Cooperative ITS - Part 1: Roles and Responsibilities in the Context of Co-operative ITS Architecture(s). Available at: https://standards.cen.eu/dyn/www/f?p=204:110:0 (FSP_PROJECT,FSP_ ORG_ID:60137,6259\&cs=1D8CDC93EFE27562D6AAA4E09FD0C51AA).

EN ISO 18541-6 (2018). Road Vehicles - Standardized Access to Automotive Repair and Maintenance Information (RMI) - Part 6: L-Category Vehicle Specific RMI Use Cases and Requirements. Available at: https://www.iso. org/standard/67459.html.

EN ISO 19363 (2021). Electrically Propelled Road Vehicles - Magnetic Field Wireless Power Transfer - Safety and Interoperability Requirements. Available at: https:// standards.cen.eu/dyn/www/f?p=204:110:0 (FSP_PROJECT,FSP_ORG_ID: 72427,6282\&cs=10B9FCE557CCE87B4F980E1D61A553F22).

EN ISO 24534 (2010-2019). Automatic Vehicle and Equipment Identification Electronic Registration Identification (ERI) for Vehicles. Available at: https:// standards.cen.eu/dyn/www/f?p=204:110:0 (FSP_PROJECT,FSP_ORG_ID: 31238,6259\&cs=1FA31C4DCBCAF05CE351F3188EB0B7E59).

EN ISO 24978 (2009). Intelligent Transport Systems - ITS Safety and Emergency Messages Using Any Available Wireless media - Data Registry Procedures. Available at: https://standards.cen.eu/dyn/www/f?p=204:110:0 (FSP_PROJECT,FSP_ORG_ ID:27136,6259\&cs=14D7DA3A29814CBD765E2F8E6C277DE66).

European Commission (2020). Circular Economy Action Plan - for a Cleaner and More Competitive Europe. Available at: https://ec.europa.eu/jrc/communities/ en/community/city-science-initiative/document/circular-economy-action-plancleaner-and-more-competitive. 
European Commission (2019b). EU Road Safety Policy Framework 2021-2030 Next Steps towards Vision Zero. Available at: https://ec.europa.eu/transport/ sites/transport/files/legislation/swd20190283-roadsafety-vision-zero.pdf.

European Commission (2019a). The European Green Deal. Available at: https://ec. europa.eu/info/sites/info/files/european-green-deal-communication_en.pdf.

European Union (2019). Regulation (EU) 2019/2144 of the European Parliament and of the Council. Available at: https://eur-lex.europa.eu/eli/reg/2019/2144/oj.

Filippi, A., Moerman, K., Martinez, V., Turley, A., Haran, O., and Toledano, R. (2018). IEEE802.11p Ahead of LTE-V2v for Safety Applications". Whitepaper. Available at: https://www.nxp.com/docs/en/white-paper/LTE-V2V-WP.pdf.

Frost Sullivan (2017). Vehicle-to-Everything Technologies for Connected Cars DSRC and Cellular Technologies Drive Opportunities. Available at: https:// www.researchandmarkets.com/reports/4391696/vehicle-to-everything-technologiesfor-connected.

Gpp3 (2018). TS 22.261 v16.5.0 - 3rd Generation Partnership Project; Technical Specification Group Services and System Aspects; Service Requirements for the 5G System; Stage 1 (Release 16). 5G and 3GPP September 2018. Available at: http://www.3gpp.org/ftp/specs/archive/22_series/22.261/22261-g50.zip

GPP3 (2019). TS 33.501, Security Architecture and Procedures for 5G System.

Gu, H., and Moerman, K. (2020). Choosing the Right V2X Technology. Straight Talk on DSRC and 5G. Available at: https://www.nxp.com/docs/en/supportinginformation/CHOOSING-RIGHT-V2X-TECHNOLOGY.pdf.

Gupta, A., Anpalagan, A., Guan, L., and Khwaja, A. S. (2021). Deep Learning for Object Detection and Scene Perception in Self-Driving Cars: Survey, Challenges, and Open Issues. Array 10, 100057. doi:10.1016/j.array.2021.100057

Han, Y., Wang, X., Leung, V. C. M., Niyato, D., Yan, X., and Chen, V. (2019). Convergence of Edge Computing and Deep Learning: a Comprehensive Survey. arXiv preprints arxiv:1907.08349v1

Hashim, A., Saini, T., Bhardwaj, H., Jothi, A., and Vinay Kumar, A. (2019). "Application of Swarm Intelligence in Autonomous Cars for Obstacle Avoidance," in Integrated Intelligent Computing, Communication and Security. Studies in Computational Intelligence. Editors A. Krishna, K. Srikantaiah, and C. Naveena (Singapore: Springer), Vol. 771, 393-404. doi:10.1007/978-981-10-8797-4_41

He, Y., Zhao, N., and Yin, H. (2018). Integrated Networking, Caching, and Computing for Connected Vehicles: a Deep Reinforcement Learning Approach. IEEE Trans. Veh. Technol. 67, 44-55. doi:10.1109/TVT.2017.2760281

Heidari, H., Rassõlkin, A., Kallaste, A., Vaimann, T., Andriushchenko, E., Belahcen, A., et al. (2021). A Review of Synchronous Reluctance MotorDrive Advancements. Sustainability 13 (2), 729. doi:10.3390/ su13020729

IATF 16949 (2016). Technical Specification - Quality Management Systems for Automotive Production and Relevant Service Parts Organizations. Available at: https://www.iatfglobaloversight.org/iatf-169492016/about/.

IEC 61508 (2010). Functional Safety of Electrical/electronic/programmable Electronic Safety-Related Systems. Available at: https://webstore.iec.ch/ publication $/ 5515$.

IEC 61851-1 (2017). Electric Vehicle Conductive Charging System - Part 1: General Requirements. Available at: https://webstore.iec.ch/publication/33644.

IEC 61851-23 (2014). Electric Vehicle Conductive Charging System - Part 23: DC Electric Vehicle Charging Station. Available at: https://webstore.iec.ch/ publication/6032.

IEC 61851-24 (2014). Electric Vehicle Conductive Charging System - Part 24: Digital Communication between a d.C. EV Charging Station and an Electric Vehicle for Control of d.C. Charging. Available at: https://webstore.iec.ch/ publication/6033.

IEC 62752 (2016). In-cable Control and protection Device for Mode 2 Charging of Electric Road Vehicles (IC-CPD). Available at: https://webstore.iec.ch/ publication/24284.

IEEE 1609 Parts (2006-2020). Standards for Wireless Access in Vehicular Environments (WAVE). Available at: https://standards.ieee.org/standard/ 1609_0-2019.html.

IEEE $802.11 \mathrm{p}$ (2010). IEEE Standard for Information Technology - Local and Metropolitan Area Networks - Specific Requirements - Part 11: Wireless LAN Medium Access Control (MAC) and Physical Layer (PHY) Specifications Amendment 6: Wireless Access in Vehicular Environments. Available at: https://standards.ieee.org/standard/802_11p-2010.html.

IEEE P1228 (2019). Standard for Automotive System Image Quality. Available at: https://standards.ieee.org/project/2020.html.
IEEE P2020 (2016). Standard for Automotive System Image Quality. Available at: https://standards.ieee.org/project/2020.html.

IEEE P2846 (2020). Assumptions for Models in Safety-Related Automated Vehicle Behavior. Available at: https://standards.ieee.org/project/2846.html.

IEEE P2851 (2019). Standard for Exchange/Interoperability Format for Functional Safety Analysis and Functional Safety Verification of IP, SoC and Mixed Signal ICs. Available at: https://standards.ieee.org/project/2851.html.

IEEE P802.11bd (2020). IEEE Standard for Information Technology Telecommunications and Information Exchange between Systems Local and Metropolitan Area Networks - Specific Requirements - Part 11: Wireless LAN Medium Access Control (MAC) and Physical Layer (PHY) Specifications Amendment: Enhancements for Next Generation V2X. Available at: https:// standards.ieee.org/project/802_11bd.html.

IPC 2551 (2020). International Standard for Digital Twins. Available at: https:// shop.ipc.org/IPC-2551-English-D.

ISO 15623 (2013). Intelligent Transport System - Forward Vehicle Collision Systems - Performance Requirements and Test Procedures. Available at: https://www.iso.org/standard/56655.html.

ISO 19206-2 (2018). Road Vehicles - Test Devices for Target Vehicles, Vulnerable Road Users and Other Objects, for Assessment of Active Safety Functions - Part 2: Requirements for Pedestrian Targets. Available at: https://www.iso.org/ standard/63992.html.

ISO 19206-3 (2021). Road Vehicles - Test Devices for Target Vehicles, Vulnerable Road Users and Other Objects, for Assessment of Active Safety Functions - Part 3: Requirements for Passenger Vehicle 3D Targets. Available at: https://www. iso.org/standard/70133.html.

ISO 19206-4 (2020). Road Vehicles - Test Devices for Target Vehicles, Vulnerable Road Users and Other Objects, for Assessment of Active Safety Functions - Part 4: Requirements for Bicyclist Targets. Available at: https://www.iso.org/ standard/70134.html.

ISO 19237 (2017). Intelligent Transport Systems - Bicyclist Detection and Collision Mitigation Systems (BDCMS) - Performance Requirements and Test Procedures. Available at: https://www.iso.org/standard/64111.html.

ISO 22078 (2020). Intelligent Transport Systems - Bicyclist Detection and Collision Mitigation Systems (BDCMS) - Performance Requirements and Test Procedures. Available at: https://www.iso.org/standard/72508.html.

ISO 26262 Parts 1-12 (2018). Road Vehicles - Functional Safety. Available at: https://webstore.ansi.org/Standards/ISO/ISO26262RoadVehiclesFunctional? source $=$ blog.

ISO 26262 (2018). Road Vehicles - Functional Safety. Available at: https://www.iso. org/standard/68383.html.

ISO 26262 (2019). Wikipedia. Available at: https://en.wikipedia.org/wiki/ISO_ 26262ISO/PAS 21448.

ISO 26262-3 (2018). Road Vehicles - Functional Safety - Part 3: Concept Phase. International Standardization Organization. Availableat: https://www.iso.org/ obp/ui/\#iso:std:iso:26262:-3:en.

ISO 8608 (2016). Mechanical Vibration - Road Surface Profiles - Reporting of Measured Data. Available at: https://www.iso.org/standard/71202.html.

ISO 8855 (2011). Road Vehicles - Vehicle Dynamics and Road-Holding Ability Vocabulary. Available at: https://www.iso.org/standard/51180.html.

ISO SAE 22736 (2020). Intelligent Transport Systems. Taxonomy and Definitions for Terms Related to Driving Automation Systems for On-Road Motor Vehicles. Available at: https://www.iso.org/standard/73766.html.

ISO SAE FDIS 21434 (2020). Road Vehicles - Cybersecurity Engineering. Available at: https://www.iso.org/standard/70918.htmlhttps://www.sae.org/standards/ content/iso_sae21434.d1/.

ISO TR 4804 (2020). Road Vehicles - Safety and Cybersecurity for Automated Driving Systems - Design, Verification, and Validation. Available at: https:// www.iso.org/standard/80363.html.

ISO/IEC 25010 (2011). Systems and Software Engineering - Systems and Software Quality Requirements and Evaluation (SQuaRE) - System and Software Quality Models. Available at: https://www.iso.org/standard/35733.html.

ISO/IEC 27001 (2013). Information Technology - Security Techniques - Information Security Management Systems - Requirements. Available at: https://www.iso.org/isoiec-27001-information-security.html.

ISO/IEC AWI 24029-2 (2021). Artificial Intelligence (AI) - Assessment of the Robustness of Neural Networks - Part 2: Methodology for the Use of Formal Methods. Available at: https://www.iso.org/standard/79804.html. 
ISO/IEC TR 24029-1, (2021). Artificial Intelligence (AI) - Assessment of the Robustness of Neural Networks - Part 1: Overview. Available at: https://www. iso.org/standard/77609.html

ISO/PAS 21448 (2019). Road Vehicles - Safety of the Intended Functionality. Available at: https://www.iso.org/standard/70939.html.

ISO/PRF 22737 (2021). Intelligent Transport Systems - Low-Speed Automated Driving (LSAD) Systems for Predefined Routes - Performance Requirements, System Requirements and Performance Test Procedures. Available at: https:// www.iso.org/standard/73767.html.

Kim, K., Kim, J. S., Jeong, S., Park, J.-H., and Kim, H. K. (2021). Cybersecurity for Autonomous Vehicles: Review of Attacks and Defense. Comput. Security 103, 102150. doi:10.1016/j.cose.2020.102150

Koopman, P., and Wagner, M. (2016). Challenges in Autonomous Vehicle Testing and Validation. SAE Int. J. Trans. Saf. 4 (1), 15-24. doi:10.4271/2016-01-0128

Li, L., Hao, R., Ma, W., Qi, X., and Diao, C. (2018a). Swarm Intelligence Based Algorithm for Management of Autonomous Vehicles on Arterials. SAE Technical Paper 2018-01-1646. doi:10.4271/2018-01-1646

Li, Z., Gavrilyuk, K., Gavves, E., Gavrilyuk, K., Gavves, E., Jain, M., et al. (2018b). VideoLSTM Convolves, Attends and Flows for Action Recognition. Computer Vis. Image Understanding 166, 41-50. doi:10.1016/j.cviu.2017.10.011

Lüdtke, A. (2014). Cognitive Architecture for Safety Critical Task Simulation. Available at: https://hcd.offis.de/wordpress/wp-content/uploads/2015/05/ Part1-Introduction-to-CASCaS.pdf.

Lüdtke, A., Weber, L., Osterloh, J.-P., and Wortelen, B. (2009). "Modeling Pilot and Driver Behavior for Human Error Simulation," in Digital Human Modeling. ICDHM 2009. Lecture Notes in Computer Science. Editor V. G. Duffy (Berlin, Heidelberg: Springer), Vol. 5620, 403-412. doi:10.1007/978-3-642-02809-0_43

Meletis, P., and Dubbelman, G. (2018). "Training of Convolutional Networks on Multiple Heterogeneous Datasets for Street Scene Semantic Segmentation," in 2018 IEEE Intelligent Vehicles Symposium (IV), Jun. 2018, pp. 1045-1050. doi:10.1109/IVS.2018.8500398

MobilEye (2019). Implementing the RSS Model on NHTSA Pre-crash Scenarios. Available at: https://static.mobileye.com/website/corporate/rss/rss_on_ nhtsa.pdf.

Moerman, K., Martinez, V., Van Doorn, J., and Daalderop, G. (2020). Comparison of PHY and MAC Properties of LTE-V Mode 4 and IEEE802.11p, Effects on Coexistence, Possible Mitigating Measures and Input to Standardization. Internal CONCORDA project delivery version 2.5 .

Mohseni, S., Pitale, M., Singh, V., and Wang, Z. (2020). Practical Solutions for Machine Learning Safety in Autonomous Vehicles. Accepted at AAAI's Workshop on Artificial Intelligence Safety (Safe AI) 2020. Available at: https://arxiv.org/abs/1912.09630v1.

Molina-Masegosa, R., Gozalvez, J., and Sepulcre, M. (2020). Comparison of IEEE 802.11p and LTE-V2x: An Evaluation with Periodic and Aperiodic Messages of Constant and Variable Size. IEEE Access 8, 121526-121548. doi:10.1109/ ACCESS.2020.3007115

Munasinghe, G. K., and Murtaza, M. (2020). Analyzing Vehicle-To-Everything Communication for Intelligent Transportation System: Journey from IEEE 802.11p to 5G and Finally towards 6G. 2020 5th International Conference on Innovative Technologies in Intelligent Systems and Industrial Applications. Sydney, Australia. CITISIA), 1-7. doi:10.1109/CITISIA50690.2020.9371804

Naik, G., Choudhury, B., Choudhury, B., and Park, J.-M. (2019). IEEE 802.11bd \& 5G NR V2X: Evolution of Radio Access Technologies for V2X Communications. IEEE Access 7, 70169-70184. doi:10.1109/ACCESS.2019.2919489

Nascimento, A. M., Vismari, L. F., Molina, C. B. S. T., Cugnasca, P. S., Camargo, J. B., Almeida, J. R. d., et al. (2020). A Systematic Literature Review about the Impact of Artificial Intelligence on Autonomous Vehicle Safety. IEEE Trans. Intell. Transport. Syst. 21 (12), 4928-4946. doi:10.1109/TITS.2019.2949915

OADF (2021). Open AutoDrive Forum. Available at: https://www. openautodrive.org/.

OAA (2014). Open Automotive Alliance. Available at: https://www. openautoalliance.net/.

Ottella, M., Perlo, P., Vermesan, O., John, R., Gehrels, K., Gall, H., et al. (2010). "Embedded Systems: The Migration from ICE Vehicles to Electric Vehicles," in Advanced Microsystems for Automotive Applications 2010. Editors G. Meyer and J. Valldorf (Berlin, Heidelberg: VDI Buch. Springer), 3-12. doi:10.1007/978-3-642-16362-3_1
Pohl, J. (2015). Artificial Superintelligence: Extinction or Nirvana? InterSymp-2015. 27th International Conference on Systems Research, Informatics and Cybernetics (IIAS). Baden-Baden, Germany3 August, 2015. Available at: https://www. researchgate.net/publication/281748315_Artificial_Superintelligence_ Extinction_or_Nirvana.

Pype, P., Daalderop, G., Schulz-Kamm, E., Walters, E., Blom, G., and Westermann, S. (2017b). "Intelligent Transport Systems: The Trials Making Smart Mobility a Reality," in Intelligent Transport Systems: The Trials Making Smart Mobility a Reality. Automated Driving. Editors D. Watzenig and M. Horn (Cham: Springer), 595-600. doi:10.1007/978-3-319-31895-0_30

Pype, P., Daalderop, G., Schulz-Kamm, E., Walters, E., and von Grafenstein, M. (2017a). "Privacy and Security in Autonomous Vehicles," in Privacy and Security in Autonomous Vehicles. Automated Driving. Editors D. Watzenig and M. Horn (Cham: Springer), 17-27. doi:10.1007/978-3-319-31895-0_2

Rigas, E. S., Ramchurn, S. D., and Ramchurn, N. (2015). Managing Electric Vehicles in the Smart Grid Using Artificial Intelligence: A Survey. IEEE Trans. Intell. Transport. Syst. 4, 1619-1635. doi:10.1109/TITS.2014.2376873

SAE International (2019). SAE J3016 ${ }^{\mathrm{TM}}$ Levels of Driving Automation. Available at: https://www.sae.org/news/2019/01/sae-updates-j3016-automated-drivinggraphic\#: :text=The\%20J3016\%20standard\%20defines $\% 20$ six,\%2Dvehicle\% 20 (AV)\%20capabilities.\&text=The\%20latest $\% 20$ J3016\%20graphic\%20is\% 20a\%20living\%20document.,-It\%20will\%20continue.

SAE J1772 (2017). SEA Electric Vehicle and Plug in Hybrid Electric Vehicle Conductive Charge Coupler. Available at: https://www.sae.org/standards/ content/j1772_201710/.

SAE J2735 (2007). - V2X Communication Message Set Dictionary. Available at: https://www.sae.org/standards/content/j2735_202007./

SAE J2954 (2020). Wireless Power Transfer for Light-Duty Plug-In/Electric Vehicles and Alignment Methodology. Available at: https://www.sae.org/ standards/content/j2954_202010/.

SAE J3016 (2018). Taxonomy and Definitions for Terms Related to Driving Automation Systems for On-Road Motor Vehicles. Available at: https:// www.sae.org/standards/content/j3016_201806/.

SAE J3061 (2016). Cybersecurity Guidebook for Cyber-Physical Vehicle Systems. Available at: https://www.sae.org/standards/content/j3061_201601/.

Searle, J. R. (1998). Mind, Language and Society: Philosophy in the Real World. New York, NY: Basic Books. 978-0-465-04521-1.

Selvanesan, S., Thomas, R. R., Thomas, R. R., Fehrenbach, T., Wirth, T., and Hellge, C. (2018). "Towards Advanced V2X Multimedia Services for 5G Networks," in Towards Advanced V2X Multimedia Services for 5G Networks, "2018 IEEE International Symposium on Broadband Multimedia Systems and Broadcasting. Valencia, Spain. BMSB), 1-6. doi:10.1109/BMSB.2018.8436925

Shalev-Shwartz, S., Shammanh, S., and Shashua, A. (2017). On a Formal Model of Safe and Scalable Self-Driving Cars. Available at: https://arxiv.org/pdf/1708.06374.pdf.

Shimizu, T., Lu, H., Kenney, J., and Nakamura, S. (2019). "Comparison of DSRC and LTE-V2x PC5 Mode 4 Performance in High Vehicle Density Scenarios" in Proc. ITS World Congr., Singapore, Oct. 2019, 1-7.

Song, H. M., Woo, J., and Kim, H. K. (2020). In-vehicle Network Intrusion Detection Using Deep Convolutional Neural Network. Vehicular Commun. 21, 100198. doi:10.1016/j.vehcom.2019.100198

Storck, C. R., and Duarte-Figueiredo, F. (2020). A Survey of 5G Technology Evolution, Standards, and Infrastructure Associated with Vehicle-ToEverything Communications by Internet of Vehicles. IEEE Access 8, 117593-117614. doi:10.1109/ACCESS.2020.3004779

Sun, D., Ou, Q., Yao, X., Gao, S., Wang, Z., Ma, W., et al. (2020). "Integrated Human-Machine Intelligence for EV Charging Prediction in 5G Smart Grid". J. Wireless Com Netw., 2020, 139. doi:10.1186/s13638-020-01752-y

Tang, F., Kawamoto, Y., Kato, N., Kawamoto, Y., Kato, N., and Liu, J. (2020). Future Intelligent and Secure Vehicular Network toward 6G: Machine-Learning Approaches. Proc. IEEE 108 (2), 292-307. doi:10.1109/JPROC.2019.2954595

Thomas, J. (2016). In Defense of Philosophy: a Review of Nick Bostrom, Superintelligence: Paths, Dangers, Strategies. J. Exp. Theor. Artif. Intelligence 28 (6), 1089-1094. doi:10.1080/0952813X.2015.1055829

United Nations (2015b). The 17 Goals. Available at: https://sdgs.un.org/goals.

United Nations (2015a). Transforming Our World: The 2030 Agenda for Sustainable Development. Available at: https://sustainabledevelopment.un.org/content/ 
documents/21252030\%20Agenda\%20for\%20Sustainable\%20Development\% 20web.pdf.

Usman, A., Song, H., Bilal, A., Alazab, M., and Jolfaei, A. (2020). Securing Smart Vehicles from Relay Attacks Using Machine Learning. J. Supercomputing 76 (4), 2665-2682. doi:10.1007/s11227-019-03049-4

Vermesan, O. (2020b). AI for Digitizing Industry Road-Mapping Requirements. AI4DI ECSEL H2020 Project.

Vermesan, O., Bahr, R., Brevil, D., Bosi, I., Falcitelli, M., Velizhev, A., et al. (2020a). "IoT Technologies for Connected and Automated Driving Applications," in Internet of Things - the Call of the Edge - Everything Intelligent Everywhere. Editors O. Vermesan and J. Bacquet (Gistrup: River Publishers), 255-306. doi:10.13052/rp-9788770221955 Available at: https://www.riverpublishers. com/pdf/ebook/chapter/RP_9788770221955C6.pdf.

Vermesan, O., Bahr, R., John, R., Ottella, M., Gjølstad, R., Buckholm, O., et al. (2021a). "Advancing the Design of Fail-Operational Architectures, Communication Modules, Electronic Components, and Systems for Future Autonomous/ Automated Vehicles," in Intelligent System Solutions for Auto Mobility and beyond. AMAA 2020. Lecture Notes in Mobility. Editors C. Zachäus and G. Meyer (Cham: Springer), 53-71. doi:10.1007/978-3-030-65871-7_5

Vermesan, O., Blystad, L.-C., Zafalon, R., Moscatelli, A., Kriegel, K., Mock, R., et al. (2011). "Internet of Energy - Connecting Energy Anywhere Anytime," in Advanced Microsystems for Automotive Applications 2011. Editors G. Meyer and J. Valldorf (Berlin, Heidelberg: VDI Buch. Springer), 33-48. doi:10.1007/978-3-642-21381-6_4 Vermesan, O., John, R., Pype, P., Daalderop, G., Ashwathnarayan, M., Bahr, R., et al. (2021b). "Internet of Vehicles - System of Systems Distributed Intelligence for Mobility Applications," in Intelligent Technologies for Internet of Vehicles. Internet of Things (Technology, Communications and Computing). Editors N. Magaia, G. Mastorakis, C. Mavromoustakis, E. Pallis, and E. K. Markakis (Cham: Springer), 93-147. doi:10.1007/978-3-030-76493-7_4

Vermesan, O., Sans, M., Hank, P., Farrall, G., Packer, J., Cesario, N., et al. (2015). "Advanced Electronic Architecture Design for Next Electric Vehicle
Generation," in Electric Vehicle Systems Architecture and Standardization Needs. Lecture Notes in Mobility. Editors B. Müller and G. Meyer (Cham: Springer), 117-141. doi:10.1007/978-3-319-13656-1_8

Wood, M., Robbel, P., Maass, M., Tebbens, R. D., Meijs, M., Harb, M., et al. (2019). Safety First for Automated Driving. Available at: https://www.daimler.com/ documents/innovation/other/safety-first-for-automated-driving.pdf.

Zou, Y., Luo, Z., and Huang, J.-B. (2018). DF-net: Unsupervised Joint Learning of Depth and Flow Using Cross-Task Consistency. Available at: https:// openaccess.thecvf.com/content_ECCV_2018/html/Yuliang_Zou_DF-Net_ Unsupervised_Joint_ECCV_2018_paper.html (Accessed Feb 02, 2021).

Conflict of Interest: Authors OV and RB were employed by the company SINTEF Digital AS. Author RJ was employed by the company AVL List GmbH. Authors PP and GD were employed by the company NXP Semiconductors. Authors KK and GM were employed by the company Siemens AG. Authors VL, SB and SW were employed by the company Fraunhofer IISB. HES was employed by the company NxTech AS.

Publisher's Note: All claims expressed in this article are solely those of the authors and do not necessarily represent those of their affiliated organizations, or those of the publisher, the editors and the reviewers. Any product that may be evaluated in this article, or claim that may be made by its manufacturer, is not guaranteed or endorsed by the publisher.

Copyright $\odot 2021$ Vermesan, John, Pype, Daalderop, Kriegel, Mitic, Lorentz, Bahr, Sand, Bockrath and Waldhör. This is an open-access article distributed under the terms of the Creative Commons Attribution License (CC BY). The use, distribution or reproduction in other forums is permitted, provided the original author(s) and the copyright owner(s) are credited and that the original publication in this journal is cited, in accordance with accepted academic practice. No use, distribution or reproduction is permitted which does not comply with these terms. 